\title{
A BAYESIAN HIERARCHICAL MODEL FOR MULTIPLE COMPARISONS IN MIXED MODELS
}

\author{
Qie Li
}

\author{
A Dissertation \\ Submitted to the Graduate College of \\ Bowling Green State University in partial fulfillment of \\ the requirements for the degree of \\ DOCTOR OF PHILOSOPHY
}

August 2012

Committee:

Junfeng Shang, Advisor

Gary Oates,

Graduate Faculty Representative

Hanfeng Chen

Wei Ning 


\section{ABSTRACT}

Junfeng Shang, Advisor

Multiple comparison techniques are to compare more than one pair of treatment means by employing various effective methodologies. In some applications, researchers may believe that before the data are collected, the underlying parameters satisfy an order restriction. But some researchers believe otherwise for certain applications. For instance, in quality control experiments, researchers study the effect of different setting of significant factors, such as the temperature, the pressure, and the different types of machines. The researchers believe that in order to find complete significant results, all treatments should be mixed up and comparisons should be applied to each pair of them. To facilitate multiple comparisons, certain methodologies are proposed and applied. Generally, the frequentist and the Bayesian methodologies are used to conduct multiple comparisons.

In this dissertation, we are interested in the Bayesian approach. We propose a hierarchical model in developing and applying multiple comparisons without any restriction in mixed models. The model facilitates inferences in parameterizing the successive differences of the population means, and for them we choose independent prior distributions that are mixtures of a normal distribution and a discrete distribution with its entire mass at zero. For the other parameters, we choose conjugate or non-informative priors, and we derive the full conditional posterior distributions for the parameters in the mixed models. A simulation study is performed to investigate the effectiveness of the proposed hierarchical model. In the simulations, a sequence of different simulated data sets is utilized. To 
determine the optimal priors for the parameters or hyper-parameters, we do comparisons of the different priors for the purpose of making a decision. In the applications, two real data sets are analyzed to compare the population means for illustrating the performance of the proposed in the hierarchical model. The Type I error, Family-wise Error rate (FWER), and the test power are also studied in the simulations. The simulation results exhibit that the proposed hierarchical model can effectively do multiple comparisons while keeping Type I error relative low and the test power reasonable large in our simulation sets.

Gibbs sampler, a special procedure of Markov chain Monte Carlo (MCMC), is used in the simulations to compute the parameters estimates and the posterior probabilities that two population means are equal. The iteration procedure allows one both to determine if any two means are significantly different and to test the homogeneity of all of the means. Hypothesis testing procedure is introduced and the simulations are based on it. The proposed hierarchical model is applied in two real data sets for illustrating the performance of the model. The computing procedure of the proposed hierarchical model can effectively unify parameters estimation, tests of hypotheses, and multiple comparisons in one setting. 


\section{ACKNOWLEDGEMENTS}

First, I would like to thank my dissertation advisor, Dr. Junfeng Shang, for her sincere support and kindness guidance throughout the whole process of my dissertation. I would also like to thank Dr. Hanfeng Chen and Dr. Wei Ning for their advises and suggestions during the process of my dissertation. I would like to especially thank Dr. Gary Oates from the Department of Sociology for taking the precious time out of his busy schedule serving as graduate faculty representative in my dissertation committee.

I am also grateful to the Department of Mathematics and Statistics for all the support and for providing a wonderful research environment. Marcia Seubert and Mary Busdeker have provided their great assistance for me in these years. I greatly appreciate their help.

Finally, I would like to give my deepest gratitude to my parents, my wife Xiangyi Li, and my four-month-old son Aaron for their support and love.

Qie Li

Bowling Green, Ohio, USA

August 2012 


\section{TABLE OF CONTENTS}

1 INTRODUCTION 1

1.1 Objective of Dissertation . . . . . . . . . . . . . . . . . 1

1.2 Outline of Dissertation . . . . . . . . . . . . . . . . . 3

2 BAYESIAN HIERARCHICAL MODELS AND COMPUTATIONAL METHODOLOGIES $\quad 6$

2.1 Introduction . . . . . . . . . . . . . . . . . . . . 6

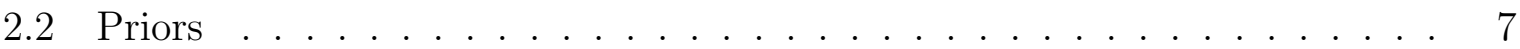

2.3 Posterior Distributions and Full Conditional Posterior Distributions . . . . 9

2.4 Approaches to Simulating from the Posterior Distribution . . . . . . . . . . 12

2.4.1 Metropolis-Hastings Algorithm . . . . . . . . . . . . . . 13

2.4.2 Gibbs Sampler . . . . . . . . . . . . . . . . . 15

2.4 .3 Other Methods . . . . . . . . . . . . . . . . 16

3 REVIEW OF RELEVANT LITERATURE FOR MULTIPLE COM$\begin{array}{ll}\text { PARISONS } & 18\end{array}$

3.1 Introduction . . . . . . . . . . . . . . . . . . . 18

3.2 Frequentist Methods . . . . . . . . . . . . . . . . . . 20

3.2.1 Simultaneous Statistical Inference . . . . . . . . . . . . . . 21

3.2.2 Multiple Comparisons with a Control (MCC) . . . . . . . . . . 22

3.2.3 All-pairwise Comparisons (MCA) . . . . . . . . . . . . 25

3.3 Error Rate . . . . . . . . . . . . . . . . . . . . . . . 28 
3.4 Family-wise Error Rate (FWER) . . . . . . . . . . . . . . . . . . . 29

3.5 Bayesian Methods . . . . . . . . . . . . . . . . . 30

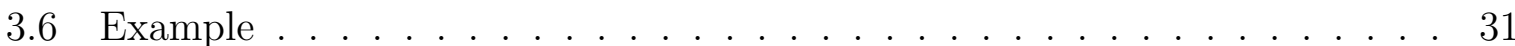

4 DERIVATION OF THE BAYESIAN HIERARCHICAL MODEL FOR MULTIPLE COMPARISONS IN MIXED MODELS 34

4.1 Introduction . . . . . . . . . . . . . . . . . . . . . 34

4.2 The Mixed Model . . . . . . . . . . . . . . . . . . . . . . . . . 35

4.3 Joint Density Function . . . . . . . . . . . . . . . . . . . 36

4.4 Priors and Hyper-priors . . . . . . . . . . . . . . . . . . . . . . . 41

4.5 Full Conditional Distributions . . . . . . . . . . . . . . . . . . 43

4.5.1 Full Conditional Posterior Distribution of $\mu_{1}$. . . . . . . . . . . 43

4.5.2 Full Conditional Posterior Distribution of $\sigma^{2}$ and $\tau^{2} \ldots \ldots$. . . . 46

4.5.3 Full Conditional Posterior Distribution of $\delta_{i} \ldots \ldots$. . . . . . . . 48

4.5.4 Full Conditional Posterior Distribution of $P_{r}$. . . . . . . . . . . . . 53

4.5.5 Full Conditional Posterior Distribution of $\sigma_{\Delta}^{2} \ldots \ldots . . . . . . \quad 55$

4.6 Gibbs Sampling Steps . . . . . . . . . . . . . . . . . 56

5 PRESENTATION OF SIMULATIONS AND APPLICATIONS 58

5.1 Introduction . . . . . . . . . . . . . . . . . . 58

5.2 Hypothesis Testings via Posterior Probability . . . . . . . . . . . . 60

5.3 Presentation of Results for Simulated Data . . . . . . . . . . . . . . . . 64

5.3.1 Simulation 1: Optimal priors for $\sigma_{\Delta}^{2} \ldots \ldots \ldots$. . . . . 64

5.3.2 Simulation 2: Optimal Priors for $\mu_{1} \ldots \ldots \ldots$. . . . . . 78

5.3.3 Simulation 3: Parameter Estimates and Posterior Probabilities . . . 83

5.4 Simulation 4: Familywise Error Rates and Tests Powers . . . . . . . . . . . 90

5.5 Applications for the Proposed Bayesian Hierarchical Model . . . . . . . . . 93

5.5.1 Application on Lead Level Data . . . . . . . . . . . . . . . . . . 93

5.5.2 Application on Midterm Exam Data . . . . . . . . . . . . . . . 99 
6 CONCLUSIONS AND FURTHER DIRECTIONS 103

$\begin{array}{ll}\text { REFERENCES } & 105\end{array}$

APPENDICES: R PROGRAMS 109 


\section{LIST OF TABLES}

3.1 Type I and II Errors in Multiple Hypotheses Testing . . . . . . . . . . . 28

3.2 -value Comparison in Bayesian and Frequentist Method for Height Exam-

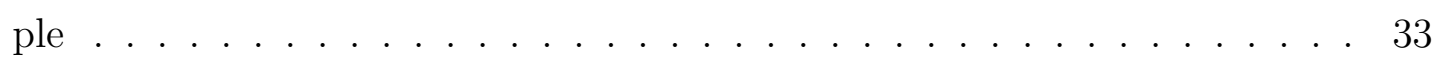

5.1 Simulation Results for Optimal Priors of $\sigma_{\Delta}^{2}$ under Equal Population Means and Sample Size $m=10 \operatorname{Part}(\mathrm{a}) \ldots \ldots \ldots \ldots$

5.2 Simulation Results for Optimal Priors of $\sigma_{\Delta}^{2}$ under Equal Population Means and Sample Size $m=10 \operatorname{Part}(\mathrm{b}) \ldots \ldots \ldots \ldots$. . . . . . . . 67

5.3 Simulation Results for Optimal Priors of $\sigma_{\Delta}^{2}$ under Equal Population Means and Sample Size $m=30 \operatorname{Part}(\mathrm{a}) \ldots \ldots \ldots \ldots$. . . . . . . . 68

5.4 Simulation Results for Optimal Priors of $\sigma_{\Delta}^{2}$ under Equal Population Means and Sample Size $m=30 \operatorname{Part}(\mathrm{b}) \ldots \ldots \ldots \ldots \ldots$

5.5 Simulation Results for Optimal Priors of $\sigma_{\Delta}^{2}$ under Equal Population Means and Sample Size $m=100 \operatorname{Part}(\mathrm{a}) \ldots \ldots \ldots \ldots$

5.6 Simulation Results for Optimal Priors of $\sigma_{\Delta}^{2}$ under Equal Population Means and Sample Size $m=100 \operatorname{Part}(\mathrm{b}) \quad \ldots \ldots \ldots \ldots \ldots 71$

5.7 Simulation Results for Optimal Priors of $\sigma_{\Delta}^{2}$ under Unequal Population Means and Sample Size $m=10 \operatorname{Part}(\mathrm{a}) \ldots \ldots \ldots$. . . . . . . 72

5.8 Simulation Results for Optimal Priors of $\sigma_{\Delta}^{2}$ under Unequal Population Means and Sample Size $m=10 \operatorname{Part}(\mathrm{b}) \ldots \ldots \ldots \ldots \ldots$ 
5.9 Simulation Results for Optimal Priors of $\sigma_{\Delta}^{2}$ under Unequal Population

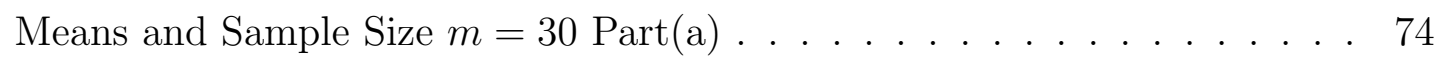

5.10 Simulation Results for Optimal Priors of $\sigma_{\Delta}^{2}$ under Unequal Population Means and Sample Size $m=30$ Part(b) . . . . . . . . . . . . . . 75

5.11 Simulation Results for Optimal Priors of $\sigma_{\Delta}^{2}$ under Unequal Population Means and Sample Size $m=100 \operatorname{Part}(\mathrm{a}) \ldots \ldots$. . . . . . . . 76

5.12 Simulation Results for Optimal Priors of $\sigma_{\Delta}^{2}$ under Unequal Population Means and Sample Size $m=100$ Part(b) . . . . . . . . . . . . . 77

5.13 Simulation Results for Optimal Priors of $\mu_{1}$ under Equal Population Means and Sample Size $m=30 \ldots \ldots$. . . . . . . . . . . . . 79

5.14 Simulation Results for Optimal Priors of $\mu_{1}$ under Equal Population Means and Sample Size $m=100 \ldots \ldots$. . . . . . . . . . . 80

5.15 Simulation Results for Optimal Priors of $\mu_{1}$ under Unequal Population Means and Sample Size $m=30$. . . . . . . . . . . . . . . 81

5.16 Simulation Results for Optimal Priors of $\mu_{1}$ under Unequal Population Means and Sample Size $m=100$. . . . . . . . . . . . . . 82

5.17 Estimation for Parameters and Posterior Probabilities under Equal Population Means and Small Variances . . . . . . . . . . . . . . . . 84

5.18 Estimation for Parameters and Posterior Probabilities under Equal Population Means and Large Variances . . . . . . . . . . . . . . 86

5.19 Estimation for Parameters and Posterior Probabilities under Unequal Population Means and Small Variances . . . . . . . . . . . . . . 88

5.20 Estimation for Parameters and Posterior Probabilities under Unequal Population Means and Large Variances . . . . . . . . . . . . . . . . . 89

5.21 Average Posterior Probability of the Null Hypothesis and Fraction of Times

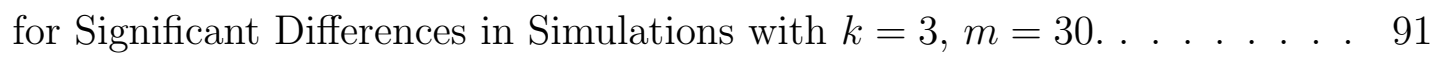

5.22 Estimation Results for Parameters and Posterior Probabilities in Blood Lead Level Application . . . . . . . . . . . . . . . . . . . . . 94 
5.23 Posterior Parameter Estimation Result for Midterm Exam Application . . 100 


\section{LIST OF FIGURES}

5.1 The Posterior Convergence for $\mu_{1}$ in Lead Level Data Set. . . . . . . . . . 96

5.2 The Posterior Convergence for $\sigma^{2}$ in Lead Level Data Set. . . . . . . . . . 96

5.3 The Posterior Convergence for $\sigma_{\tau}^{2}$ in Lead Level Data Set. . . . . . . . . . 96

5.4 The Posterior Distributions for $\delta_{2 \mathrm{~V} 3}, \delta_{2 \mathrm{~V} 4}, \delta_{3 \mathrm{v} 4}$ in Lead Level Data Set. . . 97

5.5 The Posterior Distributions for $\delta_{1 \mathrm{v} 2}, \delta_{1 \mathrm{v} 3}, \delta_{1 \mathrm{v} 4}$ in Lead Level Data Set. . . 98

5.6 The Posterior Convergence for $\mu_{1}$ in Midterm Exam Data Set. . . . . . . . 101

5.7 The Posterior Convergence for $\sigma^{2}$ in Midterm Exam Data Set. . . . . . . . 101

5.8 The Posterior Convergence for $\sigma_{\tau}^{2}$ in Midterm Exam Data Set. . . . . . . . 101

5.9 The Posterior Distributions for $\delta_{1 \mathrm{v} 2}, \delta_{1 \mathrm{v} 3}, \delta_{2 \mathrm{v} 3}$ in Midterm Exam Data Set. 102 


\section{CHAPTER 1}

\section{INTRODUCTION}

\subsection{Objective of Dissertation}

In the last decades, multiple comparisons play an important role in many fields, business,

clinic trials, environmental sciences, and even astrophysics, etc. As in the analysis of variance (ANOVA) comparing more than two treatments (Hsu, 1996) when more than two measurement methods are compared, multiple comparisons provide a simultaneously comparing the extent of agreement between all pairs of methods of concern, and ranking them on the basis of their extent of agreement (Choudharya, P. K. and Yin K., 2010). Before deciding which multiple comparison procedure to use, there are three main components of the problems that need to be identified: 1. the assumptions of the statistical model to use; 2 . the comparison or testing objectives of the study; 3 . the set (family) of elements to be tested. Once these three elements are determined, we can identify an appropriate method of inference.

Selection of a family of inference is considered the most important, and most contro- 
versial, aspect of multiple comparisons. Statistical inferences can vary greatly depending upon how this family is selected, and specifically, upon how many tests or comparisons are included in the family. For example, if we would like a multiple comparison of treatment groups A, B and C, one nature family of comparisons might be all pairs (A vs. B), (B vs. C) and (A vs. C). However, if this objective is to compare the treatments with one standard control, say group C for example, then this family may be safely reduced to just (A vs. C) and (B vs. C). Thus, the inferences can vary dramatically, depending upon the choice of the family of tests. The power of multiple comparisons can improved by selecting the family to be as small as possible, while still addressing all questions of interest.

Statistically, we can use the frequentist and the Bayesian method to conduct multiple comparisons procedures. From frequentist perspectives, a number of procedures have been carried out in the last century. Some of the procedures are commonly used, such as Fisher's method (1925), Tukey's method (1953) and Dunnet's method (1955). In these procedures, $p$-values are used to do multiple comparisons, and Type I error rates and Family-wise Error Rates (FWER) are intended to be controlled as small as possible or to be balanced with test powers. To reach such kind of goal, Bonferrnoi's method (1979) is extensively used.

From Bayesian perspectives, quite a few procedures have been done for multiple comparisons. In the early 1990s, significant developments were made in Bayesian methods for order-restricted inferences. Gelfand, Hills, Racine-Poon, \& Smith (1990) provided Bayesian estimates of order-restricted normal means with arbitrary variances. In life- 
testing models, Molitor \& Sun (2002) considered situations in which means and variances simultaneously satisfy order restrictions and provided Bayesian estimates of the means and variances. Under the order restriction assumption, Shang (2008) proposed a hierarchical model in the setting of mixed models with fixed treatment effect and random effects, however, not all multiple comparisons are under a simple order assumption. For instance, in the quality control experiments or products evaluation process, researchers study the effect of different setting of significant factors, such as the temperature, the pressure, and the different types of machines. In such situations, the researchers may wish to conduct multiple comparisons for all the different pairs.

The objective of this dissertation is to propose a hierarchical model in order to perform all pairwise comparisons in the mixed model without any order restriction. We parameterize the means by using their successive differences of population means. The advantage of this proposed model is that it can combine multiple comparisons, parameters estimations, and the posterior probabilities calculations in one computational procedure. A simulation study and the applications of two real data sets are fulfilled for examining the effectiveness of the proposed model.

\subsection{Outline of Dissertation}

This dissertation is composed of six chapters. Chapter 1 gives an introduction to this dissertation.

Chapter 2 provides several background materials that serve as the foundation of this 
dissertation. The description includes Bayesian idea, introduction of prior, and procedures for developing posterior distribution and the full conditional posterior distribution, along with an overview of Markov chain Monte Carlo (MCMC) approach for simulating from the full conditional posterior distribution. Detailed descriptions of Metropolis-Hastings algorithm and Gibbs sampler method are also presented.

Chapter 3 provides an overall introduction to different types of multiple comparison methods in both frequentist and Bayesian methods. After discussing the simultaneous statistical inference of one-way model, two commonly used multiple comparison inferences, multiple comparisons with a control (MCC) and all-pairwise comparisons (MCA) are introduced. Several well-established methods: Tukey's method (1953) and Dunnet's method (1955) are also described. This chapter also includes a brief introduction of error rate, including Type I, Type II, and Famliy-wise Error Rate (FWER). Finally, the chapter focuses on discussing multiple comparisons in Bayesian methods, and an example shows the different approaches using the frequentist and the Bayesian method on a hypothesis testing problem.

In Chapter 4, a Bayesian hierarchical model for multiple comparisons in mixed models is derived. First the joint density function for the data set is developed and proposed. After providing the prior density functions for both parameters and hyper-parameters, the full conditional posterior distributions are developed. At the end of this chapter, an effectively Gibbs sampler algorithm for the simulation is presented.

In Chapter 5, priors for $\sigma_{\triangle}^{2}$ and $\mu_{1}$ are specified in the Gibbs sampler framework in simulations. After choosing the optimal priors, a simulation study about the param- 
eters estimation and posterior probabilities that any two means are equal for multiple comparisons is conducted. In this study, the data sets are simulated with different population means and samples sizes. Two applications of real data sets (Lead level data set and Midterm Exam data set) are analyzed for investigating the behavior of the proposed model. In the simulations and applications, we discuss the simulation objectives, parameters estimation, and detailed conclusion of the results.

In Chapter 6, the concluding remarks and future work directions are discussed. In the future, we would like to use Bayesian hierarchical models to do multiple comparisons in other mixed models and with missing data involved. 


\section{CHAPTER 2}

\section{BAYESIAN HIERARCHICAL MODELS}

\section{AND COMPUTATIONAL}

\section{METHODOLOGIES}

\section{$2.1 \quad$ Introduction}

Bayesian statistics is based on the theorem first discovered by Reverend Thomas Bayes and published after his death in the paper An Essay Towards Solving a Problem in the Doctrine of Chances by his friend Richard Price in Philosophical Transactions of the Royal Society. Bayes' theorem is very clever restatement of the conditional probability formula. It gives a method for updating the probabilities of unobserved events, given that another related event has occurred. This means that we have a prior probability for the unobserved event, and we update this to get its posterior probability, given the occurrence of the related event, and we can use the posterior distribution to carry out all inferences. In this chapter we introduce literature of Bayesian method and relevant knowledge 
of hierarchical Bayesian methods which we will use in the following sections. In Section 2.2 several different ways to set a prior are discussed. In Section 2.3 we show methods to construct a posterior distribution and derive a full conditional posterior distribution. In Section 2.4 several approaches for computational statistics in hierarchical models are presented and discussed.

\subsection{Priors}

A prior distribution of a parameter is the probability distribution that represents one's uncertainty about the parameter before the current data are examined. We cannot carry out any Bayesian inference or perform any modeling without using a prior distribution. So before taking data, we must have a prior probability distribution that measure how plausible we consider each possible parameter value before looking at the data.

Our prior distribution must be highly subjective, because somebody else can have his/her own prior belief about the unknown values of the parameters. Not everyone agrees with this notion of subjectivity when it comes to specifying prior distributions. There has long been a desire to obtain results that are objectively valid. Within the Bayesian paradigm, this can be somewhat achieved by using prior distributions that are "objective" (that is, that have a minimal impact on the posterior distribution). Such distributions are called objective or noninformative priors. However, while noninformative priors are very popular in some applications, they are not always easy to construct.

Disregarding subjectively or objectively, let $p$ represent the proportion of an event 
in the population, and the value of $p$ is unknown. Albert J. (2006) indicates that in the Bayesian viewpoint a person's beliefs about the uncertainty in this proportion are represented by a probability distribution placed on this parameter, and this distribution reflects the person's subjective prior opinion about plausible values of $p$. Several ways to sign prior for $p$. A simple approach for assessing a prior is to write down a list of plausible proportion values and then assign weights to these values. This method is called a discrete prior. This method is straight forward, easily understood and calculable. However, this prior could be very objective.

Since the proportion is a continuous parameter, an alternative approach is to construct a density $g(p)$ on the interval $(0,1)$ that represents the person's initial beliefs. Actually, this approach is widely used in Bayesian method and also in this dissertation. For example, we illustrate the parameter $P_{r}$ in (4.4), the proportion of $\delta_{i}$ equals to zero, and try to make $P_{r}$ as noninformative as possible. A convenient family of densities for a proportion is beta with kernel proportional to

$$
g\left(P_{r}\right) \propto P_{r}^{\alpha_{r}}\left(1-P_{r}\right)^{\beta_{r}-1}
$$

where the hyper-parameters $a_{r}$ and $b_{r}$ are chosen to reflect the my prior beliefs about $\operatorname{Pr}$, details are discussed in Section 5.1. 


\subsection{Posterior Distributions and Full Conditional Posterior Distributions}

Bayesian theorem combines the two sources of information about the unknown parameter value: the prior density and the observed data Bolstad, W. M. (2010). The prior density gives our relative belief weights of every possible parameter value before we observe the data. The likelihood function of the observed data gives the relative weights to every possible parameter value that comes from the observed data. Bayesian theorem combines these into the posterior density, which gives our relative belief weight of the parameter value after observing the data.

With a set of observation $y=\left(y_{1}, \ldots, y_{n}\right)$, by a collection of unknown parameters of a particular dimension (let's say $d), \theta=\left(\theta_{1}, \ldots, \theta_{d}\right)$, we suppose that $\theta$ is a random quantity sampled from a prior distribution $\pi(\theta \mid \lambda)$, where $\lambda$ is a vector of hyper-parameters. For instance, $y_{i}$ might be the observed abundance of a particular species in areal unit $i$ or it might be the observed frequency of a particular allele type in population $i . \theta_{i}$ would then be true abundance of the species in unit $i$ or the true allele proportion in population $i$. Finally, $\lambda$ is a parameter controlling like spatial similarity across areal units or variation among populations. If $\lambda$ is known, inference concerning $\theta$ is based on it's posterior distribution,

$$
p(\theta \mid y, \lambda)=\frac{p(y, \theta \mid \lambda)}{p(y \mid \lambda)}=\frac{p(y, \theta \mid \lambda)}{\int p(y, \theta \mid \lambda) d \theta}=\frac{f(y \mid \theta) \pi(\theta \mid \lambda)}{\int f(y \mid \theta) \pi(\theta \mid \lambda) d \theta}
$$

Notice the contribution of both the data (in the form of the likelihood $f$ ) and the external 
knowledge or opinion (in the form of prior $\pi$ ) to the posterior distribution. However, in practice, $\lambda$ will not be known, a second stage (or hyper-prior) distribution $h(\lambda)$ will often required, then the posterior distribution above will be replaced with

$$
p(\theta \mid y)=\frac{p(y, \theta)}{p(y)}=\frac{\int f(y \mid \theta) \pi(\theta \mid \lambda) h(\lambda) d \lambda}{\int f(y \mid \theta) \pi(\theta \mid \lambda) h(\lambda) d \theta d \lambda} .
$$

Clard, J. S. and Gelfand, A. E. (2006) indicated that Bayesian inferential paradigm offers potentially attractive advantage over the classical frequentist statistical approach through its more philosophically sound foundation, its unified approach to data analysis, and its ability to formally incorporating prior opinion or external empirical evidence into the results via the prior distribution $\pi$. The Bayesian approach better captures uncertainty (both with regard to parameters in models and with regard to model specification itself). It also provides exact inference, avoiding asymptotic whose adequacy may be difficult to assess. Scientists, formerly reluctant to adopt the Bayesian approach due to its subjectivity and a lack of necessary computational tools, are now turning to it with increasing regularity as classical inadequate.

However, if the parameter space is high dimensional, finding the posterior using Bayesian theorem is easy in theory, but integral given above is very difficult to evaluate. In order to find an inexpensive, high-speed computing equipment and software, enabling the application of recently developed Markov chain Monte Carlo (MCMC) integration method, such as the Metropolis-Hastings algorithm (Metropolis et al., 1953; Hastings, 1970) and the Gibbs sampler (Geman \& Geman, 1984; Gelfand \& Smith, 1990). In this 
dissertation, we will use Gibbs sampling method for parameter estimation and hypothesis testing.

There is one thing about Gibbs sampler method, and it requires to decompose the joint posterior distribution into the full conditional posterior distribution for each parameter in the model and then sample from them. The sampler can be efficient when the parameters are not highly dependent on each other and the full conditional distributions are easy to sample from. Some researchers favor this algorithm because it does not require an instrumental proposal distribution as Metropolis methods do. However, while deriving the conditional distributions can be relatively easy, it is not always possible to find an efficient way to sample from these conditional distributions.

Suppose $\theta=\left(\theta_{1}, \ldots \theta_{d}\right)^{\prime}$ is the parameter vector, $p(y \mid \theta)$ is the likelihood, and $\pi(\theta)$ is the prior distribution. Then the full conditional posterior distribution of $\pi\left(\theta_{i} \mid \theta_{j}, i \neq j, y\right)$ is proportional to the joint posterior density; that is,

$$
\pi\left(\theta_{i} \mid \theta_{j}, i \neq j, y\right) \propto p(y \mid \theta) \cdot \pi(\theta)
$$

For instance, the one-dimensional conditional distribution of $\theta_{1}$ given $\theta_{i}=\theta_{j}^{\star}, 2 \leq j \leq d$, is computed as the following:

$$
\pi\left(\theta_{i} \mid \theta_{j}=\theta_{j}^{\star}, 2 \leq j \leq d, y\right)=p\left(y \mid \theta=\left(\theta_{1}, \theta_{2}^{\star}, \ldots \theta_{d}^{\star}\right)^{\prime}\right) \cdot \pi\left(\theta=\left(\theta_{1}, \theta_{2}^{\star}, \ldots \theta_{d}^{\star}\right)^{\prime}\right)
$$

In Section 4.5, we will use this method to derive the full conditional posterior distributions for all parameters in the proposed hierarchical model in Equation (4.4). 


\subsection{Approaches to Simulating from the Posterior Distribution}

When there are many parameters or if the prior is very diffuse compared to the likelihood, direct Monte Carlo sampling from the posterior is very inefficient. Because a huge prior sample size is necessary to get a reasonable sized sample from the posterior. In this section, we will look at MCMC methods for generating samples from the posterior distribution.

MCMC methods sample successively from a target distribution. Each sample depends on the previous one, hence the notion of the Markov chain. Here we don't draw our sample from the posterior distribution directly. Rather we set up a Markov chain that has the posterior distribution as its limiting distribution. Surprisingly MCMC methods are more efficient than the direct procedures for drawing samples from the posterior when we have a large number of parameters. We run the chain long enough so that it approaches the long-run distribution. A value taken after that is a draw from the long-run distribution. Thus we want to find a Markov chain that has the posterior distribution of the parameters given the data as its long-run distribution. We need to find a probability transition kernel $P(\theta, A)$ that satisfies

$$
\int f(\theta \mid y) \cdot P(\theta, A) d \theta=\int_{A} f(\theta \mid y) d \theta
$$

for all $A$ (measurable subsets of parameter space) where $P(\theta, A)$ is the transition kernel of the chain. The left side of this equation is the total steady state probability of set $A$ while the right side of the equation is the steady state flow of probability into set $A$. 
The Markov chain method is very successful in modern Bayesian computing. Since in the simplest Bayesian models can you recognize the analytical forms of the posterior distributions and summarize inferences directly. In moderately complex models, posterior densities are too difficult to work with directly. With the MCMC method, it is possible to generate samples from an arbitrary posterior density and to use these samples to approximate expectations of quantities of interest. More importantly Markov chain is able to improve its approximation to the true distribution at each step in the simulation. Furthermore, if the chain is run for a very long time (often required), you can recover $P(\theta \mid y)$ to any precision.

\subsubsection{Metropolis-Hastings Algorithm}

The Metropolis-Hastings (M-H) algorithm is named after its inventor, the American physicist and computer scientist Nicholas C. Metropolis. The algorithm is simple but practical, and it can be used to obtain random samples from any arbitrarily complicated target distribution of any dimension that is known up to a normalizing constant. The algorithm can be used to generate sequences of samples from the joint distribution of multiple variables, and it is the foundation of MCMC.

The main idea is to generate Markov Chain $\left\{\theta_{t} \mid t=0,1,2, \ldots\right\}$ such that its stationary distribution is the target distribution. The algorithm must specify, for given state $\theta_{t}$, how to generate the next state $\theta_{t+1}$. In all of the $\mathrm{M}-\mathrm{H}$ sampling algorithms, there is a candidate point Y generate from a proposal distribution $f\left(\cdot \mid \theta_{t}\right)$. If this candidate point is accepted, the chain moves to state $Y$ at time $t+1$ and $\theta_{t+1}=Y$; otherwise the chain stay in the 
state $\theta_{t}$ and $\theta_{t+1}=\theta_{t}$. Note that the proposal distribution can depend on the previous state $\theta_{t}$.

The choice of proposal distribution is very flexible, but the chain generated by this choice must satisfy certain regularity conditions. The proposal distribution must be chosen so that the generated chain will converge to a stationary distribution, the target distribution $f$. A proposal distribution with the same support set as the target distribution will usually satisfy these regularity conditions.

The Metropolis-Hastings Sampler Algorithm to generate the Markov chain $\left\{\theta_{0}, \theta_{1}, \ldots\right\}$ is as follows :

1. Choose a proposal distribution $g\left(\cdot \mid \theta_{t}\right)$.

2. Generate $\theta_{0}$ from a distribution $g$.

3. Repeat (until the chain has converged to a stationary distribution according to some criterion):

(a) Generate $Y$ from $g\left(\cdot \mid \theta_{t}\right)$.

(b) Generate $U$ from Uniform $(0,1)$.

(c) If

$$
U \leq \frac{f(Y) g\left(\theta_{t} \mid Y\right)}{f\left(\theta_{t}\right) g\left(Y \mid \theta_{t}\right)}
$$

accept $Y$ and set $\theta_{t+1}=Y$; otherwise set $\theta_{t+1}=\theta_{t}$.

(d) Increment $t$.

Observe that in step (3c) the candidate point $Y$ is accepted with probability

$$
\alpha\left(\theta_{t}, Y\right)=\min \left(1, \frac{f(Y) g\left(\theta_{t} \mid Y\right)}{f\left(\theta_{t}\right) g\left(Y \mid \theta_{t}\right)}\right)
$$


so that it is only necessary to know the density of the target distribution $f$ up to a constant.

Assuming that the proposal distribution satisfies the regularity conditions, the MetropolisHastings chain will converge to a unique stationary distribution $\pi$. The algorithm is designed so that the stationary distribution of the Metropolis-Hastings chain is indeed the target distributions, $f$.

\subsubsection{Gibbs Sampler}

The Gibbs sampler, named by Geman \& Geman (1984) after the American physicist Josiah W. Gibbs, is a special case of the Metropolis sampler in which the proposal distributions exactly match the posterior conditional distributions, and the proposal distributions would be accepted $100 \%$ of the time. Gibbs sampler requires to decompose the joint posterior distribution into full conditional distributions for each parameter in the model and then sample from them (using method in Section 2.3). The sampler can be efficient when the parameters are not highly dependent on each other and the full conditional distributions are easy to sample from. Some researchers favor this algorithm because it does not require an instrumental proposal distribution as Metropolis methods do. And it is often applied when the target is a multivariate distribution. Suppose that all the univariate conditional densities are fully specified and it is reasonably easy to sample from them. The chain is generated by sampling from the marginal distributions of the target distribution, and every candidate point is therefore accepted. However, while deriving the conditional distributions can be relatively easy, it is not always possible to find an 
efficient way to sample from these conditional distributions.

Let $\theta=\left(\theta_{1}, \ldots, \theta_{d}\right)$ be a random vector in $\mathbb{R}^{d}$. Define the $d-1$ dimensional random vectors

$$
\theta_{(-j)}=\left(\theta_{1}, \theta_{2}, \ldots, \theta_{j-1}, \theta_{j+1}, \ldots, \theta_{d-1}, \theta_{d}\right)
$$

and denote the corresponding uni variate conditional density of $\theta_{j}$ given $\theta_{(-j)}$ by $f\left(\theta_{j} \mid\right.$ $\left.\theta_{(-j)}\right)$. The Gibbs sampler generates the chain by sampling from each of the $d$ conditional densities $f\left(\theta_{j} \mid \theta_{(-j)}\right)$.

The Gibbs Sampler Algorithm is as follows, we denote $\theta_{t}$ by $\theta(t)$ for time $t$ :

1. Initialize $\theta(0)$ at time $t=0$.

2. For each iteration, indexed $t=1,2, \ldots$ repeat:

(a) Set $x_{1}=\theta_{1}(t-1)$.

(b) For each coordinate $j=1, \ldots, d$

i. Generate $\theta_{j}^{\star}(t)$ from $f\left(\theta_{j} \mid x_{(-j)}\right)$.

ii. Update $x_{j}=\theta_{j}^{\star}(t)$.

(c) Set $\theta(t)=\left(\theta_{1}^{\star}(t), \ldots, \theta_{d}^{\star}(t)\right)$ (every candidate is accepted).

(d) Increment $t$.

\subsubsection{Other Methods}

\section{Random Walk Metropolis}

The random walk Metropolis sampler is an example of a Metropolis sampler. Suppose the candidate point $Y$ is generated from a symmetric proposal distribution $g\left(Y \mid \theta_{t}\right)=$ $g\left(\left|X_{t}-Y\right|\right)$. Then at each iteration, a random increment $Z$ is generated from $g(\cdot)$, and $Y$ is defined by $Y=\theta_{t}+Z$. For example, the random increment might be normal with mean zero, so that the candidate point $Y \mid \theta_{t} \sim \mathrm{N}\left(\theta_{t}, \sigma^{2}\right)$ for some fixed $\sigma^{2}>0$. 
Convergence of the random walk Metropolis is often sensitive to be choice of scale parameter. When variance of the increment is too large, most of the candidate points are rejected and the algorithm is very inefficient. If the variance of the increment is too small, the candidate points are almost all accepted, so the random walk Metropolis generates a chain that is almost like a true random walk, which is also inefficient.

\section{The Independence Sampler}

Another special case of Metropolis-Hastings sampler is the independence sampler. It is called the independence sampler because the proposal distribution in the algorithm does not depend on the current point as it does with the random-walk Metropolis algorithm. For this sampler to work well, we need to have a proposal density distribution is a close match to the target distribution and have the acceptance rate be as high as possible.

However, a good proposal density should have thicker tails than those of the target distribution. This requirement sometimes can be difficult to satisfy especially in cases where you do not know what the target posterior distributions are like. In addition, independence sampler does not produce independent samples as the name seems to imply, and sample chains from independence samplers can get stuck in the tails of the posterior distribution if the proposal distribution is not chosen carefully. 


\section{CHAPTER 3}

\section{REVIEW OF RELEVANT LITERATURE FOR MULTIPLE COMPARISONS}

\subsection{Introduction}

During the history of statistics, two major schools of thought emerged along the way and have been locked in an on-going struggle in trying to determine which one has the correct view on probability. These two schools are known as the frequentist and Bayesian schools of thought. Both the frequentist and Bayesians hold a different philosophical view on what defines probability and even statistical analysis methodology. They address the same problem with different calculations, and can reach substantially different conclusions even in the absence of strong prior information. By a mathematical coincidence, the results of frequentist and Bayesian calculations for some of the most simple and most common problems are mathematically identical. However, they differ, not only in their interpretations, but in their derivations, and such an identity will not hold in general.

The key contrast between frequentist and Bayesian methods is not the use of prior 
information, but rather the choice of alternatives that are relevant for inference: Bayesian inference focuses on alternative hypotheses, frequentist statistics focuses on alternative data. To assess an hypothesis, $H_{1}$, frequentist methods assume $H_{1}$ is true, and compare the probability of the observed data, $D$, with the probabilities of other data sets predicted by $H_{1}$; Bayesian methods compare the probability of $H_{1}$ with the probabilities of other hypotheses with priors for the hypotheses which determine precisely how much $H_{1}$ is true, Loredo, T. J. (1992).

This chapter provides an overall review to different types of multiple comparison methods in both frequentist and Bayesian methods. We start with discussing simultaneous statistical inference of one-way model in Section 3.2. In Section 3.3, we describe two commonly used multiple comparison inferences, multiple comparisons with a control (MCC) and all-pairwise comparisons (MCA) in the frequentist method. Several well-established methods: Tukey's method (1953) and Dunnet's method (1955) are described as well. In Section 3.4, the notion of a Bayesian factor in the setting of one testing hypothesis is proposed, then the computation of the Bayes factors in the one -sided setting is introduced. At last, in Section 3.5, an example shows the differences between the frequentist and the Bayesian methods. 


\subsection{Frequentist Methods}

Frequentist methods such as confidence intervals and p-values have the advantage that they perform well without knowledge of such a distribution of the parameters. The probability $\mathrm{P}(\mathrm{A})$ is the long-run relative frequency with which A occurs in an infinite number of repeated experiments, or throughout an infinite ensemble. With this understanding of probability, the argument of a probability symbol cannot be an arbitrary proposition, but must be a proposition about a random variable, a quantity that can meaningfully be considered to vary throughout a series of repeated experiments or throughout a physically meaningful ensemble. For example, a coin with a $50 \%$ probability of heads will turn up heads $50 \%$ of the time. Also, any statistical process only has interpretations based on limited frequencies. For example, a 95\% confidence interval of a given parameter will contain the true value of the parameter $95 \%$ of the time; a particular hypothesis is typically either true or false in every repetition of an experiment. Its frequentist probability, to the extent that it is meaningful, is either one or zero.

In the following two sections, two popular frequentist multiple comparison methods are discussing in details: Multiple Comparisons with a Control (MCC) and All-pairwise Comparisons (MCA). 


\subsubsection{Simultaneous Statistical Inference}

Give a one-way model, denote by:

$$
Y_{i j}=\mu_{i}+\epsilon_{i j}, i=1, \ldots, n_{i}
$$

where $Y_{i j}$ is the $j_{t h}$ response for $i_{t h}$ treatment, and $\epsilon_{i j}^{\prime}$ are independent and identically distributed normal errors with mean 0 and variance $\sigma^{2}$. Sample mean measurement is denoted by $\bar{Y}_{i}$, which is a least square estimate for $\mu_{i}$. The pooled sample variance is an unbiased estimator of $\sigma^{2}$. They are given by

$$
\begin{gathered}
\hat{\mu}_{i}=\bar{Y}_{i}=\frac{1}{n} \sum_{j=1}^{n} Y_{i j}, \\
\hat{\sigma}^{2}=\sum_{i=1}^{k} \sum_{j=1}^{n}\left(Y_{i j}-\hat{Y}_{i}\right)^{2} / k(n-1) .
\end{gathered}
$$

Let $v=k(n-1)$, which is the degrees of freedom associated with $\hat{\sigma}^{2}$.

Multiple comparison inference is simultaneous inference on a comparison of the treatment means. Base on the primary interest of the parameters, multiple comparison methods can be mainly classified into four different types: multiple comparisons with a control (MCC) and all-pairwise comparisons (MCA), multiple comparisons with the best (MCB), and all-contrast comparisons (ACC). Since this dissertation is focus on simple order assumption and none order assumption, we will briefly describe MCC and MCA in the following subsections. Usually the one-way model is assumed for the multiple comparison inference. 


\subsubsection{Multiple Comparisons with a Control (MCC)}

Dunnet (1955) is the pioneer of the concept of MCC. While all pairwise comparisons are not the goal of the multiple comparison procedure, but rather only to compare one of the groups (the "controls" usually) with each other group, MCC reduces the multiplecomparisons price otherwise would pay, while taking into account that all these comparisons are correlated since they all use the same "control" data. The parameter of interests are $\mu_{i}-\mu_{k}$, where $i=1, \ldots, k-1$. If the interest is to test whether any new treatment is better or worse than the control, one-sided MCC is a preferred. If the interest is to detect whether the effect of the new treatment and that of the control are different, two-sided MCC is preferred.

Dunnet's (1955) method gives the following simultaneous confidence interval for $\mu_{i}-\mu_{k}$, where $i=1, \ldots, k-1$,

$$
\mu_{i}-\mu_{k}>\hat{\mu}_{i}-\hat{\mu}_{k} \pm \mathrm{d} \hat{\sigma} \sqrt{2 / n} \quad \text { for } i=1, \ldots, k-1
$$

where $d$ is the solution to the equation

$$
\int_{0}^{\infty} \int_{-\infty}^{\infty}[\Phi(z+\sqrt{2} d s)]^{k-1} \mathrm{~d} \Phi(z) \gamma(s) \mathrm{d} s=1-\alpha .
$$

Here $\Phi(z)$ is the cdf of a standard normal distribution, and $\gamma$ is the density function of $\hat{\sigma} / \sigma$, which is a $\sqrt{\chi_{v}^{2} / v}$ random variable. 
Theorem 3.2.1. (Dunnet, 1955) With d defined in the one-sided MCC above,

$$
\begin{aligned}
& P\left\{\mu-\mu_{k}>\hat{\mu}_{i}-\hat{\mu}_{k}-d \hat{\sigma} \sqrt{2 / n}, \quad \text { for } i=1, \ldots, k-1\right\} \\
& =P\left\{\mu-\mu_{k}>\hat{\mu}_{i}-\hat{\mu}_{k}+d \hat{\sigma} \sqrt{2 / n}, \quad \text { for } i=1, \ldots, k-1\right\} \\
& =1-\alpha .
\end{aligned}
$$

Proof. $P\left\{\mu-\mu_{k}>\hat{\mu}_{i}-\hat{\mu}_{k}-d \hat{\sigma} \sqrt{2 / n}, \quad\right.$ for $\left.i=1, \ldots, k-1\right\}$

$$
\begin{aligned}
& =E\left[E\left[P\left\{\frac{\sqrt{n}\left(\hat{\mu}_{i}-\mu_{i}-\left(\hat{\mu}_{k}-\mu_{k}\right)\right) / \sigma}{\hat{\sigma} / \sigma}<d \sqrt{2}, \text { for } i=1, \ldots, k-1\right\} \mid \hat{\mu}_{k}\right] \mid \hat{\sigma}\right] \\
& =\int_{0}^{\infty} \int_{-\infty}^{\infty}[\Phi(z+\sqrt{2} d s)]^{k-1} \mathrm{~d} \Phi(z) \gamma(s) \mathrm{d} s \\
& =1-\alpha
\end{aligned}
$$

Similarly, $P\left\{\mu-\mu_{k}>\hat{\mu}_{i}-\hat{\mu}_{k}+d \hat{\sigma} \sqrt{2 / n}, \quad\right.$ for $\left.i=1, \ldots, k-1\right\}=1-\alpha$.

Dunnet's (1955) method for two-sided case gives the following simultaneous confidence for the difference between each treatment mean $\mu_{i}$ and the control mean $\mu_{k}$ under the balanced one-way model, it is given by

$$
\mu_{i}-\mu_{k} \in \hat{\mu}_{i}-\hat{\mu}_{k} \pm|\mathrm{d}| \hat{\sigma} \sqrt{2 / n} \quad \text { for } i=1, \ldots, k-1
$$

where $|d|$ is the solution to the equation

$$
\int_{0}^{\infty} \int_{-\infty}^{\infty}[\Phi(z+\sqrt{2}|d| s)-\Phi(z-\sqrt{2}|d| s)]^{k-1} \mathrm{~d} \Phi(z) \gamma(s) \mathrm{d} s=1-\alpha
$$


Theorem 3.2.2. (Dunnet, 1955) With d defined in the two-sided MCC above,

$$
\begin{aligned}
& \qquad P\left\{\mu-\mu_{k}>\hat{\mu}_{i}-\hat{\mu}_{k} \pm|d| \hat{\sigma} \sqrt{2 / n}, \quad \text { for } \quad i=1, \ldots, k-1\right\}=1-\alpha . \\
& \text { Proof. } P\left\{\mu-\mu_{k}>\hat{\mu}_{i}-\hat{\mu}_{k} \pm|d| \hat{\sigma} \sqrt{2 / n}, \quad \text { for } i=1, \ldots, k-1\right\} \\
& =E\left[E\left[P\left\{-d \sqrt{2}<\frac{\sqrt{n}\left(\hat{\mu}_{i}-\mu_{i}-\left(\hat{\mu}_{k}-\mu_{k}\right)\right) / \sigma}{\hat{\sigma} / \sigma}<d \sqrt{2} \quad \text { for } i=1, \ldots, k-1\right\} \mid \hat{\mu}_{k}\right] \mid \hat{\sigma}\right] \\
& =\int_{0}^{\infty} \int_{-\infty}^{\infty}[\Phi(z+\sqrt{2} d s)-\Phi(z-\sqrt{2} d s)]^{k-1} \mathrm{~d} \Phi(z) \gamma(s) \mathrm{d} s \\
& =1-\alpha .
\end{aligned}
$$

In essence, the one-sided Dunnett's test takes the minimum (or the maximum, depending on the sideness of the test problem) of the $m$, say, pairwise $t$ tests

$$
t_{i}=\frac{\bar{Y}_{i}-\bar{Y}_{0}}{s \sqrt{\frac{1}{\frac{1}{n_{i}}+\frac{1}{n_{0}}}}}, \quad i=1, \ldots, m
$$

where $\bar{Y}_{i}=\frac{1}{n_{i}} \sum_{j=1}^{n_{i}} Y_{i j}$ denotes the arithmetic mean of group $i=0, \ldots, m$ and $s=$ $\sum_{i=1}^{k} \sum_{j=1}^{n_{i}}\left(Y_{i j}-\hat{Y}_{i}\right)^{2} / k(n-1)$ denotes the pooled variance estimate with $v=k(n-1)$, which is the degrees of freedom associated with $\hat{\sigma}^{2}$.

The advantage of Dunnet's method is if the procedure is really interested only in comparisons of one group to each of the others, this approach is more powerful than methods performing all possible pairwise comparisons, therefore is recommended. Its disadvantage is simply that it does not compare the "other" groups to each other at all, it may cause some results are undetected. 


\subsubsection{All-pairwise Comparisons (MCA)}

For MCA, the parameter of interest is $\mu_{i}-\mu_{j}$, for all $i \neq j$. There are totally $k(k-$ 1)/2 pairwise differences of treatment means. Tukey's (1953) approach is specifically for comparing group means in an ANOVA setting. It is based on the distribution of q, the "studentized range." The "studentized range" with $\mathrm{k}$ and $\mathrm{r}$ degrees of freedom is the range (i.e. maximum - minimum) of a set of $\mathrm{k}$ independent observations from some normal distribution, divided by an independent estimate (with $\mathrm{r}$ degrees-of-freedom) of the standard deviation of that normal distribution. This approach is considered as the best for all-possible pairwise comparisons when sample sizes are unequal or confidence intervals are needed; It is very good even with equal samples sizes without confidence intervals.

Tukey's simultaneous confidence intervals for all-pairwise differences:

$$
\left.\mu_{i}-\mu_{j}>\hat{\mu}_{i}-\hat{\mu}_{j} \pm\left|q^{\star}\right| \hat{\sigma} \sqrt{2 / n} \quad \text { for all } i, j, \text { where } i \neq j\right\}
$$

where $\left|q^{\star}\right|$ is the solution to the equation

$$
=P\left\{\frac{\left|\left(\hat{\mu}_{i}-\mu_{i}\right)-\left(\hat{\mu}_{j}-\mu_{j}\right)\right|}{\hat{\sigma} \sqrt{2 / n}}<\left|q^{\star}\right| \text { for all } i \neq j\right\}=1-\alpha .
$$

Numerically, $\left|q^{\star}\right|$ is the solution to the equation

$$
k \int_{0}^{\infty} \int_{-\infty}^{\infty}\left[\Phi(z)-\Phi\left(z-\sqrt{2}\left|q^{\star}\right| s\right)\right]^{k-1} \mathrm{~d} \Phi(z) \gamma(s) \mathrm{d} s=1-\alpha
$$


where $\Phi(z)$ is the cdf of a standard normal distribution, and $\gamma$ is the density function of $\hat{\sigma} / \sigma$, which is a $\sqrt{\chi_{v}^{2} / v}$ random variable. $s$ is the pooled sample standard deviation. Therefore, with d defined in the two-sided MCC above, we have

$$
P\left\{\mu_{i}-\mu_{j} \in \hat{\mu}_{i}-\hat{\mu}_{j} \pm\left|q^{\star}\right| \hat{\sigma} \sqrt{2 / n}, \quad \text { for all } \mathrm{i} \neq \mathrm{j}\right\}=1-\alpha \text {. }
$$

Theorem 3.2.3. For all $\mu, \sigma^{2}$,

$$
P\left\{\mu_{i}-\mu_{j} \in \hat{\mu}_{i}-\hat{\mu}_{j} \pm\left|q^{\star}\right| \hat{\sigma} \sqrt{2 / n}, \quad \text { for all } i \neq j\right\}=1-\alpha
$$

where $\left|q^{\star}\right|$ is the solution to the equation

$$
k \int_{0}^{\infty} \int_{-\infty}^{\infty}\left[\Phi(z)-\Phi\left(z-\sqrt{2}\left|q^{\star}\right| s\right)-\Phi(z-\sqrt{2}|d| s)\right]^{k-1} d \Phi(z) \gamma(s) d s=1-\alpha
$$

Proof. $P\left\{\mu_{i}-\mu_{j} \in \hat{\mu}_{i}-\hat{\mu}_{j} \pm\left|q^{\star}\right| \hat{\sigma} \sqrt{2 / n}, \quad\right.$ for all $\left.i \neq j\right\}$

$$
\begin{aligned}
& =\sum_{i=1}^{k} P\left\{-\left|q^{\star}\right| \hat{\sigma} \sqrt{2 / n}<\left(\left(\hat{\mu}_{i}-\hat{\mu}_{j}\right)-\left(\mu_{i}-\mu_{j}\right)\right)<\left|q^{\star}\right| \hat{\sigma} \sqrt{2 / n},\right. \\
& \left.\quad \text { for all } i \neq j, \text { and } \hat{\mu}_{i}-\mu_{i}=\max _{j=1, \ldots, k}\left(\hat{\mu}_{j}-\mu_{j}\right)\right\} \\
& =\sum_{i=1}^{k} P\left\{0<\left(\left(\hat{\mu}_{i}-\hat{\mu}_{j}\right)-\left(\mu_{i}-\mu_{j}\right)\right)<\left|q^{\star}\right| \hat{\sigma} \sqrt{2 / n}, \quad \text { for all } i \neq j\right\} \\
& =\sum_{i=1}^{k} P\left\{0<\left(\left(\hat{\mu}_{i}-\mu_{i}\right)-\left(\hat{\mu}_{j}-\mu_{j}\right)\right)<\left|q^{\star}\right| \hat{\sigma} \sqrt{2 / n}, \quad \text { for all } i \neq j\right\} \\
& =\sum_{i=1}^{k} P\left\{0<\left(\frac{\sqrt{n}}{\sigma}\left(\hat{\mu}_{i}-\mu_{i}\right)-\frac{\sqrt{n}}{\sigma}\left(\hat{\mu}_{j}-\mu_{j}\right)\right)<\left|q^{\star}\right| \frac{\hat{\sigma} \sqrt{2 / n}}{\sigma}, \quad \text { for all } i \neq j\right\}
\end{aligned}
$$




$$
\begin{aligned}
& =k \cdot P\left\{0<\left(\frac{\sqrt{n}}{\sigma}\left(\hat{\mu}_{i}-\mu_{i}\right)-\frac{\sqrt{n}}{\sigma}\left(\hat{\mu}_{j}-\mu_{j}\right)\right)<\left|q^{\star}\right| \frac{\hat{\sigma} \sqrt{2 / n}}{\sigma}, \quad \text { for } j=2, \ldots, k\right\} \\
& =k \int_{0}^{\infty} \int_{-\infty}^{\infty}\left[\Phi(z)-\Phi\left(z-\sqrt{2}\left|q^{\star}\right| s\right)-\Phi(z-\sqrt{2}|d| s)\right]^{k-1} \mathrm{~d} \Phi(z) \gamma(s) \mathrm{d} s \\
& =1-\alpha .
\end{aligned}
$$

In essence, the Tukey test takes the maximum over the absolute values of all pairwise test statistics, which are in the completely randomized layout that take the form

$$
t_{i j}=\frac{\bar{Y}_{i}-\bar{Y}_{j}}{s \sqrt{\frac{2}{n}}}
$$

where $\bar{Y}_{i}$ denotes the least squares mean estimate for variety $i$ (which coincides with the usual arithmetic mean estimate in this case), $s$ is the pooled standard deviation and $n$ is the common sample size.

The advantage of Tukey's method is when doing all pairwise comparisons, it is considered the best available when confidence intervals are needed or sample sizes are not equal. When samples sizes are equal and confidence intervals are not needed Tukey's test is slightly less powerful than the stepdown procedures, but if they are not available, Tukey's is the next-best choice, and unless the number of groups is large, the loss in power will be slight. 


\subsection{Error Rate}

For any test problem, there are three types of errors. A false positive decision occurs if we declare an effect when none exists. Similarly, a false negative decision occurs if we fail to declare a truly existing effect. In hypotheses test problems, these errors are denoted as Type I and Type II errors, respectively. The correct rejection of a null hypothesis coupled with a wrong directional decision is denoted as Type III error. The related notation is summarized in Table 3.1. Let $M=\{1, \ldots, m\}$ denote the index set associated with the null hypotheses $H_{1}, \ldots, H_{m}$ and let $M_{0} \subseteq M$ denote the set of $m_{0}=\left|M_{0}\right|$ true by potheses, where $|A|$ denotes the cardinality of a set $A$. In Table 2.1, $V$ denotes the number of Type I errors and $R$ the number of rejected hypotheses, where $T$ denotes the number of Type II errors, and $R$ the number of rejected hypotheses. Note that $R$ is an observable random variable, $S, T, U$, and $V$ are all unobservable random variables, while $m$ and $m_{0}$ are fixed numbers, where $m_{0}$ is unknown.

Table 3.1: Type I and II Errors in Multiple Hypotheses Testing

\begin{tabular}{cccc}
\hline Hypotheses & Not Rejected & Rejected & Total \\
\hline True & $U$ & $V$ & $m_{0}$ \\
False & $T$ & $S$ & $m-m_{0}$ \\
\hline Total & $W$ & $R$ & $m$ \\
\hline
\end{tabular}




\subsection{Family-wise Error Rate (FWER)}

Usually, the purpose of multiple comparisons is to compare several treatment effects by estimating the difference or by testing a family of hypothesis. If to test a family of hypothesis or there is no prior expectation of any term in the test group, the control of the family-wise error rate (FWER) is regarded as the appropriate multiple testing correction. The FWER is the probability of rejecting at least one true $H_{i}$ (Hochberg \& Tamhane (1987)). For a given significance level $\alpha$, weak control of FWER means the control of the chance of falsely rejecting any hypothesis when all null hypotheses are actually true; Strong control of FWER means the control of the chance of falsely rejecting any hypothesis regardless of how many hypotheses are true, which is denoted by:

$$
P\left(\text { reject at least one } H_{i}, i \in I \mid H_{i}, i \in I \text { is true }\right) \leqslant \alpha \text {, }
$$

where $I$ is a non-empty subset of the index set $\{1,2, \ldots, k\}$. In this research, FWER is commonly used in multiple comparisons in simulations and applications.

The false discovery rate (FDR) is a different way to explain how the Type I error could be considered in multiple testing, and it is designed to control the expected proportion of errors among the rejected hypotheses (Benjamini \& Hochberg (1995)). For a given significance level $\alpha$, if all null hypotheses are true, then the FDR is equivalent to the FWER; Otherwise, the FDR is less or equal to the FWER. Thus, controlling the FWER in any procedure would also control the FDR. Therefore, we will only focus on the FWER in this dissertation. 


\subsection{Bayesian Methods}

To introduce Bayesian method for model comparison, suppose one observes Y from sampling distribution $f(y \mid \theta)$ and to test the hypothesis

$$
H_{0}: \theta \in \Theta_{0}, \quad H_{1}: \theta \in \Theta_{1},
$$

where $\Theta_{0}$ and $\Theta_{1}$ form a partition of the parameter space. If a proper prior density $g(\theta)$ has been assigned, then we can judge the two hypotheses a priori by the prior odds ratio

$$
\frac{\pi_{0}}{\pi_{1}}=\frac{P\left(\theta \in \Theta_{0}\right)}{P\left(\theta \in \Theta_{1}\right)}=\frac{\int_{\Theta_{0}} g(\theta) d \theta}{\int_{\Theta_{1}} g(\theta) d \theta} .
$$

After data $Y=y$ is observed, our beliefs about the parameter now update by the posterior density

$$
g(\theta \mid y) \propto L(\theta) g(\theta)
$$

where $L(\theta)$ is the likelihood function. Now our new beliefs about the two hypotheses are summarized by the posterior odds ratio

$$
\frac{\pi_{0}}{\pi_{1}}=\frac{P\left(\theta \in \Theta_{0}\right)}{P\left(\theta \in \Theta_{1}\right)}=\frac{\int_{\Theta_{0}} g(\theta) d \theta}{\int_{\Theta_{1}} g(\theta) d \theta} .
$$

The Bayes factor is the ratio of the posterior odds to the prior odds of the hypotheses

$$
B F=\frac{\text { posterior odds }}{\text { prior odds }}=\frac{p_{0} / p_{1}}{\pi_{0} / \pi_{1}}
$$


The statistic BF is a measure of the evidence provided by the data in support of the hypothesis $H_{0}$. The posterior probability of the hypothesis $H_{0}$ can be expressed as a function of the Bayes factor and the prior probabilities of the hypotheses by

$$
p_{0}=\frac{\pi_{0} B F}{\pi_{0} B F+1-\pi_{0}} .
$$

\subsection{Example}

Berry (1996) was interested in determining his true weight from a variable bathroom scale. Assume the measurements are normally distributed with mean $\mu$ and standard deviation $\sigma$. Then the author weighs himself 10 times with the measurements (in pounds) 182, $172,173,176,176,180,173,174,179$, and 175 . For simplicity, assume that he knows the accuracy of the scale and $\sigma=3$ pounds.

Let $\mu$ denote the author's true weight, suppose he is interested in assessing if his true weight is greater than 175 pounds. Then the test hypotheses

$$
H_{0}: \mu \leq 175, H_{1}: \mu>175
$$

Suppose the author has knowledge about his true weight and $\mu \sim \mathrm{N}(170,5)$, which is mean 170 pounds and standard deviation 5 pounds.

The prior odds of the null hypothesis $H_{0}$ is given by

$$
\frac{\pi_{0}}{\pi_{1}}=\frac{P(\mu \leq 175)}{P(\mu>175)} .
$$


Next we compute the prior odds from the $\mathrm{N}(170,5)$ density using normal distribution, prior odds $=5.3029$, so a priori the null hypothesis is five times more likely than the alternative hypothesis.

Then we use the 10 weight measurements to compute the sample mean and associated sampling variance. By Bayesian method, we can calculate the posterior density of $\mu$ is $\mathrm{N}(175.79,0.93)$. Then using this normal posterior density, calculate the posterior odds of the null hypothesis, posterior odds $=0.2467$, then

$$
B F=\frac{\text { posterior odds }}{\text { prior odds }}=\frac{0.2467}{5.3029}=0.04652 \text {. }
$$

From the prior probabilities and the Bayes factor, we can compute the posterior probability of the null hypothesis,

$$
p_{0}=\frac{\pi_{0} B F}{\pi_{0} B F+1-\pi_{0}}=\frac{0.84134 * 0.04652}{0.84134 * 0.04652+1-0.84134}=0.1978
$$

base on this result, we can conclude that it is unlikely that the author's weight is at most 175 pounds, we are in favor of the alternative hypothesis that his weight is more than 175 pounds.

For the frequentist method, here with a known value of the standard deviation $\sigma$, the traditional test of $H_{0}$ is based on the test statistic

$$
z=\frac{(\bar{y}-175)}{3 / \sqrt{n}}
$$


where $\bar{y}$ is the sample mean and $n$ is the sample size. Then we calculate the $z$-score and find the $p$-value $=0.1459$.

There is an interesting connection between this Bayesian measure of evidence and the frequentist $p$-value. Even though there is significant difference between the two results above, however, if we repeat the Bayesian analysis using a very flat prior where the mean and standard deviation are 170 and 1000, respectively.

Table 3.2: $p$-value Comparison in Bayesian and Frequentist Method for Height Example

\begin{tabular}{ccc}
\hline & Bayesian Method & Frequentist Method \\
\hline \hline Bayes factor $(\mathrm{BF})$ & 0.1695 & \\
\hline posterior odds & 0.1709 & \\
\hline prior odds & 1.0080 & 0.1459 \\
\hline probability $/ p$-value & 0.1459 & \\
\hline
\end{tabular}

We can see the probability of the null hypothesis is approximately equal to the $p$-value. This illustrates a general result that a Bayesian probability of a hypothesis is equal to the $p$-value for one-sided testing problems when a vague prior distribution is placed on the parameter. (The R-code for this example is in the first section of Appendix.) 


\section{CHAPTER 4}

\section{DERIVATION OF THE BAYESIAN}

\section{HIERARCHICAL MODEL FOR}

\section{MULTIPLE COMPARISONS IN MIXED}

\section{MODELS}

\subsection{Introduction}

A Bayesian hierarchical model can view as a problem with three entities, all of which have stochastic elements. First is the data which is presumed to be drawn from a sample. Second is the process specification itself which involves unknowns that will be estimated as parameters. Third, we have parameters that are not only "uncertain" but will be expected to vary depending upon how and where the data were obtained. With this three-parts structure in mind, we are prepared to extend the earlier idea of the Bayesian model to more levels in a general and flexible way, Clard J. S. and Gelfand A. E. (2006).

In this chapter, we focus on deriving the Bayesian hierarchical model in mixed models 
under a two-sided pairwise assumption. In Section 4.2, we present the mixed model which is a two-way ANOVA model. In Section 4.3, we obtain the joint density function of the data vector $Y$. Then we propose and present the utilized priors and hyper-priors density function using the joint density function in Section 4.4. In Section 4.5 we obtain the full conditional posterior distributions for the parameters. In Section 4.6 we present the algorithm for the Gibbs sampler method in order to perform the estimation of parameters and posterior probabilities for multiple comparisons.

\subsection{The Mixed Model}

The two-way ANOVA mixed model is defined as

$$
y_{i j}=\mu_{i}+b_{j}+\varepsilon_{i j}, \quad i=1, \ldots, k, j=1, \ldots, m
$$

where $y_{i j}, u_{i}, b_{j}$ and $\varepsilon_{i j}$ are all scalars,

$y_{i j}$ is the response observed on the $j$-th subject under the $i$-th treatment;

$\mu_{i}$ is a fixed treatment effect for the $i$-th treatment;

$b_{j}$ is a random subject effect, which $b_{j} \sim \mathrm{N}\left(0, \sigma_{\tau}^{2}\right)$;

$\varepsilon_{i j}$ is an error term, which $\varepsilon_{i j} \sim \mathrm{N}\left(0, \sigma^{2}\right)$; and the $m(k+1)$ variables $b_{j}$ and $\varepsilon_{i j}, i=1, \ldots, k$ and $j=1, \ldots, m$, are all independent. 
We can re-express model above in more succinct format by writing

$$
y_{j}=\mu+z b_{j}+\varepsilon_{j}, \quad j=1, \ldots, m
$$

where $y_{j}, \mu, z$ and $\varepsilon_{j}$ are all vectors, and $b_{j}$ is a scalar,

$y_{j}$ denotes a $k \times 1$ vector of responses observed on the $j$-th subject;

$\mu$ is the $k \times 1$ vector $\left(\mu_{1}, \mu_{2}, \ldots, \mu_{k}\right)^{\prime}$;

$z$ is a $k \times 1$ vector consisting of all 1 's;

$b_{j}$ is a random subject effect, which $b_{j} \sim \mathrm{N}\left(0, \sigma_{\tau}^{2}\right)$; and

$\varepsilon_{j}$ is a $k \times 1$ distributed as $\varepsilon_{i j} \sim \mathrm{N}\left(0, \sigma^{2} I\right)$.

\subsection{Joint Density Function}

First we need to obtain the density function for the data. By (4.2),

$$
y_{j} \mid \mu, \sigma_{\tau}^{2}, \sigma^{2} \sim \mathrm{N}\left(\mu, z \sigma_{\tau}^{2} z^{\prime}+\sigma^{2} I\right), \quad j=1, \ldots, m
$$

Let $Y=\left(y_{1}^{\prime}, \ldots, y_{m}^{\prime}\right)^{\prime}$. Given that the cases are independent, the joint density function of the data vector $Y$ is given by

$$
\left[Y \mid \mu_{1},\left\{\delta_{i}\right\}, \sigma_{\tau}^{2}, \sigma^{2}\right] \propto\left(\sigma^{2}\right)^{-\frac{m(k-1)}{2}}\left(k \sigma_{\tau}^{2}+\sigma^{2}\right)^{-\frac{m}{2}} \exp \left\{-\frac{1}{2 \sigma^{2}}\left[s_{1}-\frac{\sigma_{\tau}^{2}}{k \sigma_{\tau}^{2}+\sigma^{2}} s_{2}\right]\right\}
$$

where $s_{1}=\sum_{j=1}^{m} \sum_{i=1}^{k}\left(y_{i j}-\mu_{1}-\sum_{l=0}^{i-1} \delta_{l}\right)^{2}$ and $s_{2}=\left(\sum_{j=1}^{m} \sum_{i=1}^{k}\left(y_{i j}-\mu_{1}-\sum_{l=0}^{i-1} \delta_{l}\right)\right)^{2}$, and $\delta_{i}=\mu_{i+1}-\mu_{i}(1<i<k-1)$. 
From $y_{j} \mid \mu, \sigma_{\tau}^{2}, \sigma^{2} \sim \mathrm{N}\left(\mu, z \sigma_{\tau}^{2} z^{\prime}+\sigma^{2} I\right)$, we have

$$
\begin{aligned}
& f\left(y_{j}\right)=\frac{1}{\left|z \sigma_{\tau}^{2} z^{\prime}+\sigma^{2} I\right|^{1 / 2}} \exp \left\{-\frac{\left(y_{j}-\mu\right)^{\prime}\left(y_{j}-\mu\right)}{2\left(z \sigma_{\tau}^{2} z^{\prime}+\sigma^{2} I\right)}\right\} \\
& \quad=\frac{1}{|V|^{1 / 2}} \exp \left\{-\frac{\left(y_{j}-\mu\right)^{\prime} V^{-1}\left(y_{j}-\mu\right)}{2}\right\} \text {, where } V=z \sigma_{\tau}^{2} z^{\prime}+\sigma^{2} I .
\end{aligned}
$$

Because $y_{j}$ is a vector, that is $y_{j}=\left(\begin{array}{c}y_{1 j} \\ y_{2 j} \\ \vdots \\ \vdots \\ y_{k j}\end{array}\right)$, then $y_{j}-\mu=\left(\begin{array}{c}y_{1 j}-\mu_{1} \\ y_{2 j}-\mu_{2} \\ \vdots \\ \vdots \\ y_{k j}-\mu_{k}\end{array}\right)$.

Let $\mathrm{V}=\left(\begin{array}{ccccc}\sigma_{\tau}^{2}+\sigma^{2} & \sigma_{\tau}^{2} & \sigma_{\tau}^{2} & \cdots & \sigma_{\tau}^{2} \\ \sigma_{\tau}^{2} & \sigma_{\tau}^{2}+\sigma^{2} & \sigma_{\tau}^{2} & \cdots & \sigma_{\tau}^{2} \\ \sigma_{\tau}^{2} & \sigma_{\tau}^{2} & \ddots & \ddots & \vdots \\ \vdots & \ddots & \ddots & \ddots & \sigma_{\tau}^{2} \\ \sigma_{\tau}^{2} & \cdots & \cdots & \sigma_{\tau}^{2} & \sigma_{\tau}^{2}+\sigma^{2}\end{array}\right)=\left(\begin{array}{cccc}a & b & \cdots & b \\ b & a & \ddots & \vdots \\ \vdots & \ddots & \ddots & b \\ b & \cdots & b & a\end{array}\right)$

where $a=\sigma_{\tau}^{2}+\sigma^{2}$, and $b=\sigma_{\tau}^{2}$.

Let $\left(y_{j}-\mu\right)^{\prime}=\left(a_{1}, \ldots, a_{k}\right)^{\prime}$, and $V^{-1}=\left(\begin{array}{cccc}c & d & \cdots & d \\ d & c & \ddots & \vdots \\ \vdots & \ddots & \ddots & d \\ d & \cdots & d & c\end{array}\right)$, then for the simplification of notation, we first have the following expression,

$$
\left(a_{1}, \ldots, a_{k}\right) \times\left(\begin{array}{cccc}
c & d & \cdots & d \\
d & c & \ddots & \vdots \\
\vdots & \ddots & \ddots & d \\
d & \cdots & d & c
\end{array}\right) \times\left(\begin{array}{c}
a_{1} \\
\vdots \\
a_{k}
\end{array}\right)
$$




$$
\begin{aligned}
& =\left(a_{1} c+a_{2} d+\cdots+a_{k} d, a_{1} d+a_{2} c+\cdots+a_{k} d, \cdots \cdots, a_{1} d+a_{2} d+\cdots+a_{k} c\right)\left(\begin{array}{c}
a_{1} \\
\vdots \\
a_{k}
\end{array}\right) \\
& =\left(a_{1}^{2} c+a_{1} a_{2} d+a_{1} a_{3} d+\cdots+a_{1} a_{k} d\right. \\
& +a_{1} a_{2} d+a_{2}^{2} c+a_{2} a_{3} d+\cdots+a_{2} a_{k} d \\
& +a_{3} a_{1} c+a_{3} a_{2} d+a_{3}^{2} c+\cdots+a_{3} a_{k} d \\
& \vdots \\
& \vdots \\
& \left.+a_{k} a_{1} c+a_{k} a_{2} d+a_{k} a_{3} d+\cdots+a_{k}^{2} c\right) \\
& =c \sum_{i=1}^{k} a_{i}^{2}+a_{1} d\left(a_{2}+\cdots+a_{k}\right)+a_{2} d\left(a_{1}+a_{3}+\cdots+a_{k}\right)+\cdots+a_{k} d\left(a_{1}+\cdots+a_{k-1}\right) \\
& =c \sum_{i=1}^{k} a_{i}^{2}+a_{1} d\left(a_{1}+a_{2}+\cdots+a_{k}\right)-a_{1} a_{1} d \\
& +a_{2} d\left(a_{1}+a_{2}+a_{3}+\cdots+a_{k}\right)-a_{2} a_{2} d \\
& +a_{k} d\left(a_{1}+\cdots+a_{k-1}+a_{k}\right)-a_{k} a_{k} d \\
& =c \sum_{i=1}^{k} a_{i}^{2}+\left(a_{1} d \sum_{i=1}^{k} a_{i}+a_{2} d \sum_{i=1}^{k} a_{i}+a_{3} d \sum_{i=1}^{k} a_{i}+\cdots+a_{k} d \sum_{i=1}^{k} a_{i}\right) \\
& -\left(a_{1} a_{1} d+a_{2} a_{2} d+\cdots \cdots+a_{k} a_{k} d\right) \\
& =c \sum_{i=1}^{k} a_{i}^{2}+d\left(\sum_{i=1}^{k} a_{i}\right)^{2}-d \sum_{i=1}^{k} a_{i}^{2} \\
& =(c-d) \sum_{i=1}^{k} a_{i}^{2}+d\left(\sum_{i=1}^{k} a_{i}\right)^{2} .
\end{aligned}
$$

By $c=\frac{-a-(k-2) b}{b^{2}(k-1)-a^{2}-(k-2) a b}, d=\frac{b}{b^{2}(k-1)-a^{2}-(k-2) a b}$, and $a=\sigma_{\tau}^{2}+\sigma^{2}$, $b=\sigma_{\tau}^{2}$, we can write the expression of the denominator as 


$$
\begin{aligned}
& b^{2}(k-1)-a^{2}-(k-2) a b \\
= & \left(\sigma_{\tau}^{2}\right)^{2}(k-1)-\left(\sigma_{\tau}^{2}+\sigma^{2}\right)^{2}-(k-2) \cdot \sigma_{\tau}^{2}\left(\sigma_{\tau}^{2}+\sigma^{2}\right) \\
= & \sigma_{\tau}^{4} k-\sigma_{\tau}^{4}-\sigma_{\tau}^{4}-2 \sigma_{\tau}^{2} \sigma^{2}-\sigma^{4}-\sigma_{\tau}^{4} k-\sigma_{\tau}^{2} \sigma^{2} k+2 \sigma_{\tau}^{4}+2 \sigma_{\tau}^{2} \sigma^{2} \\
= & -\sigma^{4}-k \sigma_{\tau}^{2} \sigma^{2} \\
= & -\sigma^{2}\left(k \sigma_{\tau}^{2}+\sigma^{2}\right) .
\end{aligned}
$$

Also we can write the expression of the numerator for $c-d$ as

$$
\begin{aligned}
& -a-(k-2) b-b \\
= & -a-k b+b \\
= & -\left(\sigma_{\tau}^{2}+\sigma^{2}\right)-k\left(\sigma_{\tau}^{2}\right)+\sigma_{\tau}^{2} \\
= & -\left(k \sigma_{\tau}^{2}+\sigma^{2}\right) .
\end{aligned}
$$

Thus, we express $(c-d) \sum_{i=1}^{k} a_{i}^{2}+d\left(\sum_{i=1}^{k} a_{i}\right)^{2}$ as

$$
\begin{aligned}
& \frac{-\left(k \sigma_{\tau}^{2}+\sigma^{2}\right)}{-\sigma^{2}\left(k \sigma_{\tau}^{2}+\sigma^{2}\right)} \sum_{i=1}^{k}\left(y_{i}-\mu\right)^{2}+\frac{\sigma_{\tau}^{2}}{-\sigma^{2}\left(k \sigma_{\tau}^{2}+\sigma^{2}\right)}\left(\sum_{i=1}^{k}\left(y_{i}-\mu\right)\right)^{2} \\
& =\frac{1}{\sigma^{2}}\left[\sum_{i=1}^{k}\left(y_{i}-\mu\right)^{2}-\frac{\sigma_{\tau}^{2}}{k \sigma_{\tau}^{2}+\sigma^{2}}\left(\sum_{i=1}^{k}\left(y_{i}-\mu\right)\right)^{2}\right]
\end{aligned}
$$

Meanwhile, we know that $|V|=(a-b)^{k-1}(a+(k-1) b)$

$$
\begin{aligned}
& =\left(\sigma_{\tau}^{2}+\sigma^{2}-\sigma_{\tau}^{2}\right)^{k-1}\left(\sigma_{\tau}^{2}+\sigma^{2}+k \sigma_{\tau}^{2}-\sigma_{\tau}^{2}\right) \\
& =\left(\sigma^{2}\right)^{k-1}\left(k \sigma_{\tau}^{2}+\sigma^{2}\right) .
\end{aligned}
$$


Thus, we have $f\left(y_{j}\right)=\frac{1}{|V|^{1 / 2}} \exp \left\{-\frac{\left(y_{j}-\mu\right)^{\prime} V^{-1}\left(y_{j}-\mu\right)}{2}\right\}$

$$
\begin{aligned}
& =\frac{1}{\left(\sigma^{2}\right)^{\frac{k-1}{2}}\left(k \sigma_{\tau}^{2}+\sigma^{2}\right)^{1 / 2}} \exp \left\{-\frac{1}{2 \sigma^{2}}\left[\sum_{i=1}^{k}\left(y_{i}-\mu\right)^{2}-\frac{\sigma_{\tau}^{2}}{k \sigma_{\tau}^{2}+\sigma^{2}}\left(\sum_{i=1}^{k}\left(y_{i}-\mu\right)\right)^{2}\right]\right\} \\
& \propto\left(\sigma^{2}\right)^{-\frac{k-1}{2}}\left(k \sigma_{\tau}^{2}+\sigma^{2}\right)^{-\frac{1}{2}} \exp \left\{-\frac{1}{2 \sigma^{2}}\left[\sum_{i=1}^{k}\left(y_{i}-\mu\right)^{2}-\frac{\sigma_{\tau}^{2}}{k \sigma_{\tau}^{2}+\sigma^{2}}\left(\sum_{i=1}^{k}\left(y_{i}-\mu\right)\right)^{2}\right]\right\} .
\end{aligned}
$$

Since $y_{j}$ are independent, the joint density of $Y$ can be expressed as

$$
\begin{aligned}
& {\left[Y \mid \mu, \sigma_{\tau}^{2}, \sigma^{2}\right] \propto \prod_{j=1}^{m} y_{j}} \\
& \propto\left(\sigma^{2}\right)^{-\frac{m(k-1)}{2}}\left(k \sigma_{\tau}^{2}+\sigma^{2}\right)^{-\frac{m}{2}} \exp \left\{-\frac{1}{2 \sigma^{2}}\left[\sum_{j=1}^{m} \sum_{i=1}^{k}\left(y_{i j}-\mu_{i}\right)^{2}-\frac{\sigma_{\tau}^{2}}{k \sigma_{\tau}^{2}+\sigma^{2}}\left(\sum_{j=1}^{m} \sum_{i=1}^{k}\left(y_{i j}-\mu_{i}\right)\right)^{2}\right]\right\} .
\end{aligned}
$$

Let $\mu_{1}$ be the first mean, and define the parameters $\delta_{i}, i=1, \ldots, k-1$ by

$\delta_{i-1}=\mu_{i}-\mu_{i-1}(2<i<k)$.

Thus we have

$$
\begin{aligned}
& \mu_{2}=\mu_{1}+\delta_{1} \\
& \mu_{3}=\mu_{1}+\delta_{1}+\delta_{2} \\
& \vdots \\
& \vdots \\
& \mu_{i}=\mu_{1}+\delta_{1}+\delta_{2}+\cdots+\delta_{i-1}
\end{aligned}
$$

Thus, $\mu_{i}=\mu_{1}+\sum_{l=0}^{i-1} \delta_{l}$.

Let $s_{1}=\sum_{j=1}^{m} \sum_{i=1}^{k}\left(y_{i j}-\mu_{1}-\sum_{l=0}^{i-1} \delta_{l}\right)^{2}$ and $s_{2}=\left(\sum_{j=1}^{m} \sum_{i=1}^{k}\left(y_{i j}-\mu_{1}-\sum_{l=0}^{i-1} \delta_{l}\right)\right)^{2}$,

then 


$$
\left[Y \mid \mu,\left\{\delta_{i}\right\}, \sigma_{\tau}^{2}, \sigma^{2}\right] \propto\left(\sigma^{2}\right)^{-\frac{m(k-1)}{2}}\left(k \sigma_{\tau}^{2}+\sigma^{2}\right)^{-\frac{m}{2}} \exp \left\{-\frac{1}{2 \sigma^{2}}\left[s_{1}-\frac{\sigma_{\tau}^{2}}{k \sigma_{\tau}^{2}+\sigma^{2}} s_{2}\right]\right\}
$$

\subsection{Priors and Hyper-priors}

Our goal is to compare the population means $\mu_{i}, i=1, \ldots, k$ globally and pairwisely using the following proposed Bayesian Hierarchical Model.

To propose a hierarchical model in (4.4), we need to define some priors first. We use a conjugate prior, namely

$$
\mu_{1} \sim \mathrm{N}\left(\mu_{0}, \tau_{0}^{2}\right)
$$

We choose a vague normal prior with an arbitrary mean $\mu_{0}$ and a large variance $\tau_{0}^{2}$.

For the variance components, we consider a non-informative prior distribution as a joint prior for $\sigma_{\tau}^{2}$ and $\sigma^{2}$, and it is expressed as:

$$
\pi\left(\sigma_{\tau}^{2}, \sigma^{2}\right) \propto \frac{1}{\sigma^{2}\left(k \sigma_{\tau}^{2}+\sigma^{2}\right)}
$$

This prior was used by Box \& Tiao (1992) in the balanced mixed model case. The model considered here is also assumed balance, so we can apply this prior to our model. If we let $\tau^{2}=k \sigma_{\tau}^{2}+\sigma^{2}$, then prior distribution is expressed as

$$
\pi\left(\tau^{2}, \sigma^{2}\right) \propto \frac{1}{\sigma^{2} \tau^{2}}
$$

With this prior, we need to obtain the full conditional posterior distribution for $\tau^{2}$ instead of $\sigma_{\tau}^{2}$, and therefore based on $\sigma^{2}$ and $\tau^{2}$, where $\sigma_{\tau}^{2}$ and $\sigma^{2}$ can be estimated. 
For parameter $\delta_{i-1}=\mu_{i}-\mu_{i-1}(2<i<k)$, we can choose a prior distribution that is a mixture of a Normal distribution and a discrete distribution with its entire mass at $\delta_{i}=0$. Meanwhile, a latent variable $\gamma_{i}$ is used to model the difference status of the two means, and it is expressed as:

$$
\begin{cases}\delta_{i}=0, & \text { if } \gamma_{i}=0 \\ \delta_{i} \sim \mathrm{N}\left(0, \sigma_{\Delta}^{2}\right), & \text { if } \gamma_{i}=1\end{cases}
$$

Thus, $\delta_{i}$ is modeled by a mixture of two components, one is being a point mass for $\mu_{i}=\mu_{i-1}$, i.e. $\delta_{i}=0$; and another is being a normal distribution for the inequality of two means, $\mu_{i} \neq \mu_{i-1}$, i.e. $\delta_{i} \neq 0$. Note that $\gamma_{i}=0 / 1$ indicates that the two means are equal/unequal, and it is modeled by a Bernoulli distribution, $\gamma_{i} \mid B_{r} \sim \operatorname{Bernoulli}(\operatorname{Br})$, where $P_{r}$ is the probability for the case $\gamma_{i}=1$.

With $I_{(A)}$ denoting the indicator function corresponding to an event $A$, the density function for $\delta_{i}$ can be represented as

$$
\left[\delta_{i} \mid P_{r}, \sigma_{\Delta}^{2}\right]=\left(1-P_{r}\right) I_{\left(\delta_{i}=0\right)}+P_{r} \Delta I_{\left(\delta_{i} \neq 0\right)}
$$

where $\Delta=\frac{1}{\sqrt{2 \pi} \sigma_{\Delta}} \exp \left\{-\frac{\delta_{i}^{2}}{2 \sigma_{\Delta}^{2}}\right\}$.

Next, we specify the priors for hyper-parameters $\sigma_{\Delta}^{2}$ and $P_{r}$.

Let $\sigma_{\Delta}^{2} \sim \operatorname{IG}\left(a_{0}, b_{0}\right)$, here $\operatorname{IG}\left(a_{0}, b_{0}\right)$ denotes an inverse gamma distribution with den- 
sity function

$$
\left[\sigma_{\Delta}^{2} \mid a_{o}, b_{o}\right]=\frac{1}{\Gamma\left(a_{0}\right)\left(b_{0}\right)^{a_{0}}} \frac{1}{\left(\sigma_{\Delta}^{2}\right)^{a_{0}+1}} \exp \left(-\frac{1}{b_{0} \sigma_{\Delta}^{2}}\right)
$$

And $P_{r} \sim \operatorname{Beta}\left(a_{r}, b_{r}\right)$, where $a_{r}$ and $b_{r}$ are provided, and we intend to let $P_{r}$ as non-informative as possible.

In what follows, we will conduct the multiple comparisons by the proposed Bayesian Hierarchical Model. More specifically, our interest is $E\left(\gamma_{i} \mid Y\right)$ or $1-E\left(\gamma_{i} \mid Y\right)$, the marginal posterior probabilities that the true means and not equal or equal, respectively.

\subsection{Full Conditional Distributions}

\subsubsection{Full Conditional Posterior Distribution of $\mu_{1}$}

Let $Y=\left(y_{1}^{\prime}, \ldots, y_{m}^{\prime}\right)^{\prime}$. Given that the cases are independent, the joint density function of the data vector $\mathrm{Y}$ is given by Equation (4.4) and the prior given by Equation (4.5)

Then the full conditional posterior distribution of $\mu_{1}$ is

$$
\left[\mu_{1} \mid Y, \sigma^{2}, \sigma_{\tau}^{2},\left\{\delta_{i}\right\}\right]=\mathrm{N}\left(\frac{p}{q}, \frac{1}{q}\right)
$$

where $p=\frac{\mu_{0}}{\tau_{0}^{2}}+\frac{s_{3}}{k \sigma_{\tau}^{2}+\sigma^{2}}$, and $q=\frac{1}{\tau_{0}^{2}}+\frac{k m}{k \sigma_{\tau}^{2}+\sigma^{2}}, s_{3}=\sum_{j=1}^{m} \sum_{i=1}^{k}\left(y_{i j}-\sum_{l=0}^{i-1} \delta_{l}\right)$.

From the joint density function of the data vector $Y$ given by (4.4), we have:

$$
s_{1}=\sum_{j=1}^{m} \sum_{i=1}^{k}\left(y_{i j}-\mu_{1}-\sum_{l=0}^{i-1} \delta_{l}\right)^{2} \text { and } s_{2}=\left(\sum_{j=1}^{m} \sum_{i=1}^{k}\left(y_{i j}-\mu_{1}-\sum_{l=0}^{i-1} \delta_{l}\right)\right)^{2} \text {. }
$$


In terms of the joint density function of $\mu_{1}$, we re-express $s_{1}$ and $s_{2}$ :

$$
\begin{aligned}
s_{1}= & \sum_{j=1}^{m} \sum_{i=1}^{k}\left(y_{i j}-\mu_{1}-\sum_{l=0}^{i-1} \delta_{l}\right)^{2}=\sum_{j=1}^{m} \sum_{i=1}^{k}\left(\mu_{1}-\left(y_{i j}-\sum_{l=0}^{i-1} \delta_{l}\right)\right)^{2} \\
& \propto \sum_{j=1}^{m} \sum_{i=1}^{k}\left(\mu_{1}^{2}-2 \mu_{1}\left(y_{i j}-\sum_{l=0}^{i-1} \delta_{l}\right)\right)=k m \mu_{1}^{2}-2 \mu_{1} \sum_{j=1}^{m} \sum_{i=1}^{k}\left(y_{i j}-\sum_{l=0}^{i-1} \delta_{l}\right) . \\
s_{2}= & \sum_{j=1}^{m}\left(\sum_{i=1}^{k}\left(y_{i j}-\mu_{1}-\sum_{l=0}^{i-1} \delta_{l}\right)\right)^{2}=\sum_{j=1}^{m}\left(k \mu_{1}-\sum_{i=1}^{k}\left(y_{i j}-\sum_{l=0}^{i-1} \delta_{l}\right)\right)^{2} \\
& \propto \sum_{j=1}^{m}\left(k^{2} \mu_{1}^{2}-2 k \mu_{1}\left(\sum_{i=1}^{k}\left(y_{i j}-\sum_{l=0}^{i-1} \delta_{l}\right)\right)\right)=m k^{2} \mu_{1}^{2}-2 k \mu_{1} \sum_{j=1}^{m} \sum_{i=1}^{k}\left(y_{i j}-\sum_{l=0}^{i-1} \delta_{l}\right) .
\end{aligned}
$$

Let $s_{3}=\sum_{j=1}^{m} \sum_{i=1}^{k}\left(y_{i j}-\sum_{l=0}^{i-1} \delta_{l}\right)$, we can re-write the expression as $s_{1}=k m \mu_{1}^{2}-2 \mu_{1} s_{3}$ and $s_{2}=m k^{2} \mu_{1}^{2}-2 k \mu_{1} s_{3}$.

And $\mu_{1} \sim \mathrm{N}\left(\mu_{0}, \tau_{0}^{2}\right)$, where $f\left(\mu_{1}\right)=\frac{1}{\tau_{0}} \exp \left(-\frac{\left(\mu_{1}-\mu_{0}\right)^{2}}{2 \tau_{0}^{2}}\right)$, then conditional posterior distribution of $\mu_{1}$ can be written as

$$
\begin{aligned}
& {\left[\mu_{1} \mid Y, \sigma^{2}, \sigma_{\tau}^{2},\left\{\delta_{i}\right\}\right]=\left[Y \mid \mu_{1}, \sigma^{2}, \sigma_{\tau}^{2},\left\{\delta_{i}\right\}\right] \times P\left(\mu_{1}\right)} \\
& \quad \propto \exp \left\{-\frac{1}{2 \sigma^{2}}\left[\left(m k \mu_{1}^{2}-2 \mu_{1} s_{3}\right)-\frac{\sigma_{\tau}^{2}}{k \sigma_{\tau}^{2}+\sigma^{2}}\left(m k^{2} \mu_{1}^{2}-2 k \mu_{1} s_{3}\right)\right]\right\} \times P\left(\mu_{1}\right) \\
& \quad \propto \exp \left\{-\frac{m k \mu_{1}^{2}-2 \mu_{1} s_{3}}{2 \sigma^{2}}+\frac{\sigma_{\tau}^{2}\left(m k^{2} \mu_{1}^{2}-2 k \mu_{1} s_{3}\right)}{2 \sigma^{2}\left(k \sigma_{\tau}^{2}+\sigma^{2}\right)}\right\} \times \exp \left\{-\frac{\mu_{1}^{2}-2 \mu_{1} \mu_{0}}{2 \tau_{0}^{2}}\right\}
\end{aligned}
$$




$$
\begin{aligned}
& =\exp \left\{-\frac{m k \mu_{1}^{2}}{2 \sigma^{2}}+\frac{2 \mu_{1} s_{3}}{2 \sigma^{2}}+\frac{m k^{2} \mu_{1}^{2} \sigma_{\tau}^{2}}{2 \sigma^{2}\left(k \sigma_{\tau}^{2}+\sigma^{2}\right)}-\frac{2 k \mu_{1} s_{3} \sigma_{\tau}^{2}}{2 \sigma^{2}\left(k \sigma_{\tau}^{2}+\sigma^{2}\right)}-\frac{\mu_{1}^{2}}{2 \tau_{0}^{2}}+\frac{2 \mu_{1} \mu_{0}}{2 \tau_{0}^{2}}\right\} \\
& =\exp \left\{-\frac{\mu_{1}^{2}}{2}\left(\frac{m k}{\sigma^{2}}-\frac{m k^{2} \sigma_{\tau}^{2}}{\sigma^{2}\left(k \sigma_{\tau}^{2}+\sigma^{2}\right)}+\frac{1}{\tau_{0}^{2}}\right)+\mu_{1}\left(\frac{s_{3}}{\sigma^{2}}+\frac{\mu_{0}}{2 \tau_{0}^{2}}-\frac{2 k s_{3} \sigma_{\tau}^{2}}{\sigma^{2}\left(k \sigma_{\tau}^{2}+\sigma^{2}\right)}\right)\right\} \\
& =\exp \left\{-\frac{\mu_{1}^{2}}{2}\left(\frac{1}{\tau_{0}^{2}}+\frac{m k}{k \sigma_{\tau}^{2}+\sigma^{2}}\right)+\mu_{1}\left(\frac{\mu_{0}}{2 \tau_{0}^{2}}+\frac{s_{3}}{k \sigma_{\tau}^{2}+\sigma^{2}}\right)\right\} .
\end{aligned}
$$

Let $p=\frac{\mu_{0}}{2 \tau_{0}^{2}}+\frac{s_{3}}{k \sigma_{\tau}^{2}+\sigma^{2}}$ and $q=\frac{1}{\tau_{0}^{2}}+\frac{m k}{k \sigma_{\tau}^{2}+\sigma^{2}}$, then we have

$$
\begin{aligned}
& =\exp \left\{-\frac{\mu_{1}^{2}}{2} \cdot q+\mu_{1} \cdot p\right\} \\
& =\exp \left\{-\frac{\mu_{1}^{2} \cdot q-2 \mu_{1} \cdot p}{2}\right\} \\
& =\exp \left\{-\frac{q\left(\mu_{1}^{2}-2 \cdot \frac{p}{q} \cdot \mu_{1}\right)}{2}\right\} \\
& =\exp \left\{-\frac{q\left(\mu_{1}^{2}-2 \cdot \frac{p}{q} \cdot \mu_{1}+\frac{p^{2}}{q^{2}}\right)-\frac{p^{2}}{q}}{2}\right\} \\
& \propto \exp \left\{-\frac{\left(\mu_{1}-\frac{p}{q}\right)^{2}}{2 \cdot \frac{1}{q}}\right\} .
\end{aligned}
$$

Thus, $\left[\mu_{1} \mid Y, \sigma^{2}, \sigma_{\tau}^{2},\left\{\delta_{i}\right\}\right]=\mathrm{N}\left(\frac{p}{q}, \frac{1}{q}\right)$, where $p=\frac{\mu_{0}}{2 \tau_{0}^{2}}+\frac{s_{3}}{k \sigma_{\tau}^{2}+\sigma^{2}}$ and $q=\frac{1}{\tau_{0}^{2}}+$ $\frac{m k}{k \sigma_{\tau}^{2}+\sigma^{2}}$ 


\subsubsection{Full Conditional Posterior Distribution of $\sigma^{2}$ and $\tau^{2}$}

Let $Y=\left(y_{1}^{\prime}, \ldots, y_{m}^{\prime}\right)^{\prime}$. Given that the cases are independent, the joint density function of the data vector $\mathrm{Y}$ is given by Equation (4.4) and the prior given by Equation (4.6)

We set $\tau^{2}=k \sigma_{\tau}^{2}+\sigma^{2}$, then the full conditional posterior distribution of $\sigma^{2}$ is

$$
\left[\sigma^{2} \mid Y, \mu_{1}, \sigma_{\tau}^{2},\left\{\delta_{i}\right\}\right]=\operatorname{IG}\left(\frac{(k-1) m}{2}, \frac{2}{\left(s_{1}-\frac{1}{k} s_{2}\right)}\right)
$$

and the full conditional posterior distribution of $\tau^{2}=k \sigma_{\tau}^{2}+\sigma^{2}$ is

$$
\begin{gathered}
{\left[\tau^{2} \mid Y, \mu_{1}, \sigma^{2},\left\{\delta_{i}\right\}\right]=\operatorname{IG}\left(\frac{m}{2}, \frac{2 k}{s_{2}}\right),} \\
\text { where } s_{1}=\sum_{j=1}^{m} \sum_{i=1}^{k}\left(y_{i j}-\mu_{1}-\sum_{l=0}^{i-1} \delta_{l}\right)^{2}, s_{2}=\sum_{j=1}^{m}\left(\sum_{i=1}^{k}\left(y_{i j}-\mu_{1}-\sum_{l=0}^{i-1} \delta_{l}\right)\right)^{2} .
\end{gathered}
$$

In Section 4.4, we set $\tau^{2}=k \sigma_{\tau}^{2}+\sigma^{2}$ and the prior $\pi\left(\tau^{2}, \sigma^{2}\right) \propto \frac{1}{\sigma^{2} \tau^{2}}$, thus we have

$$
\begin{aligned}
& {\left[Y \mid \mu_{1},\left\{\delta_{i}\right\}, \sigma_{\tau}^{2}, \sigma^{2}\right] \times P\left(\tau^{2}, \sigma^{2}\right)} \\
& \quad \propto\left(\sigma^{2}\right)^{-\frac{m(k-1)}{2}}\left(k \sigma_{\tau}^{2}+\sigma^{2}\right)^{-\frac{m}{2}} \exp \left\{-\frac{1}{2 \sigma^{2}}\left[s_{1}-\frac{\sigma_{\tau}^{2}}{k \sigma_{\tau}^{2}+\sigma^{2}} s_{2}\right]\right\} \times \frac{1}{\sigma^{2} \tau^{2}} \\
& \quad=\left(\sigma^{2}\right)^{-\frac{m(k-1)}{2}}\left(\tau^{2}\right)^{-\frac{m}{2}} \exp \left\{-\frac{1}{2 \sigma^{2}}\left[s_{1}-\frac{\sigma_{\tau}^{2}}{\tau^{2}} s_{2}\right]\right\} \times \frac{1}{\sigma^{2} \tau^{2}}
\end{aligned}
$$




$$
\begin{aligned}
& =\left(\sigma^{2}\right)^{-\frac{m(k-1)}{2}-1}\left(\tau^{2}\right)^{-\frac{m}{2}-1} \exp \left\{-\frac{1}{2 \sigma^{2}} s_{1}+\frac{\sigma_{\tau}^{2}}{2 \sigma^{2} \tau^{2}} s_{2}\right\} \\
& =\left(\sigma^{2}\right)^{-\frac{m(k-1)}{2}-1}\left(\tau^{2}\right)^{-\frac{m}{2}-1} \exp \left\{-\frac{1}{2 \sigma^{2}} s_{1}+\frac{\tau^{2}-\sigma^{2}}{k 2 \sigma^{2} \tau^{2}} s_{2}\right\}, \text { where } \sigma_{\tau}^{2}=\tau^{2}-\sigma^{2} \\
& =\left(\sigma^{2}\right)^{-\frac{m(k-1)}{2}-1}\left(\tau^{2}\right)^{-\frac{m}{2}-1} \exp \left\{-\frac{1}{2 \sigma^{2}} s_{1}+\frac{\tau^{2}}{k 2 \sigma^{2} \tau^{2}} s_{2}-\frac{\sigma^{2}}{k 2 \sigma^{2} \tau^{2}} s_{2}\right\} \\
& =\left(\sigma^{2}\right)^{-\frac{m(k-1)}{2}-1}\left(\tau^{2}\right)^{-\frac{m}{2}-1} \exp \left\{-\frac{1}{2 \sigma^{2}} s_{1}+\frac{1}{2 k \sigma^{2}} s_{2}-\frac{1}{2 k \tau^{2}} s_{2}\right\} \\
& =\left(\sigma^{2}\right)^{-\frac{m(k-1)}{2}-1}\left(\tau^{2}\right)^{-\frac{m}{2}-1} \exp \left\{-\frac{1}{2 \sigma^{2}}\left(s_{1}-\frac{1}{k} s_{2}\right)-\frac{1}{2 k \tau^{2}} s_{2}\right\} \\
& =\left(\sigma^{2}\right)^{-\frac{m(k-1)}{2}-1} \exp \left\{-\frac{1}{2 \sigma^{2}}\left(s_{1}-\frac{1}{k} s_{2}\right)\right\} \times\left(\tau^{2}\right)^{-\frac{m}{2}-1} \exp \left\{-\frac{1}{2 k \tau^{2}} s_{2}\right\} .
\end{aligned}
$$

Thus, we have the full conditional posterior distribution of $\sigma^{2}$ as

$$
\begin{aligned}
{\left[\sigma^{2} \mid Y, \mu_{1}, \sigma_{\tau}^{2},\left\{\delta_{i}\right\}\right] } & =\left(\sigma^{2}\right)^{-\frac{m(k-1)}{2}-1} \exp \left\{-\frac{1}{2 \sigma^{2}}\left(s_{1}-\frac{1}{k} s_{2}\right)\right\} \\
& =\frac{1}{\left(\sigma^{2}\right)^{\frac{m(k-1)}{2}+1}} \exp \left\{-\frac{1}{\left(\frac{2}{s_{1}-\frac{1}{k} s_{2}}\right) \sigma^{2}}\right\} \\
& \propto \operatorname{IG}\left(\frac{m(k-1)}{2}, \frac{2}{s_{1}-\frac{1}{k} s_{2}}\right) .
\end{aligned}
$$

The full conditional posterior distribution of $\tau^{2}$ as 


$$
\begin{aligned}
{\left[\tau^{2} \mid Y, \mu_{1}, \sigma^{2},\left\{\delta_{i}\right\}\right] } & =\left(\tau^{2}\right)^{-\frac{m}{2}-1} \exp \left\{-\frac{1}{2 k \tau^{2}} s_{2}\right\} \\
& =\frac{1}{\left(\tau^{2}\right)^{\frac{m}{2}+1}} \exp \left\{-\frac{1}{\left(\frac{2 k}{s_{2}}\right) \tau^{2}}\right\} \\
& \propto \operatorname{IG}\left(\frac{m}{2}, \frac{2 k}{s_{2}}\right) .
\end{aligned}
$$

Note, we use the form of the inverse gamma distribution $\operatorname{IG}(\alpha, \beta)$ with the density function as

$$
f(x)=\frac{1}{\Gamma(\alpha) \beta^{\alpha}} \cdot \frac{\exp \left\{-\frac{1}{\beta x}\right\}}{x^{\alpha+1}} .
$$

\subsubsection{Full Conditional Posterior Distribution of $\delta_{i}$}

Let $Y=\left(y_{1}^{\prime}, \ldots, y_{m}^{\prime}\right)^{\prime}$. Given that the cases are independent, the joint density function of the data vector $Y$ is given by Equation (4.4), and the prior for $\delta_{i}$ is $\left[\delta_{i} \mid P_{r}, \sigma_{\Delta}^{2}\right]=$ $\left(1-P_{r}\right) I_{\left(\delta_{i}=0\right)}+P_{r} \Delta I_{\left(\delta_{i} \neq 0\right)}$, where $\Delta \sim \mathrm{N}\left(0, \sigma_{\Delta}^{2}\right)$

Then the full conditional posterior distribution of $\delta_{i}$ is a mixture of a discrete part and a continuous part, and it is written as

$$
\left[\delta_{i} \mid \bullet\right]= \begin{cases}c_{i}\left(1-P_{r}\right) h\left(\delta_{i}\right), & \delta_{i}=0 . \\ c_{i} P_{r} \frac{1}{\sigma_{\Delta}} h\left(\delta_{i}\right), & \delta_{i} \neq 0 .\end{cases}
$$

where, 


$$
\begin{aligned}
& c_{i}=\frac{1}{\left(1-P_{r}\right) h(0)+P_{r} \frac{1}{\sigma_{\Delta}} \int_{0}^{\infty} h\left(\delta_{i}\right) d \delta_{i}}, \\
& h\left(\delta_{i}\right)=\frac{1}{\sqrt{2 \pi\left(\frac{1}{a_{i}}\right)}} \exp \left\{-\frac{1}{2\left(\frac{1}{a_{i}}\right)}\left(\delta_{i}-\frac{g_{i}}{a_{i}}\right)^{2}\right\} \exp \left\{\frac{g_{i}^{2}}{2 a_{i}}\right\}, \text { with } \\
& a_{i}=\frac{m}{\sigma^{2}}\left((k-i)-\frac{\sigma_{\tau}^{2}(k-i)^{2}}{k \sigma_{\tau}^{2}+\sigma^{2}}\right)+\frac{\Delta_{i}}{\sigma_{\Delta}^{2}} \\
& \Delta_{i}=I_{\left(\delta_{i} \neq 0\right)}, \\
& g_{i}=\frac{1}{\sigma^{2}}\left(1-\frac{\sigma_{\tau}^{2}(k-i)}{k \sigma_{\tau}^{2}+\sigma^{2}}\right) s_{i}, \text { and } \\
& s_{i}=\sum_{j=1}^{m} \sum_{p=i+1}^{k-1}\left(y_{p j}-\mu_{1}-\sum_{q=1} \delta_{q}+\delta_{i}\right) .
\end{aligned}
$$

From the joint density function of the data vector Y given by Equation (4.4), we have:

$$
s_{1}=\sum_{j=1}^{m} \sum_{i=1}^{k}\left(y_{i j}-\mu_{1}-\sum_{l=0}^{i-1} \delta_{l}\right)^{2}, \text { and } s_{2}=\sum_{j=1}^{m}\left(\sum_{i=1}^{k}\left(y_{i j}-\mu_{1}-\sum_{l=0}^{i-1} \delta_{l}\right)\right)^{2} \text {. }
$$

In the posterior distribution of $\delta_{i}$, we re-express $s_{1}$ and $s_{2}$ :

$$
\begin{aligned}
s_{1}= & \sum_{j=1}^{m} \sum_{i=1}^{k}\left(y_{i j}-\mu_{1}-\sum_{l=0}^{i-1} \delta_{l}\right)^{2}=\sum_{j=1}^{m} \sum_{p=i+1}^{k}\left(y_{p j}-\mu_{1}-\sum_{q=1}^{p-1} \delta_{q}\right)^{2} \\
& =\sum_{j=1}^{m} \sum_{p=i+1}^{k}\left(\delta_{i}-\left(y_{p j}-\mu_{1}-\sum_{q=1}^{p-1} \delta_{q}+\delta_{i}\right)\right)^{2} \\
& \propto m(k-i) \delta_{i}^{2}-2 \delta_{i}\left(\sum_{j=1}^{m} \sum_{p=i+1}^{k}\left(y_{p j}-\mu_{1}-\sum_{q=1}^{p-1} \delta_{q}+\delta_{i}\right)\right) \\
& =m(k-i) \delta_{i}^{2}-2 \delta_{i} s_{i}, \text { where } s_{i}=\sum_{j=1}^{m} \sum_{p=i+1}^{k}\left(y_{p j}-\mu_{1}-\sum_{q=0}^{p-1} \delta_{q}+\delta_{i}\right),
\end{aligned}
$$




$$
\begin{aligned}
s_{2}= & \sum_{j=1}^{m}\left(\sum_{i=1}^{k}\left(y_{i j}-\mu_{1}-\sum_{l=0}^{i-1} \delta_{l}\right)\right)^{2}=\sum_{j=1}^{m}\left(\sum_{p=i+1}^{k}\left(y_{p j}-\mu_{1}-\sum_{q=1}^{p-1} \delta_{q}\right)\right)^{2} \\
& =\sum_{j=1}^{m}\left(\sum_{p=i+1}^{k}\left(\delta_{i}-\left(y_{p j}-\mu_{1}-\sum_{q=1}^{p-1} \delta_{q}+\delta_{i}\right)\right)^{2}\right. \\
& =\sum_{j=1}^{m}\left((k-i) \delta_{i}-\sum_{p=i+1}^{k}\left(y_{p j}-\mu_{1}-\sum_{q=1}^{p-1} \delta_{q}+\delta_{i}\right)\right)^{2} \\
& \propto \sum_{j=1}^{m}\left((k-i)^{2} \delta_{i}^{2}-2(k-i) \delta_{i} \sum_{p=i+1}^{k}\left(y_{p j}-\mu_{1}-\sum_{q=1}^{p-1} \delta_{q}+\delta_{i}\right)\right) \\
& =m(k-i)^{2} \delta_{i}^{2}-2(k-i) \delta_{i} \sum_{j=1}^{m} \sum_{p=i+1}^{k}\left(y_{p j}-\mu_{1}-\sum_{q=1}^{p-1} \delta_{q}+\delta_{i}\right) \\
& =m(k-i)^{2} \delta_{i}^{2}-2(k-i) \delta_{i} s_{i}, \text { where } s_{i}=\sum_{j=1}^{m} \sum_{p=i+1}^{k}\left(y_{p j}-\mu_{1}-\sum_{q=0}^{p-1} \delta_{q}+\delta_{i}\right) .
\end{aligned}
$$

Thus, the posterior distribution of $\delta_{i}$ can be expressed by the product of the joint density function given by (4.4) and the prior of $\delta_{i}$, which is given by $\left[\delta_{i} \mid P, \sigma_{\Delta}^{2}\right]=\left(1-P_{r}\right) I_{\left(\delta_{i}=0\right)}+$ $P_{r} \Delta I_{\left(\delta_{i} \neq 0\right)}$, where $\Delta \sim \mathrm{N}\left(0, \sigma_{\Delta}^{2}\right)$,

For $i=1, \ldots, k-1$, we have

$$
\begin{aligned}
& {\left[\delta_{i} \mid Y, \mu_{1}, \sigma_{\tau}^{2}, \sigma^{2},\left\{\delta_{1}, \ldots, \delta_{i-1}, \delta_{i+1}, \ldots, \delta_{k-1}\right\}, P_{r}, \sigma_{\Delta}^{2}\right]} \\
& =\left(\sigma^{2}\right)^{-\frac{m(k-1)}{2}}\left(k \sigma_{\tau}^{2}+\sigma^{2}\right)^{-\frac{m}{2}} \exp \left\{-\frac{1}{2 \sigma^{2}}\left[s_{1}-\frac{\sigma_{\tau}^{2}}{k \sigma_{\tau}^{2}+\sigma^{2}} s_{2}\right]\right\} *\left(\left(1-P_{r}\right) I_{\left(\delta_{i}=0\right)}+P_{r} \Delta I_{\left(\delta_{i} \neq 0\right)}\right) \\
& \propto \exp \left\{-\frac{1}{2 \sigma^{2}}\left[s_{1}-\frac{\sigma_{\tau}^{2}}{k \sigma_{\tau}^{2}+\sigma^{2}} s_{2}\right]\right\} *\left(\left(1-P_{r}\right) I_{\left(\delta_{i}=0\right)}+P_{r} \Delta I_{\left(\delta_{i} \neq 0\right)}\right)
\end{aligned}
$$




$$
\begin{aligned}
& =\exp \left\{-\frac{1}{2 \sigma^{2}}\left[m(k-i) \delta_{i}^{2}-2 \delta_{i} s_{i}-\frac{\sigma_{\tau}^{2}}{k \sigma_{\tau}^{2}+\sigma^{2}}\left(m(k-i)^{2} \delta_{i}^{2}-2(k-i) \delta_{i} s_{i}\right)\right]\right\} *\left(\left(1-P_{r}\right) I_{\left(\delta_{i}=0\right)}+\right. \\
& \left.P_{r} \Delta I_{\left(\delta_{i} \neq 0\right)}\right) \\
& =\exp \left\{-\frac{1}{2 \sigma^{2}}\left[m(k-i)\left(1-\frac{\sigma_{\tau}^{2}(k-i)}{k \sigma_{\tau}^{2}+\sigma^{2}}\right) \delta_{i}^{2}-2 s_{i}\left(1-\frac{\sigma_{\tau}^{2}(k-i)}{k \sigma_{\tau}^{2}+\sigma^{2}}\right) \delta_{i}\right]\right\} *\left(\left(1-P_{r}\right) I_{\left(\delta_{i}=0\right)}+\right. \\
& \left.P_{r} \Delta I_{\left(\delta_{i} \neq 0\right)}\right) \\
& =\exp \left\{-\frac{1}{2 \sigma^{2}}\left[m\left((k-i)-\frac{\sigma_{\tau}^{2}(k-i)^{2}}{k \sigma_{\tau}^{2}+\sigma^{2}}\right) \delta_{i}^{2}-2 s_{i}\left(1-\frac{\sigma_{\tau}^{2}(k-i)}{k \sigma_{\tau}^{2}+\sigma^{2}}\right) \delta_{i}\right]\right\} *\left(1-P_{r}\right) I_{\left(\delta_{i}=0\right)}+ \\
& \exp \left\{-\frac{1}{2 \sigma^{2}}\left[m\left((k-i)-\frac{\sigma_{\tau}^{2}(k-i)^{2}}{k \sigma_{\tau}^{2}+\sigma^{2}}\right) \delta_{i}^{2}-2 s_{i}\left(1-\frac{\sigma_{\tau}^{2}(k-i)}{k \sigma_{\tau}^{2}+\sigma^{2}}\right) \delta_{i}\right]\right\} * P_{r}\left(\frac{1}{\sqrt{2 \pi} \sigma_{\Delta}} \exp \left\{-\frac{\delta_{i}^{2}}{2 \sigma_{\Delta}^{2}}\right\}\right) I_{\left(\delta_{i} \neq 0\right)} .
\end{aligned}
$$

Thus, in order to find the conditional posterior distribution of $\delta_{i}$, we first look at the conditional posterior distribution when $\delta_{i} \neq 0$,

$\left[\delta_{i} \mid Y, \mu_{1}, \sigma_{\tau}^{2}, \sigma^{2},\left\{\delta_{1}, \ldots, \delta_{i-1}, \delta_{i+1}, \ldots, \delta_{k-1}\right\}, P_{r}, \sigma_{\Delta}^{2}\right]$

$$
\begin{aligned}
& =\exp \left\{-\frac{1}{2 \sigma^{2}}\left[m\left((k-i)-\frac{\sigma_{\tau}^{2}(k-i)^{2}}{k \sigma_{\tau}^{2}+\sigma^{2}}\right) \delta_{i}^{2}-2 s_{i}\left(1-\frac{\sigma_{\tau}^{2}(k-i)}{k \sigma_{\tau}^{2}+\sigma^{2}}\right) \delta_{i}\right]\right\} * P_{r} \frac{1}{\sqrt{2 \pi} \sigma_{\Delta}} \exp \left\{-\frac{\delta_{i}^{2}}{2 \sigma_{\Delta}^{2}}\right\} \\
& =\frac{P_{r}}{\sqrt{2 \pi} \sigma_{\Delta}} \exp \left\{-\frac{1}{2}\left[\frac{m}{\sigma^{2}}\left((k-i)-\frac{\sigma_{\tau}^{2}(k-i)^{2}}{k \sigma_{\tau}^{2}+\sigma^{2}}\right) \delta_{i}^{2}-2 \frac{s_{i}}{\sigma^{2}}\left(1-\frac{\sigma_{\tau}^{2}(k-i)}{k \sigma_{\tau}^{2}+\sigma^{2}}\right) \delta_{i}\right]-\frac{\delta_{i}^{2}}{2 \sigma_{\Delta}^{2}}\right\} \\
& =\frac{P_{r}}{\sqrt{2 \pi} \sigma_{\Delta}} \exp \left\{-\frac{1}{2}\left[\frac{m}{\sigma^{2}}\left((k-i)-\frac{\sigma_{\tau}^{2}(k-i)^{2}}{k \sigma_{\tau}^{2}+\sigma^{2}}\right) \delta_{i}^{2}+\frac{1}{\sigma_{\Delta}^{2}} \delta_{i}^{2}-2 \frac{s_{i}}{\sigma^{2}}\left(1-\frac{\sigma_{\tau}^{2}(k-i)}{k \sigma_{\tau}^{2}+\sigma^{2}}\right) \delta_{i}\right]\right\}
\end{aligned}
$$




$$
\begin{aligned}
& =\frac{P_{r}}{\sqrt{2 \pi} \sigma_{\Delta}} \exp \left\{-\frac{1}{2}\left[\left(\frac{m}{\sigma^{2}}\left((k-i)-\frac{\sigma_{\tau}^{2}(k-i)^{2}}{k \sigma_{\tau}^{2}+\sigma^{2}}\right)+\frac{1}{\sigma_{\Delta}^{2}}\right) \delta_{i}^{2}-2 \frac{s_{i}}{\sigma^{2}}\left(1-\frac{\sigma_{\tau}^{2}(k-i)}{k \sigma_{\tau}^{2}+\sigma^{2}}\right) \delta_{i}\right]\right\} \\
& =\frac{P_{r}}{\sqrt{2 \pi} \sigma_{\Delta}} \exp \left\{-\frac{1}{2}\left[a \delta_{i}^{2}-2 b \delta_{i}\right]\right\} \\
& =\frac{P_{r}}{\sqrt{2 \pi} \sigma_{\Delta}} \exp \left\{-\frac{1}{2}\left[a\left(\delta_{i}^{2}-2 \frac{b}{a} \delta_{i}\right)\right]\right\} \\
& =\frac{P_{r}}{\sqrt{2 \pi} \sigma_{\Delta}} \exp \left\{-\frac{1}{2}\left[a\left(\delta_{i}^{2}-2 \frac{b}{a} \delta_{i}+\frac{b^{2}}{a^{2}}-\frac{b^{2}}{a^{2}}\right)\right]\right\} \\
& =\frac{P_{r}}{\sqrt{2 \pi} \sigma_{\Delta}} \exp \left\{-\frac{1}{2}\left[a\left(\left(\delta_{i}-\frac{b}{a}\right)^{2}-\frac{b^{2}}{a^{2}}\right)\right]\right\} \\
& \left.=\frac{P_{r}}{\sqrt{2 \pi} \sigma_{\Delta}} \exp \left\{-\frac{1}{2}\left[a\left(\delta_{i}-\frac{b}{a}\right)^{2}-\frac{b^{2}}{a}\right)\right]\right\} \\
& =\frac{P_{r}}{\sqrt{2 \pi} \sigma_{\Delta}} \exp \left\{-\frac{1}{2}\left[a\left(\delta_{i}-\frac{b}{a}\right)^{2}\right]\right\} \exp \left\{\frac{b^{2}}{2 a}\right\} \\
& \propto P_{r} \frac{1}{\sigma_{\Delta}} \frac{1}{\sqrt{2 \pi}(1 / a)} \exp \left\{-\frac{1}{2(1 / a)}\left[\left(\delta_{i}-\frac{b}{a}\right)^{2}\right]\right\} \exp \left\{\frac{b^{2}}{2 a}\right\} \\
& \text { where } a=\frac{m}{\sigma^{2}}\left((k-i)-\frac{\sigma_{\tau}^{2}(k-i)^{2}}{k \sigma_{\tau}^{2}+\sigma^{2}}\right)+\frac{1}{\sigma_{\Delta}^{2}} \text {, and } b=\frac{s_{i}}{\sigma^{2}}\left(1-\frac{\sigma_{\tau}^{2}(k-i)}{k \sigma_{\tau}^{2}+\sigma^{2}}\right) \text {. }
\end{aligned}
$$

If $\delta_{1}=0$, the prior probability of $\delta_{1}$ is $\left(1-P_{r}\right)$ and $\sigma_{\Delta}^{2}$ drops out of the last terms in the last product. 
Therefore, the full conditional posterior distribution of $\delta_{i}$ can be summarized as a mixture of a discrete part and a continuous part.

$$
\left[\delta_{i} \mid \bullet\right]= \begin{cases}c_{i}\left(1-P_{r}\right) h\left(\delta_{i}\right), & \delta_{i}=0 . \\ c_{i} P_{r} \frac{1}{\sigma_{\Delta}} h\left(\delta_{i}\right), & \delta_{i} \neq 0 .\end{cases}
$$

where,

$$
\begin{aligned}
& c=\frac{1}{\left(1-P_{r}\right) h(0)+P_{r} \frac{1}{\sigma_{\Delta}} \int_{0}^{\infty} h\left(\delta_{i}\right) d \delta_{i}}, \\
& h\left(\delta_{i}\right)=\frac{1}{\sqrt{2 \pi\left(\frac{1}{a_{i}}\right)}} \exp \left\{-\frac{1}{2\left(\frac{1}{a_{i}}\right)}\left(\delta_{i}-\frac{g_{i}}{a_{i}}\right)^{2}\right\} \exp \left\{\frac{g_{i}^{2}}{2 a_{i}}\right\}, \text { with } \\
& a_{i}=\frac{m}{\sigma^{2}}\left((k-i)-\frac{\sigma_{\tau}^{2}(k-i)^{2}}{k \sigma_{\tau}^{2}+\sigma^{2}}\right)+\frac{\Delta_{i}}{\sigma_{\Delta}^{2}} \\
& \Delta_{i}=I_{\left(\delta_{i} \neq 0\right)} \\
& g_{i}=\frac{1}{\sigma^{2}}\left(1-\frac{\sigma_{\tau}^{2}(k-i)}{k \sigma_{\tau}^{2}+\sigma^{2}}\right) s_{i}, \text { and } \\
& s_{i}=\sum_{j=1}^{m} \sum_{p=i+1}^{k}\left(y_{p j}-\mu_{1}-\sum_{q=1}^{p-1} \delta_{q}+\delta_{i}\right) .
\end{aligned}
$$

\subsubsection{Full Conditional Posterior Distribution of $P_{r}$}

From Section 4.4 the prior on $P_{r} \sim \operatorname{BETA}\left(\alpha_{r}, \beta_{r}\right)$, and the full conditional posterior distribution of $\delta_{i}$ in Equation (4.14), the full conditional posterior distribution of $P_{r}$ can be expressed as

$$
\left[P_{r} \mid \delta_{i}, \sigma_{\Delta}^{2}\right]= \begin{cases}\operatorname{BETA}\left(\alpha_{r}, \beta_{r}+1\right), & \delta_{i}=0 . \\ \operatorname{BETA}\left(\alpha_{r}+1, \beta_{r}\right), & \delta_{i} \neq 0\end{cases}
$$

With $\alpha_{r}=\beta_{r}=1$ (uniform), we have 


$$
\left[P_{r} \mid \delta_{i}, \sigma_{\Delta}^{2}\right]= \begin{cases}\operatorname{BETA}(1,2), & \delta_{i}=0 \\ \operatorname{BETA}(2,1), & \delta_{i} \neq 0\end{cases}
$$

Since the density function for $\delta_{i}$ can be represented as $\left[\delta_{i} \mid P_{r}, \sigma_{\Delta}^{2}\right]=\left(1-P_{r}\right) I_{\left(\delta_{i}=0\right)}+$ $P_{r} \Delta I_{\left(\delta_{i} \neq 0\right)}$, where $\Delta=\frac{1}{\sqrt{2 \pi} \sigma_{\Delta}} \exp \left\{-\frac{\delta_{i}^{2}}{2 \sigma_{\Delta}^{2}}\right\}$, and the prior on $P_{r} \sim \operatorname{BETA}\left(\alpha_{r}, \beta_{r}\right)$, thus we have

$$
\begin{aligned}
& {\left[P_{r} \mid \sigma_{\Delta}^{2}\right]= \begin{cases}\left(1-P_{r}\right) I_{\left(\delta_{i}=0\right)} \cdot \operatorname{BETA}\left(\alpha_{r}, \beta_{r}\right), & \delta_{i}=0 . \\
P_{r} \Delta I_{\left(\delta_{i} \neq 0\right)} \cdot \operatorname{BETA}\left(\alpha_{r}, \beta_{r}\right), & \delta_{i} \neq 0 .\end{cases} } \\
& \propto \begin{cases}\left(1-P_{r}\right) \cdot P_{r}^{\alpha_{r}}\left(1-P_{r}\right)^{\beta_{r}-1}, & \delta_{i}=0 . \\
P_{r} \cdot P_{r}^{\alpha_{r}}\left(1-P_{r}\right)^{\beta_{r}-1}, & \delta_{i} \neq 0 .\end{cases} \\
& = \begin{cases}P_{r}^{\alpha_{r}}\left(1-P_{r}\right)^{\left(\beta_{r}+1\right)-1}, & \delta_{i}=0 . \\
P_{r}^{\alpha_{r}+1}\left(1-P_{r}\right)^{\beta_{r}-1}, & \delta_{i} \neq 0 .\end{cases} \\
& \propto \begin{cases}\operatorname{BETA}\left(\alpha_{r}, \beta_{r}+1\right), & \delta_{i}=0 . \\
\operatorname{BETA}\left(\alpha_{r}+1, \beta_{r}\right), & \delta_{i} \neq 0 .\end{cases}
\end{aligned}
$$




\subsubsection{Full Conditional Posterior Distribution of $\sigma_{\Delta}^{2}$}

For the prior on $\sigma_{\Delta}^{2} \sim \operatorname{IG}\left(a_{0}, b_{0}\right)$ and the full conditional posterior distribution of $\delta_{i}$ in Equation (4.14), the full conditional distribution of $\sigma_{\Delta}^{2}$ is given by

$$
\left[\sigma_{\Delta}^{2} \mid \delta_{i}, P_{r}\right]= \begin{cases}\operatorname{IG}\left(a_{0}, b_{0}\right), & \delta_{i}=0 . \\ \operatorname{IG}\left(a_{0}+\frac{1}{2},\left(\frac{\delta_{i}^{2}}{2}+\frac{1}{b_{0}}\right)^{-1}\right), & \delta_{i} \neq 0 .\end{cases}
$$

From (4.14), we have the density function $\left[\delta_{i} \mid P_{r}, \sigma_{\Delta}^{2}\right]=\left(1-P_{r}\right) I_{\left(\delta_{i}=0\right)}+P_{r} \Delta I_{\left(\delta_{i} \neq 0\right)}$, where $\Delta=\frac{1}{\sqrt{2 \pi} \sigma_{\Delta}} \exp \left\{-\frac{\delta_{i}^{2}}{2 \sigma_{\Delta}^{2}}\right\}$, and from the prior of $\sigma_{\Delta}^{2} \sim \operatorname{IG}\left(a_{0}, b_{0}\right)$, with density $\left[\sigma_{\Delta}^{2} \mid a_{0}, b_{0}\right]=\frac{1}{\Gamma\left(a_{0}\right)\left(b_{0}\right)^{a_{0}}} \frac{1}{\left(\sigma_{\Delta}^{2}\right)^{a_{0}+1}} \exp \left(-\frac{1}{b_{0} \sigma_{\Delta}^{2}}\right)$, thus, for $\delta_{i} \neq 0$, the full conditional distribution of $\sigma_{\Delta}^{2}$ is given by

$$
\begin{aligned}
{\left[\sigma_{\Delta}^{2} \mid \delta_{i}, P_{r}\right] } & =P_{r} \Delta I_{\left(\delta_{i} \neq 0\right)} \cdot \operatorname{IG}\left(a_{0}, b_{0}\right) \\
& =P_{r} \cdot \frac{1}{\sqrt{2 \pi} \sigma_{\Delta}} \exp \left\{-\frac{\delta_{i}^{2}}{2 \sigma_{\Delta}^{2}}\right\} \cdot \frac{1}{\Gamma\left(a_{0}\right) b_{0}^{a_{0}}}\left(\sigma_{\Delta}^{2}\right)^{-a_{0}-1} \exp \left\{-\frac{1}{b_{0} \sigma_{\Delta}^{2}}\right\} \\
& \propto \frac{1}{\sigma_{\Delta}} \cdot\left(\sigma_{\Delta}^{2}\right)^{-a_{0}-1} \exp \left\{-\frac{\delta_{1}^{2}}{2 \sigma_{\Delta}^{2}}-\frac{1}{b_{0} \sigma_{\Delta}^{2}}\right\} \\
& =\left(\sigma_{\Delta}^{2}\right)^{-a_{0}-1-\frac{1}{2}} \exp \left\{-\frac{\left(\frac{\delta_{i}^{2}}{2}+\frac{1}{b_{0}}\right)}{\sigma_{\Delta}^{2}}\right\} \\
& =\left(\sigma_{\Delta}^{2}\right)^{-\left(a_{0}+\frac{1}{2}\right)-1} \exp \left\{-\frac{1}{\left(\frac{\delta_{i}^{2}}{2}+\frac{1}{b_{0}}\right)^{-1} \sigma_{\Delta}^{2}}\right\} \\
& \propto \operatorname{IG}\left(\left(a_{0}+\frac{1}{2}\right),\left(\frac{\delta_{i}^{2}}{2}+\frac{1}{b_{0}}\right)^{-1}\right)^{-1}
\end{aligned}
$$




\subsection{Gibbs Sampling Steps}

By the presented proposed hierarchical model in Equation (4.4) and the full conditional posterior distribution for all parameters in Section 4.3, thus the algorithm for the Gibbs sampler method is expressed as follows:

Step 1: Set the initial values for parameters $\mu_{1}^{(0)}, \sigma_{\tau}^{2(0)}, \sigma^{2(0)}, \delta_{i}^{(0)}, P_{r}^{(0)}$ and $\sigma_{\triangle}^{2(0)}$ at time $t=0$.

Step 2: For each iteration, indexed $t=1,2, \ldots$ repeat:

(a) Set $\mu_{1}=\mu_{1}^{(t-1)}, \sigma_{\tau}^{2}=\sigma_{\tau}^{2(t-1)}, \sigma^{2}=\sigma^{2(t-1)}$,

$$
P_{r}=P_{r}^{(t-1)}, \sigma_{\triangle}^{2}=\sigma_{\triangle}^{2(t-1)} \text { and } \delta_{i}=\delta_{i}^{(t-1)} .
$$

(b) Generate $\mu_{1}$ from $\left[\mu_{1} \mid Y, \sigma^{2}, \sigma_{\tau}^{2},\left\{\delta_{i}\right\}\right]$ by Equation (4.11);

Generate $\sigma^{2}$ from $\left[\sigma^{2} \mid Y, \mu_{1}, \sigma_{\tau}^{2},\left\{\delta_{i}\right\}\right]$ by Equation (4.12);

Generate $\sigma_{\tau}^{2}$ from $\left[\tau^{2} \mid Y, \mu_{1}, \sigma^{2},\left\{\delta_{i}\right\}\right]$ by Equation (4.13);

Generate $P_{r}$ from $\left[P_{r} \mid \delta_{i}, \sigma_{\Delta}^{2}\right]$ by Equation (4.15);

Generate $\sigma_{\Delta}^{2}$ from $\left[\sigma_{\Delta}^{2} \mid \delta_{i}, P_{r}\right]$ by Equation (4.16);

In order to generate Compute $\delta_{i}$, first compute $a_{i}^{(t)}$ and $g_{i}^{(t)}$ by

$$
\begin{aligned}
& a_{i}^{(t)}=\frac{m}{\sigma^{2}}\left((k-i)-\frac{\sigma_{\tau}^{2}(k-i)^{2}}{k \sigma_{\tau}^{2}+\sigma^{2}}\right)+\frac{\Delta_{i}}{\sigma_{\Delta}^{2}}, \text { and } \\
& g_{i}^{(t)}=\frac{1}{\sigma^{2}}\left(1-\frac{\sigma_{\tau}^{2}(k-i)}{k \sigma_{\tau}^{2}+\sigma^{2}}\right) s_{i},
\end{aligned}
$$

where

$$
s_{i}=\sum_{j=1}^{m} \sum_{p=i+1}^{k}\left(y_{p j}-\mu_{1}-\sum_{q=1}^{p-1} \delta_{q}+\delta_{i}\right) .
$$


Second, compute the conditional posterior probability of $\delta_{i}^{(t)}=0$, (i.e. $\lambda_{i}^{(t)}$ ) for $1 \leq i \leq k-1$ by

$$
\begin{aligned}
\lambda_{i}^{(t)} & =\operatorname{Pr}\left(\delta_{i}^{(t)}=0 \mid \cdot\right) \\
& =\frac{\left(1-P_{r}\right) h\left(0 ; a_{i}^{(t)}, g_{i}^{(t)}\right)}{\left(1-P_{r}\right) h\left(0 ; a_{i}^{(t)}, g_{i}^{(t)}\right)+P_{r} \frac{1}{\sigma_{\Delta}} \int_{0}^{\infty} h\left(\delta_{i}^{(t-1)} ; a_{i}^{(t)}, g_{i}^{(t)}, \sigma_{\triangle}^{2}\right) d \delta_{i}^{(t-1)}},
\end{aligned}
$$

where

$$
h\left(\delta_{i}\right)=\frac{1}{\sqrt{2 \pi\left(\frac{1}{a_{i}}\right)}} \exp \left\{-\frac{1}{2\left(\frac{1}{a_{i}}\right)}\left(\delta_{i}-\frac{g_{i}}{a_{i}}\right)^{2}\right\} \exp \left\{\frac{g_{i}^{2}}{2 a_{i}}\right\} .
$$

Third, sample $\mathrm{Br}_{i}^{(t)}$ from a Bernoulli distribution with the success probability $\lambda_{i}^{(t)}$ by $\operatorname{Br}_{i}^{(t)} \sim \operatorname{Bern}\left(\lambda_{i}^{(t)}\right)$ :

If $\operatorname{Br}_{i}^{(t)}=0$, then $\delta_{i}^{(t)}$ sampled from $\delta_{i}^{(t)} \sim \operatorname{Normal}\left(\frac{g_{i}^{(t)}}{a_{i}^{(t)}}, \sqrt{\frac{1}{a_{i}^{(t)}}}\right) ;$

If $\mathrm{Br}_{i}^{(t)}=1$, then $\delta_{i}^{(t)}=0$.

(c) Update $\mu_{1}^{(t)}=\mu_{1}, \sigma_{\tau}^{2(t)}=\sigma_{\tau}^{2}, \sigma^{2(t)}=\sigma^{2}, P_{r}^{(t)}=P_{r}, \sigma_{\triangle}^{2(t)}=\sigma_{\triangle}^{2}$ and $\delta_{i}^{(t)}=\delta_{i}$.

Step 3: Increment $t$, then repeat Step 2. 


\section{CHAPTER 5}

\section{PRESENTATION OF SIMULATIONS}

\section{AND APPLICATIONS}

\subsection{Introduction}

In this chapter, we apply the proposed hierarchical model in Equation (4.1) into the simulations using the Gibbs sampler algorithm presented in Section 4.6. In Section 5.2, first part we propose the hypotheses for pairwise multiple comparison hypotheses for each pair of population means, the global testing hypotheses and the joint testing hypotheses. In the second part of Section 5.2, we present a detailed description for the setting of our simulation study, including setting iteration and burn-in, the true mean values, and the chosen values for the priors. In Section 5.3, we analyze the testing results for four different simulated data sets. Simulation 1 is developed to find the optimal choice of priors for

hyper-parameter $\sigma_{\triangle}^{2}$. Since the goal is to keep $\sigma_{\triangle}^{2}$ as non-informative as possible, we try several different combinations of priors $a_{0}$ and $b_{0}$ in order to get a large variance of $\sigma_{\triangle}^{2}$ and also to keep parameters estimations close to their true values and to have 
the optimal results for the proposed procedure. Simulation 2 is developed to find the optimal choice of hyper-parameter of prior $\mu_{1}$. The idea is same as simulation 1, to find an optimal combination of $\mu_{0}$ and $\tau_{0}^{2}$ values so that $\mu_{1}$ is as non-informative as possible and that parameters estimations are close to the true values. After finding the best combination for these priors, in Simulation 3, we examined the hierarchical model using parameters estimation and posterior probabilities estimation, by checking whether the estimated values are close to the true values, and we analyze whether the posterior probabilities in the hierarchical model can conduct multiple comparisons well in different data sets. Simulation 4 is to study the Type I errors of the proposed decision rules for pairwise tests, global tests and joint tests. Finally, in Section 5.4, we apply the proposed Bayesian hierarchical model to two real data sets. The first application is designed to reduce lead blood concentrations in children with elevated levels, and data is from Pediatric Research written by the TLC (Treatment of Lead-Exposed Children) Trail Group. The second application is to determine any significant difference of the midterm examination scores in a mathematical statistics course held in Fall 1998 at the University of Missouri-Columbia. Both data sets are applied to the proposed hierarchical model and directly using the Gibbs sampler algorithm with the pre-set priors in the previous sections. The results in both applications illustrate that the proposed procedure performs effectively. 


\subsection{Hypothesis Testings via Posterior Probability}

In this section, we introduce the assumptions and procedures about how to use posterior probabilities to test hypotheses about the means under the proposed hierarchical model.

Because we are interested in the hypotheses for successive pairwise comparisons of the means, $H_{0 i}: \mu_{i}=\mu_{i}+\delta_{i}$ versus $H_{1 i}: \mu_{i} \neq \mu_{i}+\delta_{i}$, or $H_{0 i}: \delta_{i}=0$ versus $H_{1 i}: \delta_{i} \neq 0$ for $i=1, \ldots, k-1$. If we assign equal prior probabilities to $H_{0 i}$, we may reject $H_{0 i}$ when $\operatorname{Pr}\left\{\delta_{i}=0 \mid Y\right\}$ is less than some predetermined cutoff value. In a traditional scheme, this cutoff value could be 0.5 . Another value is dictated by the context of the application.

For the global test of $H_{0}: \mu_{1}=\cdots=\mu_{k}$ versus $H_{1}$ : not all means are equal, we may naturally accept $H_{1}$ if at least one of the pairwise tests accepts $H_{1}, i=1, \ldots, k$; that is,

accept $H_{1}$ if $\min _{1 \leq i \leq k-1}\left\{\operatorname{Pr}\left\{\delta_{i}=0 \mid Y\right\}\right\}$ is less than the aforementioned cutoff value for rejecting $H_{0}$.

We emphasize that the preceding rules for the pairwise and global tests are formulated to be consistent. By employing these rules, the test results are coherent. Note that for the preceding rules, the initial probabilities of the individual null hypotheses, $H_{0 i}: \delta_{i}=$ $0,1 \leq i \leq k-1$, are traditionally set at $\operatorname{Pr}\left\{H_{0}\right\}=0.5$. Some might argue that this cutoff value should be even larger because the null hypothesis is often the "established theory" (Berger \& Sellke, 1987).

However, if several tests are conducted in the same experiment, assigning the initial probabilities of the individual null hypotheses to be 0.5 , i.e. $\operatorname{Pr}\left\{H_{0}\right\}=0.5$, may result in too many false rejections of the individual null hypotheses. Therefore, we may need to 
adjust the prior probability of the null hypotheses so that the Type I error rate can be effectively controlled.

Following the intuitive rule proposed by Westfall, Johnson, \& Utts (1997), in order to obtain $\operatorname{Pr}\left\{H_{0}\right\}=0.5$, for the model with a total of $k$ mutually independent priors for the $\delta_{i}$ 's , we calibrate the prior probabilities for $H_{0 i}$ via $\operatorname{Pr}\left\{H_{0 i}\right\}^{\star}=0.5^{1 / C_{2}^{k}}$.

We observe that

$$
\begin{aligned}
\operatorname{Pr}\left\{H_{0 i}\right\}=\operatorname{Pr}\left\{\delta_{i}\right. & =0\}=E\left[E\left(I_{\left\{\delta_{i}=0\right\}} \mid P_{r}, \sigma_{\triangle}^{2}\right)\right] \\
& =E\left(P_{r}\right)=\frac{a_{r}}{a_{r}+b_{r}} .
\end{aligned}
$$

Thus, if one wants the initial prior probabilities of $\operatorname{Pr}\left\{H_{0}\right\}=0.5$, then one could choose $a_{r}=b_{r}$ and select 1 as the common value. Note that for any given $k$, there exist an infinite number of choices for $a_{r}$ and $b_{r}$ that satisfy $\operatorname{Pr} H_{0 i}{ }^{\star}=0.5^{1 / C_{2}^{k}}=\frac{a_{r}}{a_{r}+b_{r}}$.

For pairwise comparisons, we adopt the conventional scheme of using 0.5 as the decision criterion for the posterior probability. For $H_{0 i}: \mu_{i}=\mu_{i}+\delta_{i}$ versus $H_{1 i}: \mu_{i} \neq \mu_{i}+\delta_{i}$, with $1 \leq i \leq k-1$, we declare $H_{1 i}$ if

$$
\operatorname{Pr}\left\{\delta_{i}=0 \mid Y\right\}<0.5
$$

where $\operatorname{Pr}\left\{\delta_{i}=0 \mid Y\right\}$ is the posterior probability of the null hypothesis resulting from the use of $\operatorname{Pr}\left\{H_{0}\right\}^{\star}$.

For the global test of $H_{0}: \mu_{1}=\cdots=\mu_{k}$ versus $H_{1}$ : Not all are equal, we declare $H_{1}$ 
if at least one of the pairwise tests declares $H_{1}$, i.e., if

$$
\min _{1 \leq i \leq k-1}\left\{\operatorname{Pr}\left\{\delta_{i}=0 \mid Y\right\}\right\}<0.5
$$

One could conduct the global test based on the joint posterior probability of the $\delta_{i}$ 's, by rejecting the global null $H_{0}$ if

$$
\operatorname{Pr}\left\{\bigcap_{i=1}^{k-1} \delta_{i}=0 \mid Y\right\}<0.5 .
$$

In this dissertation, we recommend conducting the test based on (5.2). First, the test based on (5.3) is not compatible with the tests in (5.1). That is, using the test in (5.3) and the pairwise comparisons in (5.1) may lead to inconsistent decisions. Second, we found that the global test based on (5.3) may be too liberal, i.e., it tends to reject $H_{0}$ more often than the test based on (5.2). The results from our simulation study and the results in the application suggest the procedure using hypotheses tests using (5.1) and (5.2) has reasonable power.

As previously mentioned in Section 4.6, we proposed the procedure to compute the posterior probabilities for the model by Gibbs sampling method. In order to see the convergence in a reasonable time period, the number of burn-in samples is taken to be 3,000 , and the subsequent 10,000 iterations are used to estimate the parameters. This MCMC chain generates several different results from the same specified model in (4.4), but with different hyper-parameter values. 
During the first simulation, we choose two data sets with initial population means $\mu=(10,10,10)^{\prime}$ for the equal mean case and $\mu=(10,11,12)^{\prime}$ for the unequal mean case. For each data set, we try different sets of values of priors of parameters of the proposed hierarchical model in Equation (4.4). Since the pairwise comparisons have been performed, we need to re-structure the data set during simulation in order to test the difference for all pairs, i.e., Group 1 vs Group 2, Group 2 vs Group 3, and Group 1 vs Group 3.

For parameter $P_{r} \sim \operatorname{BETA}\left(a_{r}, b_{r}\right)$, following the intuitive rule which proposed by Westfall et al. (1997), in order to make the prior probability of $\operatorname{Pr}\left\{H_{0}\right\}=0.5$ for the model with a total $k$ mutually independent priors for the $\delta_{i}, i=1,2,3$, we calibrate the prior probabilities for $H_{0 i}$ via $\operatorname{Pr}\left\{H_{0 i}\right\}=0.5^{1 / C_{2}^{k}}$. With $k=3$, the prior probability of $H_{0 i}$ must be closed to $0.5^{1 / C_{2}^{3}}=0.5^{1 / 3}=0.7937$. This is closely approximated by the choice $P_{r}=\operatorname{BETA}(230,60)$ which gives $\operatorname{Pr}\left\{H_{0 i}\right\}^{*}=E\left(P_{r}\right)=0.79$ for both cases that $\delta_{i}=0$ and $\delta_{i} \neq 0$.

For parameter $\mu_{1} \sim \mathrm{N}\left(\mu_{0}, \tau_{0}^{2}\right)$, we try to choose a vague normal prior with an arbitrary mean $\mu_{0}$ and a large variance $\tau_{0}^{2}$ in order to make $\mu_{1}$ as noninformative as possible.

For hyper-parameter $\sigma_{\Delta}^{2} \sim \operatorname{IG}\left(a_{0}, b_{0}\right)$, which it has the priors $a_{0}, b_{0}$, we will utilize several sets of prior values in order to check the effect of hyper-parameter $\sigma_{\Delta}^{2}$ on other parameters estimation and the posterior probabilities, then further to find an optimal prior for our result.

For each of the two data sets, we compute the posterior model parameter estimates and their variances, as well as the corresponding posterior probabilities. 


\subsection{Presentation of Results for Simulated Data}

\subsubsection{Simulation 1: Optimal priors for $\sigma_{\Delta}^{2}$}

This simulation is developed to find the optimal choice of priors for hyper-parameter $\sigma_{\triangle}^{2}$ in estimation of $\delta_{i}$, for checking whether different values of priors $a_{0}, b_{0}$ could make any difference on the simulated parameters values.

Since the goal is to keep $\sigma_{\triangle}^{2}$ as non-informative as possible, we try several different combinations of priors $a_{0}$ and $b_{0}$ in order to get a large variance of $\sigma_{\triangle}^{2}$ and also to keep parameters estimation close to their true values and to have the optimal results for the proposed multiple comparison procedure. For the prior of $\sigma_{\triangle}^{2}$, we have

$$
\sigma_{\Delta}^{2} \sim \operatorname{IG}\left(a_{0}, b_{0}\right) \quad \text { and } \quad \operatorname{Var}\left(\sigma_{\Delta}^{2}\right)=\frac{1}{b_{0}^{2}\left(a_{0}-1\right)^{2}\left(a_{0}-2\right)}
$$

We simulate data sets with different pairs of values of priors $a_{0}, b_{0}$ for increasing variance of $\sigma_{\Delta}^{2}$.

In this simulation, we have two different initial population means. First one is to test equal means $\mu=(10,10,10)^{\prime}$; the second one is to test unequal means $\mu=(10,11,12)^{\prime}$. For each data set, we simulate data with three different sample sizes, $m=10, m=30$, and $m=100$. We set the parameter values $\sigma_{\tau}^{2}=2, \sigma^{2}=1$, and $a_{r}=230, b_{r}=60$ for hyper-parameter $P_{r}$, and $\mu_{0}=0, \tau_{0}^{2}=100$ for $\mu_{1}$. In the outputs, $\mu_{1}$ corresponds to the estimated the first population mean; $\delta_{1}, \delta_{2}$ and $\delta_{3}$ correspond to the estimate difference 
between $\mu_{1}$ and $\mu_{2}, \mu_{2}$ and $\mu_{3}$, and $\mu_{1}$ and $\mu_{3}$, respectively. $\operatorname{Pr}\left(\delta_{i}=0 \mid Y\right)$ corresponds to the estimated posterior probabilities for the different $\delta_{i}$, where $i=1,2,3$.

Base on the results from Table 5.1 to Table 5.12, the estimated means for $\delta_{1}, \delta_{2}, \delta_{3}$, $\mu_{1}, \sigma^{2}$, and $\sigma_{\tau}^{2}$ are getting closer to the true parameters values as the value of $\operatorname{Var}\left(\sigma_{\Delta}^{2}\right)$ increases. Also we can see that the parameters values for the prior of $\sigma_{\Delta}^{2}$ could significantly affect the results of estimation and multiple comparisons. Therefore we choose the optimal prior $a_{o}=2.1, \quad b_{o}=0.005$ for $\sigma_{\Delta}^{2}$. At the same time, the results indicate that the parameter estimates are closer to their true values when the sample size increases, thus we have the best simulation result when sample size $m=100$. We can also observe that for each parameter estimate, when we have larger sample size, the variance becomes smaller than the one with smaller sample size. 
Table 5.1: Simulation Results for Optimal Priors of $\sigma_{\Delta}^{2}$ under Equal Population Means and Sample Size $m=10$ Part(a)

\begin{tabular}{|c|c|c|c|c|c|c|}
\hline \multicolumn{7}{|c|}{$\mu=(10,10,10)^{\prime}, \sigma_{\tau}^{2}=2, \sigma^{2}=1, \operatorname{Pr}: a_{r}=230, b_{r}=60 ; \mu_{1}: \mu_{0}=0, \tau_{0}^{2}=100$} \\
\hline \multirow[b]{3}{*}{ Parameter } & \multirow{2}{*}{\multicolumn{2}{|c|}{$\begin{array}{l}a_{o}=5, b_{o}=0.05 \\
\operatorname{Var}\left(\sigma_{\Delta}^{2}\right)=8.333\end{array}$}} & \multirow{2}{*}{\multicolumn{2}{|c|}{$\begin{array}{l}a_{o}=4, b_{o}=0.05 \\
\operatorname{Var}\left(\sigma_{\Delta}^{2}\right)=22.22\end{array}$}} & \multirow{2}{*}{\multicolumn{2}{|c|}{$\begin{array}{l}a_{o}=2.5, b_{o}=0.05 \\
\operatorname{Var}\left(\sigma_{\Delta}^{2}\right)=355.56\end{array}$}} \\
\hline & & & & & & \\
\hline & Mean & Variance & Mean & Variance & Mean & Variance \\
\hline$\mu_{1}$ & 9.389 & 0.656 & 9.402 & 0.617 & 9.367 & 0.638 \\
\hline$\delta_{1}$ & 0.090 & 0.558 & 0.148 & 0.565 & 0.150 & 0.588 \\
\hline$\delta_{2}$ & 0.027 & 0.428 & 0.026 & 0.440 & 0.009 & 0.424 \\
\hline$\delta_{3}$ & 0.064 & 0.463 & 0.075 & 0.413 & 0.081 & 0.394 \\
\hline$\sigma^{2}$ & 1.333 & 0.726 & 1.311 & 0.668 & 1.322 & 0.725 \\
\hline$\sigma_{\tau}^{2}$ & 2.322 & 2.781 & 2.322 & 3.249 & 2.307 & 2.803 \\
\hline \multicolumn{7}{|c|}{ Posterior Probability } \\
\hline $\operatorname{Pr}\left(\delta_{1}=0 \mid Y\right)$ & 0.215 & & 0.215 & & 0.278 & \\
\hline $\operatorname{Pr}\left(\delta_{2}=0 \mid Y\right)$ & 0.225 & & 0.246 & & 0.318 & \\
\hline $\operatorname{Pr}\left(\delta_{3}=0 \mid Y\right)$ & 0.223 & & 0.261 & & 0.325 & \\
\hline
\end{tabular}


Table 5.2: Simulation Results for Optimal Priors of $\sigma_{\Delta}^{2}$ under Equal Population Means and Sample Size $m=10$ Part(b)

\begin{tabular}{|c|c|c|c|c|c|c|}
\hline \multicolumn{7}{|c|}{$\mu=(10,10,10)^{\prime}, \sigma_{\tau}^{2}=2, \sigma^{2}=1, \operatorname{Pr}: a_{r}=230, b_{r}=60 ; \mu_{1}: \mu_{0}=0, \tau_{0}^{2}=100$} \\
\hline \multirow[b]{3}{*}{ Parameter } & \multirow{2}{*}{\multicolumn{2}{|c|}{$\begin{array}{l}a_{o}=2.1, b_{o}=0.05 \\
\operatorname{Var}\left(\sigma_{\Delta}^{2}\right)=3305.79\end{array}$}} & \multirow{2}{*}{\multicolumn{2}{|c|}{$\begin{array}{c}a_{o}=2.1, b_{o}=0.02 \\
\operatorname{Var}\left(\sigma_{\Delta}^{2}\right)=20661\end{array}$}} & \multirow{2}{*}{\multicolumn{2}{|c|}{$\begin{array}{c}a_{o}=2.1, b_{o}=0.005 \\
\operatorname{Var}\left(\sigma_{\Delta}^{2}\right)=330578\end{array}$}} \\
\hline & & & & & & \\
\hline & Mean & Variance & Mean & Variance & Mean & Variance \\
\hline$\mu_{1}$ & 9.395 & 0.627 & 9.407 & 0.589 & 9.434 & 0.518 \\
\hline$\delta_{1}$ & 0.124 & 0.533 & 0.105 & 0.532 & 0.086 & 0.384 \\
\hline$\delta_{2}$ & 0.028 & 0.397 & 0.022 & 0.375 & 0.027 & 0.252 \\
\hline$\delta_{3}$ & 0.063 & 0.398 & 0.050 & 0.374 & 0.039 & 0.263 \\
\hline$\sigma^{2}$ & 1.322 & 0.794 & 1.277 & 0.844 & 1.180 & 0.575 \\
\hline$\sigma_{\tau}^{2}$ & 2.365 & 2.996 & 2.317 & 2.927 & 2.338 & 2.657 \\
\hline \multicolumn{7}{|c|}{ Posterior Probability } \\
\hline $\operatorname{Pr}\left(\delta_{1}=0 \mid Y\right)$ & 0.304 & & 0.419 & & 0.6122 & \\
\hline $\operatorname{Pr}\left(\delta_{2}=0 \mid Y\right)$ & 0.352 & & 0.466 & & 0.6745 & \\
\hline $\operatorname{Pr}\left(\delta_{3}=0 \mid Y\right)$ & 0.344 & & 0.465 & & 0.6760 & \\
\hline
\end{tabular}


Table 5.3: Simulation Results for Optimal Priors of $\sigma_{\Delta}^{2}$ under Equal Population Means and Sample Size $m=30$ Part(a)

\begin{tabular}{|c|c|c|c|c|c|c|}
\hline \multicolumn{7}{|c|}{$\mu=(10,10,10)^{\prime}, \sigma_{\tau}^{2}=2, \sigma^{2}=1, \operatorname{Pr}: a_{r}=230, b_{r}=60 ; \mu_{1}: \mu_{0}=0, \tau_{0}^{2}=100$} \\
\hline \multirow[b]{3}{*}{ Parameter } & \multirow{2}{*}{\multicolumn{2}{|c|}{$\begin{array}{l}a_{o}=5, b_{o}=0.05 \\
\operatorname{Var}\left(\sigma_{\Delta}^{2}\right)=8.333\end{array}$}} & \multirow{2}{*}{\multicolumn{2}{|c|}{$\begin{array}{l}a_{o}=4, b_{o}=0.05 \\
\operatorname{Var}\left(\sigma_{\Delta}^{2}\right)=22.22\end{array}$}} & \multirow{2}{*}{\multicolumn{2}{|c|}{$\begin{array}{c}a_{o}=2.5, b_{o}=0.05 \\
\operatorname{Var}\left(\sigma_{\Delta}^{2}\right)=355.56\end{array}$}} \\
\hline & & & & & & \\
\hline & Mean & Variance & Mean & Variance & Mean & Variance \\
\hline$\mu_{1}$ & 9.591 & 0.180 & 9.609 & 0.179 & 9.595 & 0.165 \\
\hline$\delta_{1}$ & 0.002 & 0.167 & 0.014 & 0.166 & 0.014 & 0.148 \\
\hline$\delta_{2}$ & 0.159 & 0.129 & 0.145 & 0.135 & 0.140 & 0.127 \\
\hline$\delta_{3}$ & 0.128 & 0.129 & 0.124 & 0.135 & 0.120 & 0.120 \\
\hline$\sigma^{2}$ & 1.318 & 0.093 & 1.314 & 0.091 & 1.301 & 0.087 \\
\hline$\sigma_{\tau}^{2}$ & 1.949 & 0.460 & 1.970 & 0.496 & 1.957 & 0.434 \\
\hline \multicolumn{7}{|c|}{ Posterior Probability } \\
\hline $\operatorname{Pr}\left(\delta_{1}=0 \mid Y\right)$ & 0.304 & & 0.336 & & 0.402 & \\
\hline $\operatorname{Pr}\left(\delta_{2}=0 \mid Y\right)$ & 0.307 & & 0.336 & & 0.405 & \\
\hline $\operatorname{Pr}\left(\delta_{3}=0 \mid Y\right)$ & 0.305 & & 0.345 & & 0.414 & \\
\hline
\end{tabular}


Table 5.4: Simulation Results for Optimal Priors of $\sigma_{\Delta}^{2}$ under Equal Population Means and Sample Size $m=30$ Part(b)

\begin{tabular}{|c|c|c|c|c|c|c|}
\hline \multicolumn{7}{|c|}{$\mu=(10,10,10)^{\prime}, \sigma_{\tau}^{2}=2, \sigma^{2}=1, \operatorname{Pr}: a_{r}=230, b_{r}=60 ; \mu_{1}: \mu_{0}=0, \tau_{0}^{2}=100$} \\
\hline \multirow[b]{3}{*}{ Parameter } & \multirow{2}{*}{\multicolumn{2}{|c|}{$\begin{array}{l}a_{o}=2.1, b_{o}=0.05 \\
\operatorname{Var}\left(\sigma_{\Delta}^{2}\right)=3305.79\end{array}$}} & \multirow{2}{*}{\multicolumn{2}{|c|}{$\begin{array}{c}a_{o}=2.1, b_{o}=0.02 \\
\operatorname{Var}\left(\sigma_{\Delta}^{2}\right)=20661\end{array}$}} & \multirow{2}{*}{\multicolumn{2}{|c|}{$\begin{array}{c}a_{o}=2.1, b_{o}=0.005 \\
\operatorname{Var}\left(\sigma_{\Delta}^{2}\right)=330578\end{array}$}} \\
\hline & & & & & & \\
\hline & Mean & Variance & Mean & Variance & Mean & Variance \\
\hline$\mu_{1}$ & 9.598 & 0.175 & 9.597 & 0.157 & 9.632 & 0.134 \\
\hline$\delta_{1}$ & 0.012 & 0.151 & 0.020 & 0.129 & 0.016 & 0.076 \\
\hline$\delta_{2}$ & 0.138 & 0.122 & 0.116 & 0.105 & 0.074 & 0.067 \\
\hline$\delta_{3}$ & 0.120 & 0.120 & 0.098 & 0.101 & 0.064 & 0.065 \\
\hline$\sigma^{2}$ & 1.304 & 0.088 & 1.294 & 0.092 & 1.261 & 0.075 \\
\hline$\sigma_{\tau}^{2}$ & 1.965 & 0.467 & 1.962 & 0.455 & 1.968 & 0.457 \\
\hline \multicolumn{7}{|c|}{ Posterior Probability } \\
\hline $\operatorname{Pr}\left(\delta_{1}=0 \mid Y\right)$ & 0.437 & & 0.563 & & 0.762 & \\
\hline $\operatorname{Pr}\left(\delta_{2}=0 \mid Y\right)$ & 0.438 & & 0.582 & & 0.765 & \\
\hline $\operatorname{Pr}\left(\delta_{3}=0 \mid Y\right)$ & 0.442 & & 0.582 & & 0.771 & \\
\hline
\end{tabular}


Table 5.5: Simulation Results for Optimal Priors of $\sigma_{\Delta}^{2}$ under Equal Population Means and Sample Size $m=100$ Part(a)

\begin{tabular}{|c|c|c|c|c|c|c|}
\hline \multicolumn{7}{|c|}{$\mu=(10,10,10)^{\prime}, \sigma_{\tau}^{2}=2, \sigma^{2}=1, \operatorname{Pr}: a_{r}=230, b_{r}=60 ; \mu_{1}: \mu_{0}=0, \tau_{0}^{2}=100$} \\
\hline \multirow[b]{3}{*}{ Parameter } & \multirow{2}{*}{\multicolumn{2}{|c|}{$\begin{array}{l}a_{o}=5, b_{o}=0.05 \\
\operatorname{Var}\left(\sigma_{\Delta}^{2}\right)=8.333\end{array}$}} & \multirow{2}{*}{\multicolumn{2}{|c|}{$\begin{array}{l}a_{o}=4, b_{o}=0.05 \\
\operatorname{Var}\left(\sigma_{\Delta}^{2}\right)=22.22\end{array}$}} & \multirow{2}{*}{\multicolumn{2}{|c|}{$\begin{array}{l}a_{o}=2.5, b_{o}=0.05 \\
\operatorname{Var}\left(\sigma_{\Delta}^{2}\right)=355.56\end{array}$}} \\
\hline & & & & & & \\
\hline & Mean & Variance & Mean & Variance & Mean & Variance \\
\hline$\mu_{1}$ & 10.24 & 0.052 & 10.230 & 0.051 & 10.240 & 0.048 \\
\hline$\delta_{1}$ & 0.009 & 0.041 & 0.002 & 0.041 & 0.019 & 0.035 \\
\hline$\delta_{2}$ & 0.097 & 0.037 & 0.106 & 0.037 & 0.091 & 0.034 \\
\hline$\delta_{3}$ & 0.097 & 0.036 & 0.090 & 0.035 & 0.085 & 0.033 \\
\hline$\sigma^{2}$ & 1.057 & 0.013 & 1.057 & 0.013 & 1.056 & 0.013 \\
\hline$\sigma_{\tau}^{2}$ & 1.980 & 0.118 & 1.981 & 0.114 & 1.985 & 0.113 \\
\hline \multicolumn{7}{|c|}{ Posterior Probability } \\
\hline $\operatorname{Pr}\left(\delta_{1}=0 \mid Y\right)$ & 0.428 & & 0.465 & & 0.532 & \\
\hline $\operatorname{Pr}\left(\delta_{2}=0 \mid Y\right)$ & 0.405 & & 0.415 & & 0.509 & \\
\hline $\operatorname{Pr}\left(\delta_{3}=0 \mid Y\right)$ & 0.408 & & 0.435 & & 0.515 & \\
\hline
\end{tabular}


Table 5.6: Simulation Results for Optimal Priors of $\sigma_{\Delta}^{2}$ under Equal Population Means and Sample Size $m=100$ Part(b)

\begin{tabular}{|c|c|c|c|c|c|c|}
\hline \multicolumn{7}{|c|}{$\mu=(10,10,10)^{\prime}, \sigma_{\tau}^{2}=2, \sigma^{2}=1, \operatorname{Pr}: a_{r}=230, b_{r}=60 ; \mu_{1}: \mu_{0}=0, \tau_{0}^{2}=100$} \\
\hline \multirow[b]{3}{*}{ Parameter } & \multirow{2}{*}{\multicolumn{2}{|c|}{$\begin{array}{l}a_{o}=2.1, b_{o}=0.05 \\
\operatorname{Var}\left(\sigma_{\Delta}^{2}\right)=3305.79\end{array}$}} & \multirow{2}{*}{\multicolumn{2}{|c|}{$\begin{array}{c}a_{o}=2.1, b_{o}=0.02 \\
\operatorname{Var}\left(\sigma_{\Delta}^{2}\right)=20661\end{array}$}} & \multirow{2}{*}{\multicolumn{2}{|c|}{$\begin{array}{c}a_{o}=2.1, b_{o}=0.005 \\
\operatorname{Var}\left(\sigma_{\Delta}^{2}\right)=330578\end{array}$}} \\
\hline & & & & & & \\
\hline & Mean & Variance & Mean & Variance & Mean & Variance \\
\hline$\mu_{1}$ & 10.24 & 0.046 & 10.24 & 0.042 & 10.26 & 0.035 \\
\hline$\delta_{1}$ & 0.025 & 0.033 & 0.017 & 0.029 & 0.016 & 0.020 \\
\hline$\delta_{2}$ & 0.081 & 0.032 & 0.070 & 0.028 & 0.044 & 0.018 \\
\hline$\delta_{3}$ & 0.083 & 0.031 & 0.066 & 0.027 & 0.043 & 0.019 \\
\hline$\sigma^{2}$ & 1.054 & 0.013 & 1.052 & 0.013 & 1.044 & 0.012 \\
\hline$\sigma_{\tau}^{2}$ & 1.982 & 0.118 & 1.980 & 0.114 & 1.983 & 0.113 \\
\hline \multicolumn{7}{|c|}{ Posterior Probability } \\
\hline $\operatorname{Pr}\left(\delta_{1}=0 \mid Y\right)$ & 0.565 & & 0.673 & & 0.816 & \\
\hline $\operatorname{Pr}\left(\delta_{2}=0 \mid Y\right)$ & 0.546 & & 0.656 & & 0.809 & \\
\hline $\operatorname{Pr}\left(\delta_{3}=0 \mid Y\right)$ & 0.550 & & 0.656 & & 0.816 & \\
\hline
\end{tabular}


Table 5.7: Simulation Results for Optimal Priors of $\sigma_{\Delta}^{2}$ under Unequal Population Means and Sample Size $m=10$ Part(a)

\begin{tabular}{|c|c|c|c|c|c|c|}
\hline \multicolumn{7}{|c|}{$\mu=(10,11,12)^{\prime}, \sigma_{\tau}^{2}=2, \sigma^{2}=1, \operatorname{Pr}: a_{r}=230, b_{r}=60 ; \mu_{1}: \mu_{0}=0, \tau_{0}^{2}=100$} \\
\hline \multirow[b]{3}{*}{ Parameter } & \multirow{2}{*}{\multicolumn{2}{|c|}{$\begin{array}{l}a_{o}=5, b_{o}=0.05 \\
\operatorname{Var}\left(\sigma_{\Delta}^{2}\right)=8.333\end{array}$}} & \multirow{2}{*}{\multicolumn{2}{|c|}{$\begin{array}{l}a_{o}=4, b_{o}=0.05 \\
\operatorname{Var}\left(\sigma_{\Delta}^{2}\right)=22.22\end{array}$}} & \multirow{2}{*}{\multicolumn{2}{|c|}{$\begin{array}{l}a_{o}=2.5, b_{o}=0.05 \\
\operatorname{Var}\left(\sigma_{\Delta}^{2}\right)=355.56\end{array}$}} \\
\hline & & & & & & \\
\hline & Mean & Variance & Mean & Variance & Mean & Variance \\
\hline$\mu_{1}$ & 10.95 & 0.803 & 10.96 & 0.805 & 10.95 & 0.846 \\
\hline$\delta_{1}$ & 0.862 & 0.629 & 0.876 & 0.632 & 0.884 & 0.636 \\
\hline$\delta_{2}$ & 1.050 & 0.504 & 1.050 & 0.528 & 1.055 & 0.536 \\
\hline$\delta_{3}$ & 1.768 & 0.518 & 1.821 & 0.522 & 1.858 & 0.513 \\
\hline$\sigma^{2}$ & 1.131 & 0.609 & 1.122 & 0.569 & 1.134 & 0.689 \\
\hline$\sigma_{\tau}^{2}$ & 2.383 & 2.699 & 2.391 & 2.711 & 2.443 & 3.249 \\
\hline \multicolumn{7}{|c|}{ Posterior Probability } \\
\hline $\operatorname{Pr}\left(\delta_{1}=0 \mid Y\right)$ & 0.1043 & & 0.1069 & & 0.1431 & \\
\hline $\operatorname{Pr}\left(\delta_{2}=0 \mid Y\right)$ & 0.0651 & & 0.0750 & & 0.0949 & \\
\hline $\operatorname{Pr}\left(\delta_{3}=0 \mid Y\right)$ & 0.1333 & & 0.0107 & & 0.0103 & \\
\hline
\end{tabular}


Table 5.8: Simulation Results for Optimal Priors of $\sigma_{\Delta}^{2}$ under Unequal Population Means and Sample Size $m=10$ Part(b)

\begin{tabular}{|c|c|c|c|c|c|c|}
\hline \multicolumn{7}{|c|}{$\mu=(10,11,12)^{\prime}, \sigma_{\tau}^{2}=2, \sigma^{2}=1, \operatorname{Pr}: a_{r}=230, b_{r}=60 ; \mu_{1}: \mu_{0}=0, \tau_{0}^{2}=100$} \\
\hline \multirow[b]{3}{*}{ Parameter } & \multirow{2}{*}{\multicolumn{2}{|c|}{$\begin{array}{c}a_{o}=2.1, b_{o}=0.05 \\
\operatorname{Var}\left(\sigma_{\Delta}^{2}\right)=3305.79\end{array}$}} & \multirow{2}{*}{\multicolumn{2}{|c|}{$\begin{array}{c}a_{o}=2.1, b_{o}=0.02 \\
\operatorname{Var}\left(\sigma_{\Delta}^{2}\right)=20661\end{array}$}} & \multirow{2}{*}{\multicolumn{2}{|c|}{$\begin{array}{c}a_{o}=2.1, b_{o}=0.005 \\
\operatorname{Var}\left(\sigma_{\Delta}^{2}\right)=330578\end{array}$}} \\
\hline & & & & & & \\
\hline & Mean & Variance & Mean & Variance & Mean & Variance \\
\hline$\mu_{1}$ & 10.910 & 0.799 & 10.96 & 0.807 & 10.92 & 0.751 \\
\hline$\delta_{1}$ & 0.899 & 0.664 & 0.812 & 0.695 & 0.809 & 0.769 \\
\hline$\delta_{2}$ & 1.062 & 0.564 & 1.074 & 0.591 & 1.016 & 0.677 \\
\hline$\delta_{3}$ & 1.824 & 0.532 & 1.874 & 0.583 & 1.780 & 0.622 \\
\hline$\sigma^{2}$ & 1.150 & 0.665 & 1.180 & 0.755 & 1.205 & 0.640 \\
\hline$\sigma_{\tau}^{2}$ & 2.402 & 3.062 & 2.388 & 2.953 & 2.363 & 2.891 \\
\hline \multicolumn{7}{|c|}{ Posterior Probability } \\
\hline $\operatorname{Pr}\left(\delta_{1}=0 \mid Y\right)$ & 0.1474 & & 0.2387 & & 0.3380 & \\
\hline $\operatorname{Pr}\left(\delta_{2}=0 \mid Y\right)$ & 0.1000 & & 0.129 & & 0.2316 & \\
\hline $\operatorname{Pr}\left(\delta_{3}=0 \mid Y\right)$ & 0.0120 & & 0.0189 & & 0.0404 & \\
\hline
\end{tabular}


Table 5.9: Simulation Results for Optimal Priors of $\sigma_{\Delta}^{2}$ under Unequal Population Means and Sample Size $m=30$ Part(a)

\begin{tabular}{|c|c|c|c|c|c|c|}
\hline \multicolumn{7}{|c|}{$\mu=(10,11,12)^{\prime}, \sigma_{\tau}^{2}=2, \sigma^{2}=1, \operatorname{Pr}: a_{r}=230, b_{r}=60 ; \mu_{1}: \mu_{0}=0, \tau_{0}^{2}=100$} \\
\hline \multirow[b]{3}{*}{ Parameter } & \multirow{2}{*}{\multicolumn{2}{|c|}{$\begin{array}{l}a_{o}=5, b_{o}=0.05 \\
\operatorname{Var}\left(\sigma_{\Delta}^{2}\right)=8.333\end{array}$}} & \multirow{2}{*}{\multicolumn{2}{|c|}{$\begin{array}{l}a_{o}=4, b_{o}=0.05 \\
\operatorname{Var}\left(\sigma_{\Delta}^{2}\right)=22.22\end{array}$}} & \multirow{2}{*}{\multicolumn{2}{|c|}{$\begin{array}{l}a_{o}=2.5, b_{o}=0.05 \\
\operatorname{Var}\left(\sigma_{\Delta}^{2}\right)=355.56\end{array}$}} \\
\hline & & & & & & \\
\hline & Mean & Variance & Mean & Variance & Mean & Variance \\
\hline$\mu_{1}$ & 10.83 & 0.485 & 10.84 & 0.481 & 10.83 & 0.471 \\
\hline$\delta_{1}$ & 1.098 & 0.215 & 1.102 & 0.212 & 1.087 & 0.217 \\
\hline$\delta_{2}$ & 1.070 & 0.162 & 1.062 & 0.161 & 1.086 & 0.171 \\
\hline$\delta_{3}$ & 2.092 & 0.156 & 2.114 & 0.159 & 2.101 & 0.162 \\
\hline$\sigma^{2}$ & 0.930 & 0.063 & 0.931 & 0.064 & 0.929 & 0.0618 \\
\hline$\sigma_{\tau}^{2}$ & 2.162 & 0.481 & 2.164 & 0.505 & 2.161 & 0.492 \\
\hline \multicolumn{7}{|c|}{ Posterior Probability } \\
\hline $\operatorname{Pr}\left(\delta_{1}=0 \mid Y\right)$ & 0.0167 & & 0.0198 & & 0.0258 & \\
\hline $\operatorname{Pr}\left(\delta_{2}=0 \mid Y\right)$ & 0.0107 & & 0.0121 & & 0.0148 & \\
\hline $\operatorname{Pr}\left(\delta_{3}=0 \mid Y\right)$ & 0.0000 & & 0.0000 & & 0.0000 & \\
\hline
\end{tabular}


Table 5.10: Simulation Results for Optimal Priors of $\sigma_{\Delta}^{2}$ under Unequal Population Means and Sample Size $m=30$ Part(b)

\begin{tabular}{|c|c|c|c|c|c|c|}
\hline \multicolumn{7}{|c|}{$\mu=(10,11,12)^{\prime}, \sigma_{\tau}^{2}=2, \sigma^{2}=1, \operatorname{Pr}: a_{r}=230, b_{r}=60 ; \mu_{1}: \mu_{0}=0, \tau_{0}^{2}=100$} \\
\hline \multirow[b]{3}{*}{ Parameter } & \multirow{2}{*}{\multicolumn{2}{|c|}{$\begin{array}{l}a_{o}=2.1, b_{o}=0.05 \\
\operatorname{Var}\left(\sigma_{\Delta}^{2}\right)=3305.79\end{array}$}} & \multirow{2}{*}{\multicolumn{2}{|c|}{$\begin{array}{c}a_{o}=2.1, b_{o}=0.02 \\
\operatorname{Var}\left(\sigma_{\Delta}^{2}\right)=20661\end{array}$}} & \multirow{2}{*}{\multicolumn{2}{|c|}{$\begin{array}{c}a_{o}=2.1, b_{o}=0.005 \\
\operatorname{Var}\left(\sigma_{\Delta}^{2}\right)=330578\end{array}$}} \\
\hline & & & & & & \\
\hline & Mean & Variance & Mean & Variance & Mean & Variance \\
\hline$\mu_{1}$ & 10.83 & 0.471 & 10.86 & 0.508 & 10.83 & 0.465 \\
\hline$\delta_{1}$ & 1.087 & 0.217 & 1.078 & 0.249 & 1.051 & 0.276 \\
\hline$\delta_{2}$ & 1.086 & 0.171 & 1.083 & 0.176 & 1.078 & 0.204 \\
\hline$\delta_{3}$ & 2.101 & 0.162 & 2.126 & 0.167 & 2.112 & 0.182 \\
\hline$\sigma^{2}$ & 0.929 & 0.0618 & 0.945 & 0.071 & 0.957 & 0.072 \\
\hline$\sigma_{\tau}^{2}$ & 2.161 & 0.492 & 2.175 & 0.509 & 2.151 & 0.496 \\
\hline \multicolumn{7}{|c|}{ Posterior Probability } \\
\hline $\operatorname{Pr}\left(\delta_{1}=0 \mid Y\right)$ & 0.0258 & & 0.0454 & & 0.0838 & \\
\hline $\operatorname{Pr}\left(\delta_{2}=0 \mid Y\right)$ & 0.0148 & & 0.0194 & & 0.0398 & \\
\hline $\operatorname{Pr}\left(\delta_{3}=0 \mid Y\right)$ & 0.0000 & & 0.0000 & & 0.0000 & \\
\hline
\end{tabular}


Table 5.11: Simulation Results for Optimal Priors of $\sigma_{\Delta}^{2}$ under Unequal Population Means and Sample Size $m=100$ Part(a)

\begin{tabular}{|c|c|c|c|c|c|c|}
\hline \multicolumn{7}{|c|}{$\mu=(10,11,12)^{\prime}, \sigma_{\tau}^{2}=2, \sigma^{2}=1, \operatorname{Pr}: a_{r}=230, b_{r}=60 ; \mu_{1}: \mu_{0}=0, \tau_{0}^{2}=100$} \\
\hline \multirow[b]{3}{*}{ Parameter } & \multirow{2}{*}{\multicolumn{2}{|c|}{$\begin{array}{l}a_{o}=5, b_{o}=0.05 \\
\operatorname{Var}\left(\sigma_{\Delta}^{2}\right)=8.333\end{array}$}} & \multirow{2}{*}{\multicolumn{2}{|c|}{$\begin{array}{l}a_{o}=4, b_{o}=0.05 \\
\operatorname{Var}\left(\sigma_{\Delta}^{2}\right)=22.22\end{array}$}} & \multirow{2}{*}{\multicolumn{2}{|c|}{$\begin{array}{l}a_{o}=2.5, b_{o}=0.05 \\
\operatorname{Var}\left(\sigma_{\Delta}^{2}\right)=355.56\end{array}$}} \\
\hline & & & & & & \\
\hline & Mean & Variance & Mean & Variance & Mean & Variance \\
\hline$\mu_{1}$ & 10.34 & 0.260 & 10.34 & 0.261 & 10.35 & 0.270 \\
\hline$\delta_{1}$ & 0.915 & 0.064 & 0.902 & 0.064 & 0.907 & 0.065 \\
\hline$\delta_{2}$ & 1.082 & 0.048 & 1.084 & 0.048 & 1.084 & 0.049 \\
\hline$\delta_{3}$ & 1.972 & 0.048 & 1.974 & 0.049 & 1.981 & 0.049 \\
\hline$\sigma^{2}$ & 1.079 & 0.014 & 1.082 & 0.014 & 1.082 & 0.014 \\
\hline$\sigma_{\tau}^{2}$ & 1.979 & 0.124 & 1.977 & 0.117 & 1.980 & 0.117 \\
\hline \multicolumn{7}{|c|}{ Posterior Probability } \\
\hline $\operatorname{Pr}\left(\delta_{1}=0 \mid Y\right)$ & 0.0007 & & 0.0010 & & 0.0002 & \\
\hline $\operatorname{Pr}\left(\delta_{2}=0 \mid Y\right)$ & 0.0000 & & 0.0000 & & 0.0000 & \\
\hline $\operatorname{Pr}\left(\delta_{3}=0 \mid Y\right)$ & 0.0000 & & 0.0000 & & 0.0000 & \\
\hline
\end{tabular}


Table 5.12: Simulation Results for Optimal Priors of $\sigma_{\Delta}^{2}$ under Unequal Population Means and Sample Size $m=100$ Part(b)

\begin{tabular}{|c|c|c|c|c|c|c|}
\hline \multicolumn{7}{|c|}{$\mu=(10,11,12)^{\prime}, \sigma_{\tau}^{2}=2, \sigma^{2}=1, \operatorname{Pr}: a_{r}=230, b_{r}=60 ; \mu_{1}: \mu_{0}=0, \tau_{0}^{2}=100$} \\
\hline \multirow[b]{3}{*}{ Parameter } & \multirow{2}{*}{\multicolumn{2}{|c|}{$\begin{array}{l}a_{o}=2.1, b_{o}=0.05 \\
\operatorname{Var}\left(\sigma_{\Delta}^{2}\right)=3305.79\end{array}$}} & \multirow{2}{*}{\multicolumn{2}{|c|}{$\begin{array}{c}a_{o}=2.1, b_{o}=0.02 \\
\operatorname{Var}\left(\sigma_{\Delta}^{2}\right)=20661\end{array}$}} & \multirow{2}{*}{\multicolumn{2}{|c|}{$\begin{array}{c}a_{o}=2.1, b_{o}=0.005 \\
\operatorname{Var}\left(\sigma_{\Delta}^{2}\right)=330578\end{array}$}} \\
\hline & & & & & & \\
\hline & Mean & Variance & Mean & Variance & Mean & Variance \\
\hline$\mu_{1}$ & 10.34 & 0.274 & 10.35 & 0.264 & 10.34 & 0.257 \\
\hline$\delta_{1}$ & 0.916 & 0.063 & 0.910 & 0.0626 & 0.914 & 0.067 \\
\hline$\delta_{2}$ & 1.083 & 0.049 & 1.090 & 0.049 & 1.085 & 0.051 \\
\hline$\delta_{3}$ & 1.978 & 0.050 & 1.992 & 0.051 & 2.004 & 0.049 \\
\hline$\sigma^{2}$ & 1.084 & 0.015 & 1.080 & 0.015 & 1.081 & 0.014 \\
\hline$\sigma_{\tau}^{2}$ & 1.974 & 0.118 & 1.965 & 0.116 & 1.969 & 0.115 \\
\hline \multicolumn{7}{|c|}{ Posterior Probability } \\
\hline $\operatorname{Pr}\left(\delta_{1}=0 \mid Y\right)$ & 0.0008 & & 0.0023 & & 0.0031 & \\
\hline $\operatorname{Pr}\left(\delta_{2}=0 \mid Y\right)$ & 0.0000 & & 0.0000 & & 0.0000 & \\
\hline $\operatorname{Pr}\left(\delta_{3}=0 \mid Y\right)$ & 0.0000 & & 0.0000 & & 0.0000 & \\
\hline
\end{tabular}




\subsubsection{Simulation 2: Optimal Priors for $\mu_{1}$}

This simulation is developed to find the optimal choice of hyper-prior for prior $\mu_{1}$ in estimation of posterior population means, for checking whether different values of hyperpriors $\mu_{0}$ and $\tau_{0}^{2}$ could make any difference on the simulated parameters values.

Since the goal is to keep $\mu_{1}$ as non-informative as possible, we try several different combinations of hyper-priors $\mu_{0}$ and $\tau_{0}^{2}$ in order to get a large variance of $\mu_{1}$ and also to keep parameters estimation close to their true values and to have the optimal results for the proposed multiple comparison procedure. For the prior of $\mu_{1}$, we have

$$
\mu_{1} \sim \mathrm{N}\left(\mu_{0}, \tau_{0}^{2}\right) \quad \text { and } \quad \operatorname{Var}\left(\mu_{1}\right)=\tau_{0}^{2}
$$

We simulate data set with different pairs of values of hyper-priors $\mu_{0}$ and $\tau_{0}^{2}$. In this simulation, we have two different true population means. First one is to test equal means $\mu=(10,10,10)^{\prime}$; the second one is to test unequal means $\mu=(10,11,12)^{\prime}$. For each data set, we simulate data with two different sample sizes, $m=30$, and $m=100$. We set the parameter values $\sigma_{\tau}^{2}=2, \sigma^{2}=1$, and $a_{r}=230, b_{r}=60$ for hyper-parameter $P_{r}$, and $a_{0}=2.1, b_{0}=0.005$ for $\sigma_{\Delta}^{2}$. In the outputs, $\mu_{1}$ corresponds to the estimated the first population mean; $\delta_{1}, \delta_{2}$ and $\delta_{3}$ correspond to the estimate difference between $\mu_{1}$ and $\mu_{2}$, $\mu_{2}$ and $\mu_{3}$, and $\mu_{1}$ and $\mu_{3}$, respectively. $\operatorname{Pr}\left(\delta_{i}=0 \mid Y\right)$ corresponds to the estimated posterior probabilities for the differences $\delta_{i}$, where $i=1,2,3$.

Base on the results from Table 5.13 to Table 5.16, the estimated means for $\delta_{1}, \delta_{2}, \delta_{3}$, $\mu_{1}, \sigma^{2}$, and $\sigma_{\tau}^{2}$ do not have significant change between different sets of hyper-priors for $\mu_{1}$. 
We can not see that parameters values for the prior of $\sigma_{\Delta}^{2}$ could significantly affect the results of estimation and multiple comparisons. Therefore we can choose any pair of the optimal hyper-prior $\mu_{0}$ and $\tau_{0}^{2}$ for $\mu_{1}$. However, the results indicate that the parameter estimates are closer to their true values when the sample size increases, thus we have the best simulation result when sample size $m=100$. We can also observe that for each parameter estimate, when we have larger sample size, the variance becomes smaller than the one with smaller sample size.

Table 5.13: Simulation Results for Optimal Priors of $\mu_{1}$ under Equal Population Means and Sample Size $m=30$

\begin{tabular}{|c|c|c|c|c|c|c|}
\hline \multicolumn{7}{|c|}{$\mu=(10,10,10)^{\prime}, \sigma_{\tau}^{2}=2, \sigma^{2}=1, \operatorname{Pr}: a_{r}=230, b_{r}=60 ; \sigma_{\Delta}^{2}: a_{o}=2.1, b_{o}=0.005$} \\
\hline \multirow[b]{2}{*}{ Parameter } & \multicolumn{2}{|c|}{$\mu_{0}=0, \tau_{0}^{2}=100$} & \multicolumn{2}{|c|}{$\mu_{0}=10, \tau_{0}^{2}=100$} & \multicolumn{2}{|c|}{$\mu_{0}=10, \tau_{0}^{2}=10000$} \\
\hline & Mean & Variance & Mean & Variance & Mean & Variance \\
\hline$\mu_{1}$ & 9.451 & 0.791 & 9.463 & 0.507 & 9.451 & 0.791 \\
\hline$\delta_{1}$ & 0.053 & 0.396 & 0.068 & 0.378 & 0.053 & 0.396 \\
\hline$\delta_{2}$ & 0.019 & 0.263 & 0.006 & 0.253 & 0.019 & 0.263 \\
\hline$\delta_{3}$ & 0.470 & 0.263 & 0.044 & 0.260 & 0.470 & 0.263 \\
\hline$\sigma^{2}$ & 1.226 & 0.791 & 1.177 & 0.549 & 1.226 & 0.791 \\
\hline$\sigma_{\tau}^{2}$ & 2.350 & 2.900 & 2.314 & 2.498 & 2.350 & 2.900 \\
\hline \multicolumn{7}{|c|}{ Posterior Probability } \\
\hline $\operatorname{Pr}\left(\delta_{1}=0 \mid Y\right)$ & 0.6167 & & 0.6101 & & 0.6167 & \\
\hline $\operatorname{Pr}\left(\delta_{2}=0 \mid Y\right)$ & 0.6647 & & 0.6819 & & 0.6647 & \\
\hline $\operatorname{Pr}\left(\delta_{3}=0 \mid Y\right)$ & 0.6573 & & 0.6771 & & 0.6573 & \\
\hline
\end{tabular}


Table 5.14: Simulation Results for Optimal Priors of $\mu_{1}$ under Equal Population Means and Sample Size $m=100$

\begin{tabular}{|c|c|c|c|c|c|c|}
\hline \multicolumn{7}{|c|}{$\mu=(10,10,10)^{\prime}, \sigma_{\tau}^{2}=2, \sigma^{2}=1, \operatorname{Pr}: a_{r}=230, b_{r}=60 ; \sigma_{\Delta}^{2}: a_{o}=2.1, b_{o}=0.005$} \\
\hline \multirow[b]{2}{*}{ Parameter } & \multicolumn{2}{|c|}{$\mu_{0}=0, \tau_{0}^{2}=100$} & \multicolumn{2}{|c|}{$\mu_{0}=10, \tau_{0}^{2}=100$} & \multicolumn{2}{|c|}{$\mu_{0}=10, \tau_{0}^{2}=10000$} \\
\hline & Mean & Variance & Mean & Variance & Mean & Variance \\
\hline$\mu_{1}$ & 10.26 & 0.035 & 10.26 & 0.036 & 10.26 & 0.037 \\
\hline$\delta_{1}$ & 0.016 & 0.020 & 0.018 & 0.019 & 0.016 & 0.021 \\
\hline$\delta_{2}$ & 0.044 & 0.018 & 0.043 & 0.018 & 0.045 & 0.020 \\
\hline$\delta_{3}$ & 0.043 & 0.019 & 0.045 & 0.019 & 0.044 & 0.020 \\
\hline$\sigma^{2}$ & 1.044 & 0.012 & 1.045 & 0.012 & 1.048 & 0.012 \\
\hline$\sigma_{\tau}^{2}$ & 1.983 & 0.113 & 1.984 & 0.115 & 1.991 & 0.118 \\
\hline \multicolumn{7}{|c|}{ Posterior Probability } \\
\hline $\operatorname{Pr}\left(\delta_{1}=0 \mid Y\right)$ & 0.8156 & & 0.8246 & & 0.8096 & \\
\hline $\operatorname{Pr}\left(\delta_{2}=0 \mid Y\right)$ & 0.8085 & & 0.8152 & & 0.8099 & \\
\hline $\operatorname{Pr}\left(\delta_{3}=0 \mid Y\right)$ & 0.8157 & & 0.8061 & & 0.8074 & \\
\hline
\end{tabular}


Table 5.15: Simulation Results for Optimal Priors of $\mu_{1}$ under Unequal Population Means and Sample Size $m=30$

\begin{tabular}{|c|c|c|c|c|c|c|}
\hline \multicolumn{7}{|c|}{$\mu=(10,11,12)^{\prime}, \sigma_{\tau}^{2}=2, \sigma^{2}=1, \operatorname{Pr}: a_{r}=230, b_{r}=60 ; \sigma_{\Delta}^{2}: a_{o}=2.1, b_{o}=0.005$} \\
\hline \multirow[b]{2}{*}{ Parameter } & \multicolumn{2}{|c|}{$\mu_{0}=0, \tau_{0}^{2}=100$} & \multicolumn{2}{|c|}{$\mu_{0}=10, \tau_{0}^{2}=100$} & \multicolumn{2}{|c|}{$\mu_{0}=10, \tau_{0}^{2}=10000$} \\
\hline & Mean & Variance & Mean & Variance & Mean & Variance \\
\hline$\mu_{1}$ & 10.83 & 0.465 & 10.87 & 0.508 & 2.157 & 0.480 \\
\hline$\delta_{1}$ & 1.051 & 0.276 & 0.999 & 0.294 & 1.013 & 0.292 \\
\hline$\delta_{2}$ & 1.078 & 0.204 & 1.104 & 0.206 & 1.107 & 0.203 \\
\hline$\delta_{3}$ & 2.112 & 0.182 & 2.120 & 0.176 & 2.109 & 0.178 \\
\hline$\sigma^{2}$ & 0.957 & 0.072 & 0.962 & 0.075 & 10.84 & 0.468 \\
\hline$\sigma_{\tau}^{2}$ & 2.151 & 0.496 & 2.143 & 0.480 & 0.959 & 0.072 \\
\hline \multicolumn{7}{|c|}{ Posterior Probability } \\
\hline $\operatorname{Pr}\left(\delta_{1}=0 \mid Y\right)$ & 0.0838 & & 0.1090 & & 0.1011 & \\
\hline $\operatorname{Pr}\left(\delta_{2}=0 \mid Y\right)$ & 0.0398 & & 0.0341 & & 0.0363 & \\
\hline $\operatorname{Pr}\left(\delta_{3}=0 \mid Y\right)$ & 0.0001 & & 0.0000 & & 0.0000 & \\
\hline
\end{tabular}


Table 5.16: Simulation Results for Optimal Priors of $\mu_{1}$ under Unequal Population Means and Sample Size $m=100$

\begin{tabular}{|c|c|c|c|c|c|c|}
\hline \multicolumn{7}{|c|}{$\mu=(10,11,12)^{\prime}, \sigma_{\tau}^{2}=2, \sigma^{2}=1, \operatorname{Pr}: a_{r}=230, b_{r}=60 ; \sigma_{\Delta}^{2}: a_{o}=2.1, b_{o}=0.005$} \\
\hline \multirow[b]{2}{*}{ Parameter } & \multicolumn{2}{|c|}{$\mu_{0}=0, \tau_{0}^{2}=100$} & \multicolumn{2}{|c|}{$\mu_{0}=10, \tau_{0}^{2}=100$} & \multicolumn{2}{|c|}{$\mu_{0}=10, \tau_{0}^{2}=10000$} \\
\hline & Mean & Variance & Mean & Variance & Mean & Variance \\
\hline$\mu_{1}$ & 10.34 & 0.257 & 10.34 & 0.267 & 10.34 & 0.270 \\
\hline$\delta_{1}$ & 0.914 & 0.067 & 0.9218 & 0.067 & 0.905 & 0.063 \\
\hline$\delta_{2}$ & 1.085 & 0.051 & 1.083 & 0.049 & 1.088 & 0.048 \\
\hline$\delta_{3}$ & 2.004 & 0.049 & 1.99 & 0.050 & 1.992 & 0.051 \\
\hline$\sigma^{2}$ & 1.081 & 0.014 & 1.081 & 0.014 & 1.081 & 0.014 \\
\hline$\sigma_{\tau}^{2}$ & 1.969 & 0.115 & 1.974 & 0.113 & 1.971 & 0.115 \\
\hline \multicolumn{7}{|c|}{ Posterior Probability } \\
\hline $\operatorname{Pr}\left(\delta_{1}=0 \mid Y\right)$ & 0.0031 & & 0.0047 & & 0.0027 & \\
\hline $\operatorname{Pr}\left(\delta_{2}=0 \mid Y\right)$ & 0.0000 & & 0.0000 & & 0.0000 & \\
\hline $\operatorname{Pr}\left(\delta_{3}=0 \mid Y\right)$ & 0.0000 & & 0.0000 & & 0.0000 & \\
\hline
\end{tabular}




\subsubsection{Simulation 3: Parameter Estimates and Posterior Probabilities}

After deciding the prior values from previous two simulations, we apply the Gibbs sampler method to parameters estimation of means and their variances. For each set of true population means, we compare the result for $m=10, m=30$, and $m=100$, with prior $P_{r}: a_{r}=100, b_{r}=40$, and $\sigma_{\Delta}^{2}: a_{0}=2.1, b_{0}=0.005$. In the outputs, $\mu_{1}$ corresponds to the estimated first population mean; $\delta_{1}, \delta_{2}$, and $\delta_{3}$ correspond to the estimate difference between $\mu_{1}$ and $\mu_{2}, \mu_{2}$ and $\mu_{3}$, and $\mu_{1}$ and $\mu_{3}$, respectively. $\operatorname{Pr}\left(\delta_{i}=0 \mid Y\right)$ corresponds to the estimated posterior probabilities for the differences $\delta_{i}$, where $i=1,2,3$.

For $\mu=(10,10,10)^{\prime}, \sigma^{2}=1$, and $\sigma_{\tau}^{2}=2$, Table 5.17 features the parameter estimates and their variances, and the posterior probabilities of $\delta_{i}=0$ for each $i$. For $m=10$, the mean parameter estimates are $(9.434,9.520,9.473)^{\prime}$, and the variance estimates are 0.384 , 0.252 , and 0.263 , respectively. Among the variances of the parameter estimates in Table 5.11, the variance of the $\sigma_{\tau}^{2}$ estimate is the largest. Note that $\sigma_{\tau}^{2}$ reflects between subject variability; its estimate is very sensitive to changes in the data. Based on the posterior probabilities, the pairwise tests favor $H_{01}, H_{02}$, and $H_{03}$, which means that there is no significant difference between $\mu_{1}$ and $\mu_{2}, \mu_{2}$ and $\mu_{3}$, and $\mu_{1}$ and $\mu_{3}$.

For $m=30$ and $m=100$, we note that the parameter estimates for means and variances are getting closer to the true parameter values when sample size increases.

When $m=100$, the estimated population means are $(10.260,10.276,10.303)^{\prime}$, and the 
variance estimates are $0.035,0.020$, and 0.018 , respectively, which is quite close to our true population mean $(10,10,10)^{\prime}$. The posterior probabilities for $\delta_{1}=0$ is $0.8156, \delta_{2}=0$ is 0.8085 , and $\delta_{3}=0$ is 0.8157 . They are all far greater than 0.5 , thus we observe that the Bayesian tests favor $H_{0 i}, i=1,2,3$, which means that there is no significant difference between $\mu_{1}$ and $\mu_{2}, \mu_{2}$ and $\mu_{3}$, and $\mu_{1}$ and $\mu_{3}$.

Table 5.17: Estimation for Parameters and Posterior Probabilities under Equal Population Means and Small Variances

\begin{tabular}{|c|c|c|c|c|c|c|}
\hline \multicolumn{7}{|c|}{$\mu=(10,10,10)^{\prime}, \sigma^{2}=1, \sigma_{\tau}^{2}=2$} \\
\hline \multirow[b]{2}{*}{ Parameter } & \multicolumn{2}{|c|}{$m=10$} & \multicolumn{2}{|c|}{$m=30$} & \multicolumn{2}{|c|}{$m=100$} \\
\hline & Mean & Variance & Mean & Variance & Mean & Variance \\
\hline$\mu_{1}$ & 9.434 & 0.518 & 9.632 & 0.134 & 10.26 & 0.035 \\
\hline$\delta_{1}$ & 0.086 & 0.384 & 0.016 & 0.076 & 0.016 & 0.020 \\
\hline$\delta_{2}$ & 0.027 & 0.252 & 0.074 & 0.067 & 0.044 & 0.018 \\
\hline$\delta_{3}$ & 0.039 & 0.263 & 0.064 & 0.065 & 0.043 & 0.019 \\
\hline$\sigma_{\tau}^{2}$ & 2.338 & 2.657 & 1.968 & 0.457 & 1.983 & 0.012 \\
\hline$\sigma^{2}$ & 1.180 & 0.575 & 1.261 & 0.075 & 1.044 & 0.113 \\
\hline \multicolumn{7}{|c|}{ Posterior Probability } \\
\hline $\operatorname{Pr}\left(\delta_{1}=0 \mid Y\right)$ & 0.6122 & & 0.7622 & & 0.8156 & \\
\hline $\operatorname{Pr}\left(\delta_{2}=0 \mid Y\right)$ & 0.6745 & & 0.7651 & & 0.8085 & \\
\hline $\operatorname{Pr}\left(\delta_{3}=0 \mid Y\right)$ & 0.6760 & & 0.7714 & & 0.8157 & \\
\hline
\end{tabular}


For $\mu=(10,10,10)^{\prime}, \sigma^{2}=20$, and $\sigma_{\tau}^{2}=50$ and $m=10$, Table 5.18 features the mean parameter estimates are $(9.503,10.913,10.301)^{\prime}$, and the variance estimates are 12.65 , 10.65, and 11.02, respectively. The variance of the $\sigma_{\tau}^{2}$ estimate is still the largest. But the posterior probability for $\delta_{1}=0$ is $0.2537, \delta_{2}=0$ is 0.2577 , and $\delta_{3}=0$ is 0.2794 , we conclude that the pairwise tests reject $H_{01}, H_{02}$, and $H_{03}$, which means that there is significant difference between $\mu_{1}$ and $\mu_{2}, \mu_{2}$ and $\mu_{3}$, and $\mu_{1}$ and $\mu_{3}$, this result is reasonable for the small sample-size case.

For $m=30$ and $m=100$, we note that the parameter estimates for means and variances are getting closer to the true parameters' value when sample size increases.

When $m=100$, the estimated population means are $(9.836,10.007,10.082)^{\prime}$, and the variance estimates are $0.9189,0.6729$, and 0.6766 , respectively. The result is still quite close to our true population mean $(10,10,10)^{\prime}$. Even though we increases the variance between and variance among the population means, all three posterior probabilities are still greater than 0.5. Thus we observe that the Bayesian tests favor $H_{01}, H_{02}$, and $H_{03}$, which means that there is no significant difference between $\mu_{1}$ and $\mu_{2}, \mu_{2}$ and $\mu_{3}$, and $\mu_{1}$ and $\mu_{3}$, and this is supporting our original conclusion. 
Table 5.18: Estimation for Parameters and Posterior Probabilities under Equal Population Means and Large Variances

\begin{tabular}{|c|c|c|c|c|c|c|}
\hline \multicolumn{7}{|c|}{$\mu=(10,10,10)^{\prime}, \sigma^{2}=20, \sigma_{\tau}^{2}=50$} \\
\hline \multirow[b]{2}{*}{ Parameter } & \multicolumn{2}{|c|}{$m=10$} & \multicolumn{2}{|c|}{$m=30$} & \multicolumn{2}{|c|}{$m=100$} \\
\hline & Mean & Variance & Mean & Variance & Mean & Variance \\
\hline$\mu_{1}$ & 9.503 & 22.79 & 8.021 & 4.712 & 9.836 & 1.180 \\
\hline$\delta_{1}$ & 1.410 & 12.65 & -1.149 & 3.827 & 0.1719 & 0.9189 \\
\hline$\delta_{2}$ & 1.051 & 10.65 & -1.104 & 3.165 & 0.2256 & 0.6729 \\
\hline$\delta_{3}$ & 0.798 & 11.02 & -2.426 & 4.055 & 0.2461 & 0.6766 \\
\hline$\sigma_{\tau}^{2}$ & 69.46 & 2241.7 & 48.94 & 260.54 & 50.51 & 77.54 \\
\hline$\sigma^{2}$ & 27.44 & 420.14 & 24.10 & 35.50 & 23.40 & 6.980 \\
\hline \multicolumn{7}{|c|}{ Posterior Probability } \\
\hline $\operatorname{Pr}\left(\delta_{1}=0 \mid Y\right)$ & 0.2537 & & 0.3333 & & 0.5196 & \\
\hline $\operatorname{Pr}\left(\delta_{2}=0 \mid Y\right)$ & 0.2577 & & 0.3449 & & 0.5664 & \\
\hline $\operatorname{Pr}\left(\delta_{3}=0 \mid Y\right)$ & 0.2794 & & 0.1750 & & 0.5736 & \\
\hline
\end{tabular}


For $\mu=(10,11,12)^{\prime}, \sigma^{2}=1$, and $\sigma_{\tau}^{2}=2$, Table 5.19 features the mean parameter estimates for $m=10$ are $(10.92,11.73,12.70)^{\prime}$, and the variance estimates are $0.769,0.677$, and 0.622 , respectively. The variance of the $\sigma_{\tau}^{2}$ estimate is still the largest. The posterior probability for $\delta_{1}=0$ is $0.1480, \delta_{2}=0$ is 0.1716 , and $\delta_{3}=0$ is 0.0404 , we conclude that the pairwise tests reject $H_{01}, H_{02}$, and $H_{03}$, which means that there is significant difference between $\mu_{1}$ and $\mu_{2}, \mu_{2}$ and $\mu_{3}$, and $\mu_{1}$ and $\mu_{3}$.

For $m=30$ and $m=100$, we note that the parameter estimates for means and variances are getting closer to the true parameters' value when sample size increases.

When $m=100$, the estimated population means are $(10.34,11.25,12.34)^{\prime}$, and the variance estimates are 0.067, 0.051, and 0.049, respectively. This result is quite close to our true population mean $(10,11,12)^{\prime}$. The posterior probability for $\delta_{1}=0$ is 0.0031 , $\delta_{2}=0$ is 0.0000 , and $\delta_{3}=0$ is 0.0000 . Some of them even less than 0.0001 , thus we observe that the Bayesian tests reject $H_{01}, H_{02}$, and $H_{03}$, which means that there is significant difference between $\mu_{1}$ and $\mu_{2}, \mu_{2}$ and $\mu_{3}$, and $\mu_{1}$ and $\mu_{3}$, and the results support our original conclusion. 
Table 5.19: Estimation for Parameters and Posterior Probabilities under Unequal Population Means and Small Variances

\begin{tabular}{|c|c|c|c|c|c|c|}
\hline \multicolumn{7}{|c|}{$\mu=(10,11,12)^{\prime}, \sigma^{2}=1, \sigma_{\tau}^{2}=2$} \\
\hline \multirow[b]{2}{*}{ Parameter } & \multicolumn{2}{|c|}{$m=10$} & \multicolumn{2}{|c|}{$m=30$} & \multicolumn{2}{|c|}{$m=100$} \\
\hline & Mean & Variance & Mean & Variance & Mean & Variance \\
\hline$\mu_{1}$ & 10.92 & 0.751 & 10.83 & 0.465 & 10.34 & 0.257 \\
\hline$\delta_{1}$ & 0.809 & 0.769 & 1.051 & 0.276 & 0.914 & 0.067 \\
\hline$\delta_{2}$ & 1.016 & 0.677 & 1.078 & 0.204 & 1.085 & 0.051 \\
\hline$\delta_{3}$ & 1.780 & 0.622 & 2.112 & 0.182 & 2.004 & 0.049 \\
\hline$\sigma_{\tau}^{2}$ & 2.363 & 2.891 & 2.151 & 0.496 & 1.969 & 0.014 \\
\hline$\sigma^{2}$ & 1.205 & 0.640 & 0.957 & 0.072 & 1.081 & 0.115 \\
\hline \multicolumn{7}{|c|}{ Posterior Probability } \\
\hline $\operatorname{Pr}\left(\delta_{1}=0 \mid Y\right)$ & 0.1480 & & 0.0838 & & 0.0031 & \\
\hline $\operatorname{Pr}\left(\delta_{2}=0 \mid Y\right)$ & 0.1716 & & 0.0398 & & 0.0000 & \\
\hline $\operatorname{Pr}\left(\delta_{3}=0 \mid Y\right)$ & 0.0404 & & 0.0001 & & 0.0000 & \\
\hline
\end{tabular}


For $\mu=(10,11,12)^{\prime}, \sigma^{2}=20$, and $\sigma_{\tau}^{2}=50$, Table 5.20 features the similar result as in Table 5.13, the estimated parameters means and variances are getting closer to the true values when the sample size increases.

When $m=100$, the estimated population means estimates are $(9.768,10.485,11.812)^{\prime}$, and the variance estimates are 1.263, 1.280, and 1.219, respectively. All three posterior probabilities are all less than 0.5, thus we observe that the Bayesian tests reject $H_{01}, H_{02}$, and $H_{03}$, which means that there is significant difference between $\mu_{1}$ and $\mu_{2}, \mu_{2}$ and $\mu_{3}$, and $\mu_{1}$ and $\mu_{3}$, and the results support our original conclusion.

Table 5.20: Estimation for Parameters and Posterior Probabilities under Unequal Population Means and Large Variances

\begin{tabular}{|c|c|c|c|c|c|c|}
\hline \multicolumn{7}{|c|}{$\mu=(10,11,12)^{\prime}, \sigma^{2}=20, \sigma_{\tau}^{2}=50$} \\
\hline \multirow[b]{2}{*}{ Parameter } & \multicolumn{2}{|c|}{$m=10$} & \multicolumn{2}{|c|}{$m=30$} & \multicolumn{2}{|c|}{$m=100$} \\
\hline & Mean & Variance & Mean & Variance & Mean & Variance \\
\hline$\mu_{1}$ & 12.02 & 17.07 & 10.60 & 4.056 & 9.768 & 1.465 \\
\hline$\delta_{1}$ & 1.786 & 15.50 & 0.458 & 3.134 & 0.717 & 1.263 \\
\hline$\delta_{2}$ & 0.957 & 11.55 & 1.543 & 3.147 & 1.425 & 1.280 \\
\hline$\delta_{3}$ & 2.148 & 12.09 & 1.535 & 3.044 & 2.044 & 1.219 \\
\hline$\sigma_{\tau}^{2}$ & 29.83 & 565.29 & 46.60 & 221.34 & 54.70 & 80.68 \\
\hline$\sigma^{2}$ & 74.17 & 2706.3 & 20.14 & 27.50 & 20.75 & 5.843 \\
\hline \multicolumn{7}{|c|}{ Posterior Probability } \\
\hline $\operatorname{Pr}\left(\delta_{1}=0 \mid Y\right)$ & 0.4529 & & 0.4050 & & 0.3073 & \\
\hline $\operatorname{Pr}\left(\delta_{2}=0 \mid Y\right)$ & 0.2729 & & 0.2733 & & 0.2017 & \\
\hline $\operatorname{Pr}\left(\delta_{3}=0 \mid Y\right)$ & 0.2264 & & 0.2776 & & 0.0774 & \\
\hline
\end{tabular}




\subsection{Simulation 4: Familywise Error Rates and Tests Powers}

A simulation study has been performed to study the Type I errors of the proposed decision rules for pairwise, global tests, and joint test. Our results demonstrate that using the proposed hierarchical mixed model with the decision rules in Section 5.2 and Section 5.3 can generally keep the pairwise Type I error rate and experimentwise error rate (EER) quite small (below 0.005) and maintain reasonable test power. For instance, pairwise Type I error rates and EERs can be made smaller than those via the frequentist method, while test powers can be retained at an appropriately high level. As a result, we have provided that the treatment means in the mixed model and can do multiple comparison on each pair of them, we can use the proposed procedure to test hypotheses and avoid logical inconsistencies between the pairwise tests, global test and joint test.

We choose the case with $k=3$ groups, $m=30, \sigma^{2}=1$, and $\sigma_{\tau}^{2}=2$. From Section 5.3.1 and Section 5.3.2, we choose the hyper-parameter value by $P_{r}: a_{r}=100, b_{r}=$ 40; $\sigma_{\Delta}^{2}: a_{o}=2.1, b_{o}=0.005$. To compute the posterior probabilities of $H_{0}$ for a sample generated from Equation (4.4) with the specified parameters, Gibbs sampling method is employed. So in each Gibbs sampling chain, there are 10,000 MCMC samples in total and the burn-in number is 3,000. This MCMC chain is repeated for 5,000 samples generated from the same specified in Equation (4.1). At each Gibbs sampling chain, we perform a global test and multiple comparisons using the formal rules. When 5,000 repetitions are completed, we calculate the fraction of times of $H_{1}$ for pairwise tests, global test and joint 
test.

Table 5.21: Average Posterior Probability of the Null Hypothesis and Fraction of Times for Significant Differences in Simulations with $k=3, m=30$.

\begin{tabular}{|c|c|c|c|c|c|}
\hline \multicolumn{6}{|c|}{$\mu=(10.0,10.0,10.0)^{\prime}$} \\
\hline & $\mu_{1}$ vs $\mu_{2}$ & $\mu_{2}$ vs $\mu_{3}$ & $\mu_{1}$ vs $\mu_{3}$ & Global & Joint \\
\hline Av $\operatorname{Pr}\left\{H_{0} \mid \cdot\right\}$ & 0.8454 & 0.8682 & 0.8683 & 0.6438 & 0.6606 \\
\hline $\operatorname{Pr}\{$ reject $\}$ & 0.0022 & 0.0032 & 0.0048 & 0.0048 & 0.0838 \\
\hline \multicolumn{6}{|c|}{$\mu=(10.0,11.0,10.0)^{\prime}$} \\
\hline & $\mu_{1}$ vs $\mu_{2}$ & $\mu_{2}$ vs $\mu_{3}$ & $\mu_{1}$ vs $\mu_{3}$ & Global & Joint \\
\hline $\mathrm{Av} \operatorname{Pr}\left\{H_{0} \mid \cdot\right\}$ & 0.4952 & 0.4331 & 0.8157 & 0.2114 & 0.2461 \\
\hline $\operatorname{Pr}\{$ reject $\}$ & 0.4706 & 0.581 & 0.0212 & 0.6558 & 0.8982 \\
\hline
\end{tabular}

\begin{tabular}{cccccc}
\multicolumn{7}{c}{$\mu=(10.0,11.0,11.0)^{\prime}$} \\
& $\mu_{1}$ vs $\mu_{2}$ & $\mu_{2}$ vs $\mu_{3}$ & $\mu_{1}$ vs $\mu_{3}$ & Global & Joint \\
\hline $\operatorname{Av} \operatorname{Pr}\left\{H_{0} \mid \cdot\right\}$ & 0.3159 & 0.7732 & 0.4149 & 0.1412 & 0.1443 \\
\hline $\operatorname{Pr}\{$ reject $\}$ & 0.7382 & 0.0210 & 0.5460 & 0.7840 & 0.9920 \\
\hline
\end{tabular}

\begin{tabular}{cccccc}
\hline \multicolumn{7}{c}{$\mu=(10.0,11.0,12.0)^{\prime}$} \\
& $\mu_{1}$ vs $\mu_{2}$ & $\mu_{2}$ vs $\mu_{3}$ & $\mu_{1}$ vs $\mu_{3}$ & Global & Joint \\
\hline $\operatorname{Av} \operatorname{Pr}\left\{H_{0} \mid \cdot\right\}$ & 0.3367 & 0.4057 & 0.0054 & 0.0006 & 0.0004 \\
\hline $\operatorname{Pr}\{$ reject $\}$ & 0.6972 & 0.5398 & 0.9882 & 0.9999 & 0.9999 \\
\hline
\end{tabular}

Table 5.21 shows the results of simulation for $m=30$. For each of four sets of the specified models, there are two entries. First, $\operatorname{Av} \operatorname{Pr}\left\{H_{0} \mid \cdot\right\}$ represents the average posterior probability of the null hypothesis (i.e., $\delta_{i}=0$ ) over 5,000 samples. The value under "Global" within this entry is the product of the individual posterior probabilities of the null hypotheses by Equation (5.3); The value under "Joint" is the joint posterior probability of all $\delta_{i}=0$ by Equation (5.2). Secondly, $\operatorname{Pr}\{$ reject $\}$ represents the proportion 
of times that the pairwise and global null hypotheses are rejected. The first three values, those with $\mu_{i}$ vs $\mu_{j}$, for $i, j=1, \ldots, 3, i \neq j$, represent the frequentist pairwise Type I error rates and experimentwise error rate for these Bayesian procedures. The last value under "Joint" within this entry shows the experiment-wise error rate if the joint posterior probability of all $\delta_{i}=0$ being less than 0.5 were used as the criterion for rejecting the global null hypothesis.

The results also show that, when the test mean values are equal, then $\operatorname{Pr}\left\{H_{0} \mid \cdot\right\}$ is larger than 0.5 , and $\operatorname{Pr}\{$ reject $\}$ is small; but when the test mean values are not equal, then $\operatorname{Pr}\left\{H_{0} \mid \cdot\right\}$ is smaller than 0.5 , and $\operatorname{Pr}\{$ reject $\}$ is large. Secondly, Pr\{reject $\}$ under "Joint" is higher than that under "Global", indicating that using the joint posterior probability of all $\delta_{i}=0$ being less than 0.5 is easier to reject $H_{o}$ than using the minimum of all the posterior probabilities of $\delta_{i}=0$ being less than 0.5 .

Moreover, the pairwise Type I error rates are quite low in the table, and the experimentwise error rates are 0.0048. However, these Type I errors are not directly comparable to those for the frequentist tests because the frequentist Type I errors and the ones here are produced by using distinctive evidences, as discussed before. In fact, the low Type I error and experiment-wise error rates here imply that given the null hypothesis is correct, using the formal testing rules, the results based on our hierarchical model will have little chance to reject the null hypothesis. Thus, from this point of view, our approach shows quite effectively for hypothesis testing and allows to keep Type I error and experiment-wise error rates quite small. 


\subsection{Applications for the Proposed Bayesian}

\section{Hierarchical Model}

\subsubsection{Application on Lead Level Data}

In order to illustrate the utility of our model, we present an application based on data from a study designed to reduce lead blood concentrations in children with elevated levels. This study is a placebo-controlled, randomized trail described in a 2000 article from Pediatric Research written by the TLC (Treatment of Lead-Exposed Children) Trail Group. The data are provided and analyzed in Fitzmaurice et al. (2004) and it has been used in Shang J. (2008).

This study involved children between 12 and 22 months old who had blood lead levels between 20 and $44 \mu \mathrm{g} / \mathrm{dL}$. Most of the children were African-American (77\%) and lived in deterioration inner city housing. The participants were randomly assigned into two groups. At the outset of the study, all participants were provided with a month's supply of vitamin and mineral supplements, and their homes were inspected and cleaned based on a TLC regimen designed to suppress exposure to leaded dust. Participants in the "treatment" group were then provided with succimer capsules, whereas those in the "control" group were provided with a placebo. Blood lead levels were measure at baseline (week 0 ), week 1 , week 4 , and week 6 .

In our application, we consider blood lead levels collected for 50 of the children who did not receive the succimer capsules. Since the homes of these children were cleaned using an established TLC regimen, one might expect that the mean blood lead levels of 
these children would decrease over time, or at least remain the same.

In the context of Equation (4.4), $y_{i j}$ will denote the blood lead level for child $j(j=$ $1, \ldots, 50)$ at time $i(i=1, \ldots, 4)$. Thus, $\mu_{i}$ denote the mean blood lead level at time $i$. Thus, $\left(\mu_{1}, \mu_{2}, \mu_{3}, \mu_{4}\right)$ corresponds to (week 0, week 1 , week 4, week 6 ). In order to do two-sided pairwise multiple comparisons, we need to test each pair of these four different times. That means we need to compare week 0 vs week 1 , week 0 vs week 4 , week 0 vs week 6 , week 1 vs week 4 , week 1 vs week 6 and week 4 vs week 6 , total 6 pairs. Thus, we need to set the prior probability $\operatorname{Pr}\left\{H_{o}\right\}=0.5$ with $k=4$, the prior probability of each $H_{0}$ must be $0.5^{1 / C_{2}^{4}}=0.5^{1 / 6}=0.8909$. The choice $P_{r} \sim \operatorname{BETA}(400,50)$ yields $\operatorname{Pr}\left\{H_{0}\right\}^{\star}=E\left(P_{r}\right)=0.89$.

Table 5.22: Estimation Results for Parameters and Posterior Probabilities in Blood Lead Level Application

\begin{tabular}{lccc}
\hline \hline \multicolumn{3}{l}{ Sample means: $\bar{y}=(26.272,24.660,24.070,23.646)^{\prime}$} \\
\hline Parameter & Mean & Variance & $\operatorname{Pr}\left(\delta_{i}=0 \mid Y\right)$ \\
\hline$\delta_{1 \mathrm{~V} 2}$ & -1.628 & 1.212 & 0.2016 \\
$\delta_{1 \mathrm{~V} 3}$ & -2.127 & 1.021 & 0.0757 \\
$\delta_{1 \mathrm{~V} 4}$ & -2.275 & 1.083 & 0.0669 \\
$\delta_{2 \mathrm{~V} 3}$ & -0.375 & 0.667 & 0.6790 \\
$\delta_{2 \mathrm{~V} 4}$ & -0.319 & 0.601 & 0.7021 \\
$\delta_{3 \mathrm{~V} 4}$ & -0.065 & 0.440 & 0.7903 \\
\hline$\mu_{1}$ & 26.144 & 1.455 & \\
$\mu_{2}$ & 24.371 & 1.463 & \\
$\mu_{3}$ & 24.141 & 1.439 & \\
$\mu_{4}$ & 23.882 & 1.491 & \\
$\sigma_{\tau}^{2}$ & 6.376 & 2.612 & \\
$\sigma^{2}$ & 25.711 & 36.39 & \\
\hline \hline
\end{tabular}


The results of the data analysis are listed in Table 5.22. Based on the posterior estimates $\hat{\mu}_{1}, \hat{\mu}_{2}, \hat{\mu}_{3}$ and $\hat{\mu}_{4}$, the estimates for the mean blood lead levels $\left(\mu_{1}, \mu_{2}, \mu_{3}, \mu_{4}\right)^{\prime}$ are $(26.144,24.371,24.141,23.882)^{\prime}$. The estimates are reasonably close to the sample mean $\bar{y}=(26.272,24.660,24.070,23.646)^{\prime}$. The variance estimates for $\sigma_{\tau}^{2}$ and $\sigma^{2}$ resulting from our analysis are 25.711 and 6.376 , respectively.

The posterior probabilities and the $\delta_{i}$ estimates in Table 5.22 suggest that $\delta_{1 \mathrm{v} 2}<0$, $\delta_{1 \mathrm{~V} 3}<0$, and $\delta_{1 \mathrm{~V} 4}<0$, which indicate that $\mu_{1}>\mu_{2}, \mu_{1}>\mu_{3}$, and $\mu_{1}>\mu_{4}$. The results also suggest that $\delta_{2 v 3}=0, \delta_{2 v 4}=0$, and $\delta_{3 v 4}=0$, which indicate that $\mu_{2}=\mu_{3}$, $\mu_{2}=\mu_{4}$, and $\mu_{3}=\mu_{4}$. Thus, we conclude that the baseline mean is significantly higher than any other week means, yet the week 1, week 4 and week 6 means are not significantly different. From a physiological perspective, this conclusion is sensible. The most dramatic decrease in the blood lead level should occur during a period of time immediately following the cleaning of the home, reflecting the attenuation of environmental exposure to lead particulate. Over time, the level should begin to stabilize.

Figures 5.1-5.3 feature convergence plots that illustrate the posterior distributions for $\mu_{i}, \sigma_{\tau}^{2}$ and $\sigma^{2}$. These plots are constructed based on the 7,000 iterations (after 3,000 burnin) using the Gibbs sampler. The pattern of the lines shows that the estimated values converges to its true value after certain number of iterations. Figures 5.4-5.5 feature the posterior distribution plots for all $\delta_{i}$. The iterations are generated from the full conditional posterior distributions of the $\delta_{i}$ 's, and are therefore distributed in accordance with the posterior distributions of the $\delta_{i}$ 's. The highest peak in each plot indicates the existence of a preponderance of values for each $\delta_{i}$. These outputs provide some additional evidence. 


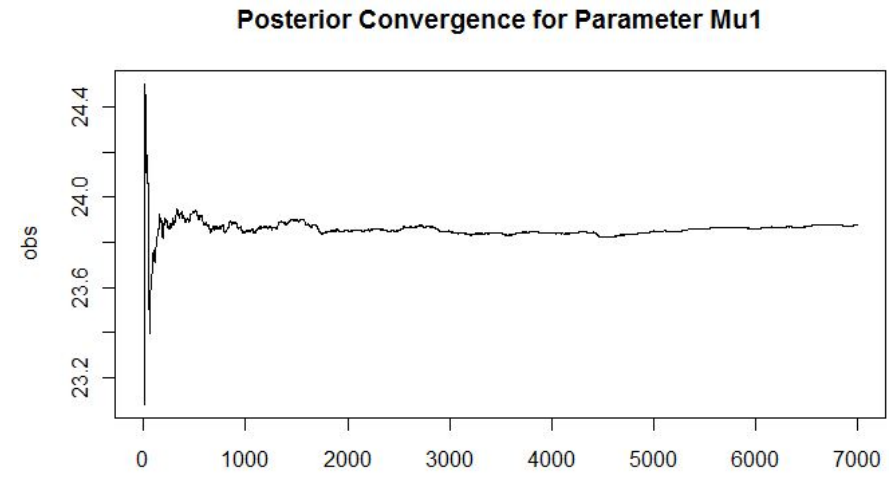

Figure 5.1: The Posterior Convergence for $\mu_{1}$ in Lead Level Data Set.

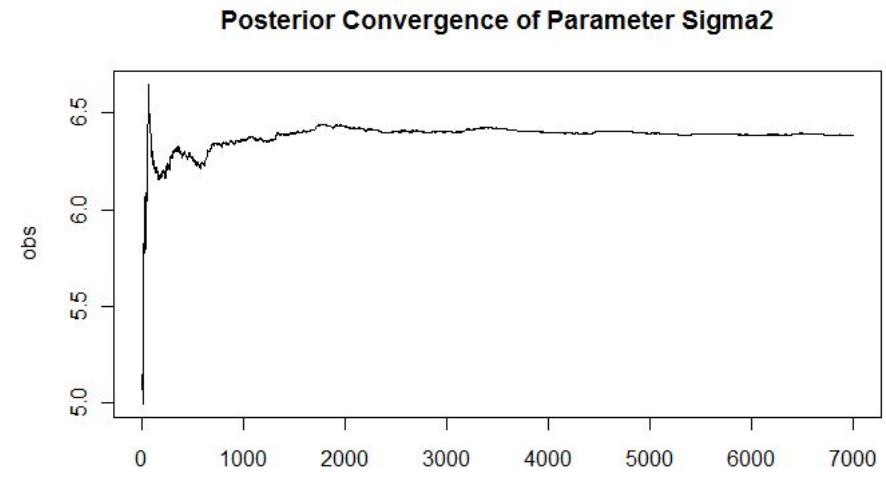

Figure 5.2: The Posterior Convergence for $\sigma^{2}$ in Lead Level Data Set.

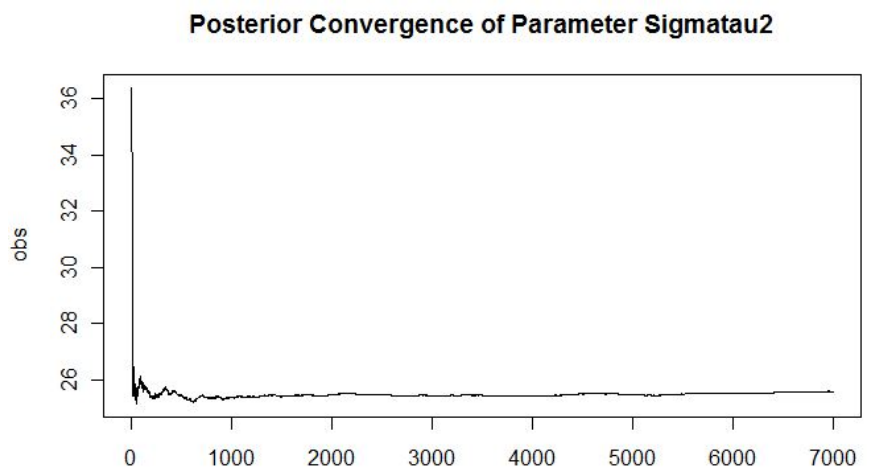

Figure 5.3: The Posterior Convergence for $\sigma_{\tau}^{2}$ in Lead Level Data Set. 


\section{Posterior Distribution for Delta 2v3}

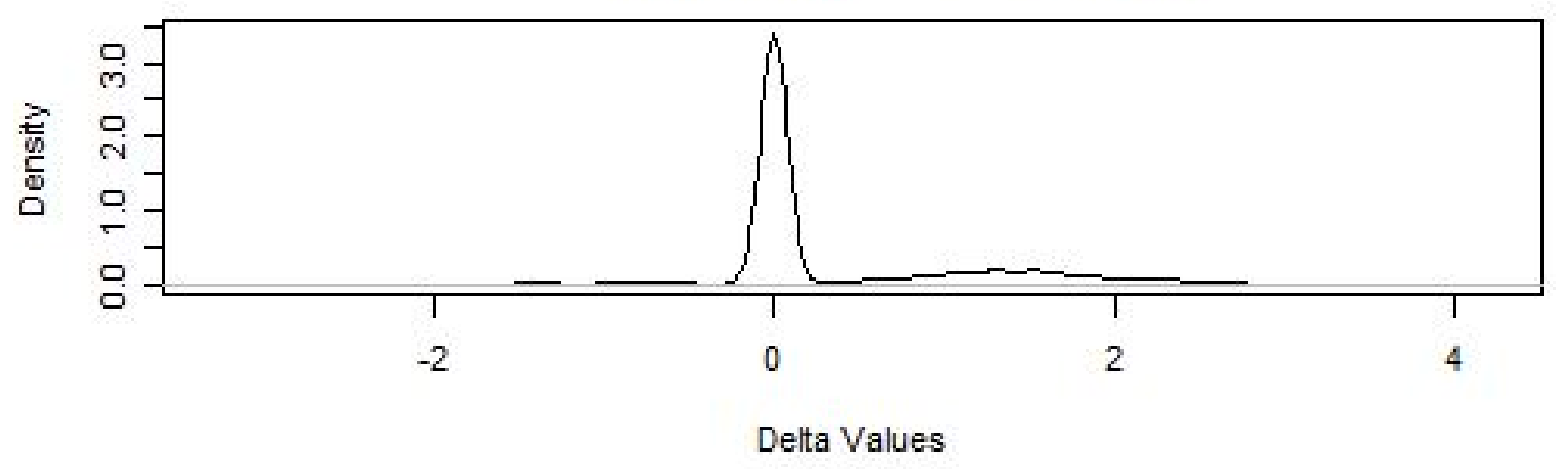

\section{Posterior Distribution for Delta 2v4}

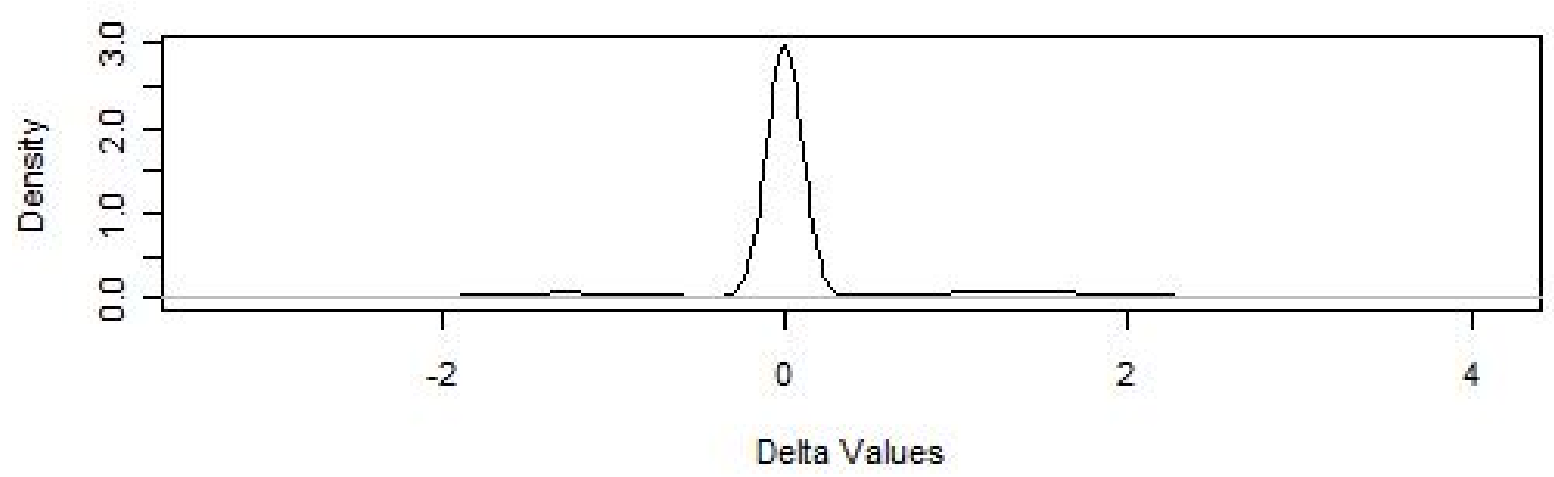

\section{Posterior Distribution for Delta 3v4}

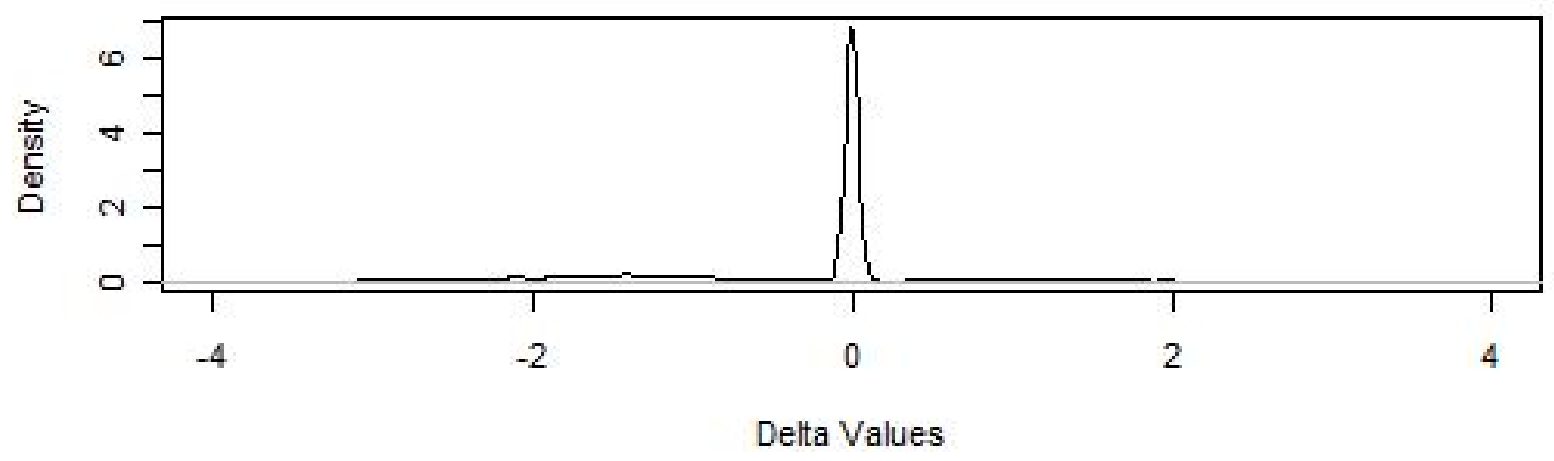

Figure 5.4: The Posterior Distributions for $\delta_{2 \mathrm{~V} 3}, \delta_{2 \mathrm{~V} 4}, \delta_{3 \mathrm{~V} 4}$ in Lead Level Data Set. 


\section{Posterior Distribution for Delta 1v2}

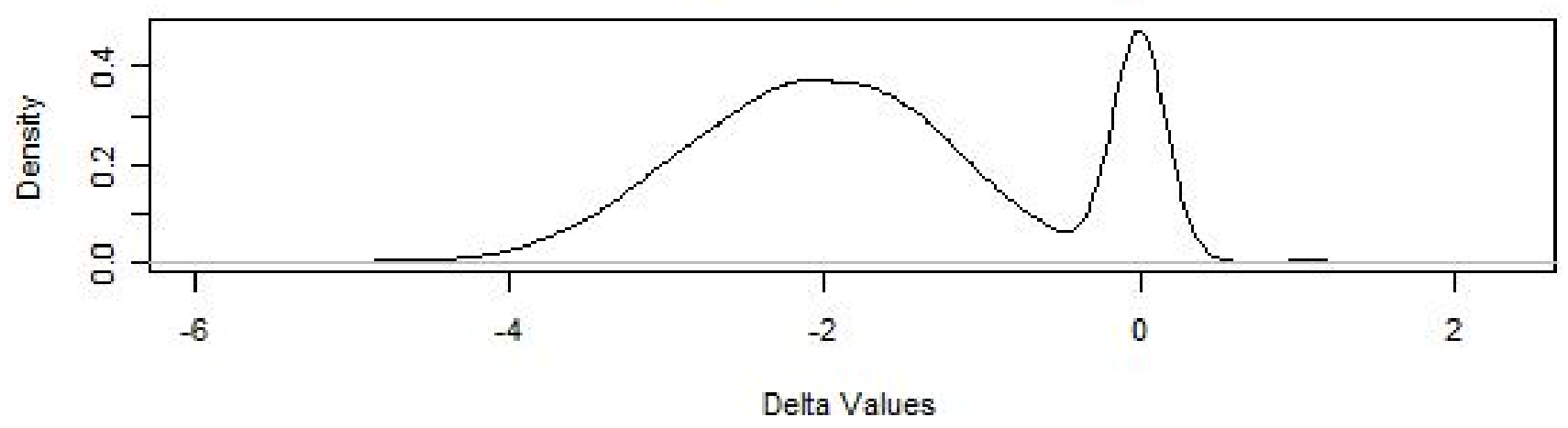

Posterior Distribution for Delta 1v3

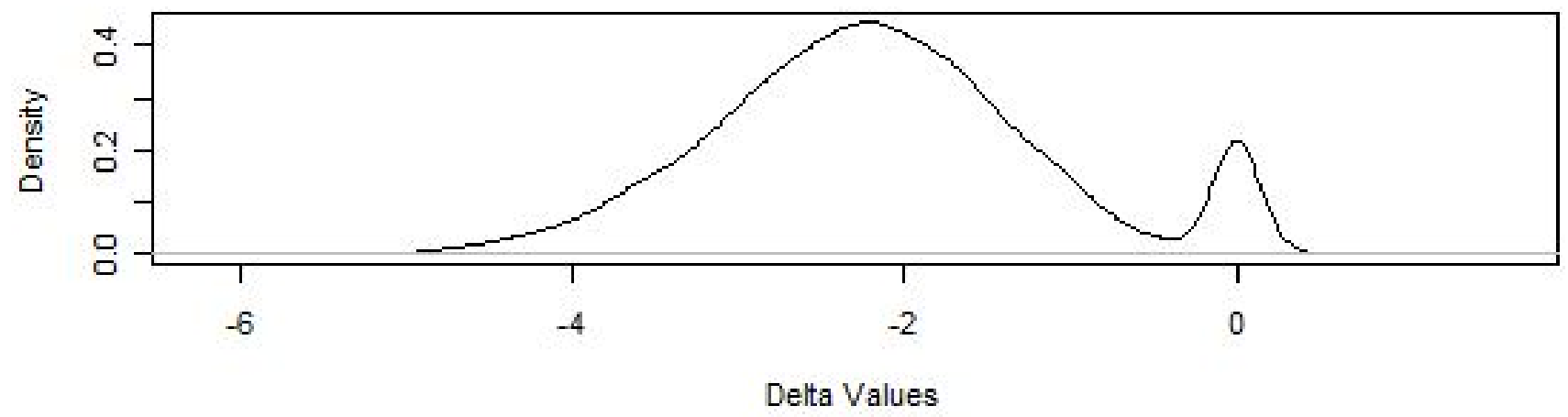

Posterior Distribution for Delta 1v4

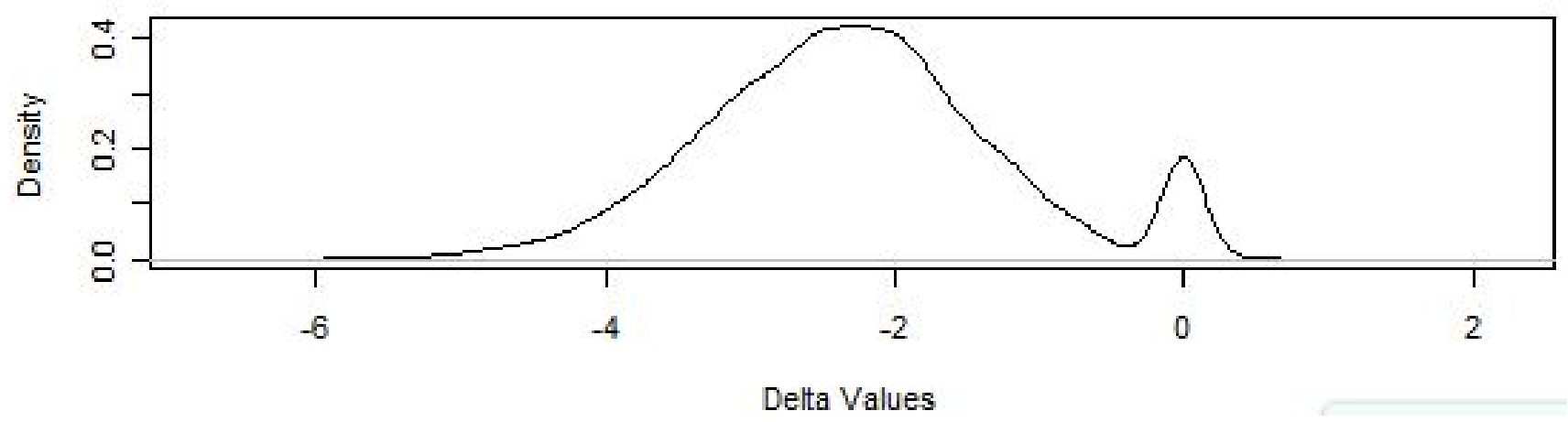

Figure 5.5: The Posterior Distributions for $\delta_{1 \mathrm{v} 2}, \delta_{1 \mathrm{v} 3}, \delta_{1 \mathrm{v} 4}$ in Lead Level Data Set. 


\subsubsection{Application on Midterm Exam Data}

To illustrate the utility of our model, we present an application based on data consisting of midterm examination scores in a mathematical statistics course held in the fall 1998 at the University of Missouri-Columbia and this data set has been used in Shang J. (2005). The data set consists of 3 exam scores for each of 72 students. For such a data set, it is reasonable to assume that the scores for a student are correlated, and yet sets of scores for different students are uncorrelated. The student effect can be regarded as random and the exam effect as fixed. We are interested is there any significant difference between mean score of these three midterm exams.

In the context of model $(4.4), y_{i j}$ will denote the midterm exam score for student $j(j=1, \ldots, 72)$ at exam $i(i=1,2,3)$. Thus, $\mu_{i}$ denote the mean midterm score at exam $i$. Thus, $\left(\mu_{1}, \mu_{2}, \mu_{3}\right)$ corresponds to (Exam 1, Exam 2, Exam 3). Thus $\delta_{1}, \delta_{2}, \delta_{3}$ corresponds the difference of Exam 1 vs Exam 2, Exam 1 vs Exam 3, and Exam 2 vs Exam 3, respectively. To set the prior probability $\operatorname{Pr}\left\{H_{o}\right\}=0.5$ with $k=3$, the prior probability of each $H_{0}$ must be $0.5^{1 / 3}=0.7937$. The choice $P_{r} \sim \operatorname{BETA}(320,60)$ yields $\operatorname{Pr}\left\{H_{0}\right\}^{\star}=E\left(P_{r}\right)=0.80$.

The results of the data analysis are listed in Table 5.23. Based on the posterior estimates $\hat{\mu}_{1}, \hat{\mu}_{2}$, and $\hat{\mu}_{3}$, the estimates for the mean midterm exam scores $\left(\mu_{1}, \mu_{2}, \mu_{3}\right)^{\prime}$ are $(71.71,80.04,74.82)^{\prime}$. The estimates are reasonably close to the sample mean $\bar{y}=$ $(69.250,84.583,73.611)^{\prime}$. The variance estimates for $\sigma_{\tau}^{2}$ and $\sigma^{2}$ resulting from our analysis are 137.80 and 130.20 , respectively. 
Table 5.23: Posterior Parameter Estimation Result for Midterm Exam Application

\begin{tabular}{lrrr}
\hline \hline \multicolumn{3}{c}{ Sample means: $\bar{y}=(69.250,84.583,73.611)^{\prime}$} \\
\hline Parameter & Mean & Variance & $\operatorname{Pr}\left(\delta_{i}=0 \mid Y\right)$ \\
\hline$\delta_{1 \mathrm{~V} 2}$ & 18.98 & 7.907 & 0.0002 \\
$\delta_{1 \mathrm{~V} 3}$ & -10.06 & 6.317 & 0.0001 \\
$\delta_{2 \mathrm{~V} 3}$ & 3.38 & 7.209 & 0.1423 \\
\hline$\mu_{1}$ & 71.71 & 51.20 & \\
$\mu_{2}$ & 80.04 & 51.88 & \\
$\mu_{3}$ & 74.82 & 51.45 & \\
$\sigma_{\tau}^{2}$ & 130.200 & 1028.600 & \\
$\sigma^{2}$ & 137.800 & 445.790 & \\
\hline
\end{tabular}

The posterior probabilities and the $\delta_{i}$ estimates in Table 5.23 suggest that $\delta_{1 \mathrm{v} 2}>0$, $\delta_{1 \mathrm{v} 3}>0$, and $\delta_{2 \mathrm{v} 3}<0$, which indicate that $\mu_{1}<\mu_{2}, \mu_{1}<\mu_{3}$, and $\mu_{2}>\mu_{3}$. Thus, we conclude that the mean midterm score in exam 2 is significantly higher than any other two midterms; the mean midterm score in exam 3 is significantly higher than in exam 1.

Figures 5.6-5.8 feature convergence plots that illustrate the posterior distribution for $\mu_{i}, \sigma_{\tau}^{2}$ and $\sigma^{2}$. These plots are constructed based on the 7,000 iterations (after 3,000 burn-in) using the Gibbs sampler. The pattern of the lines shows that the estimated values converge to its true value after certain number of iterations. Figures 5.9-5.10 feature density plot that illustrates the posterior distributions for all $\delta_{i}$. The iterations are generated from the full conditional posterior distributions of the $\delta_{i}$ 's, and are therefore distributed in accordance with the posterior distributions of the $\delta_{i}$ 's. The highest peak in each plot indicates the existence of a preponderance of values for each $\delta_{i}$. 


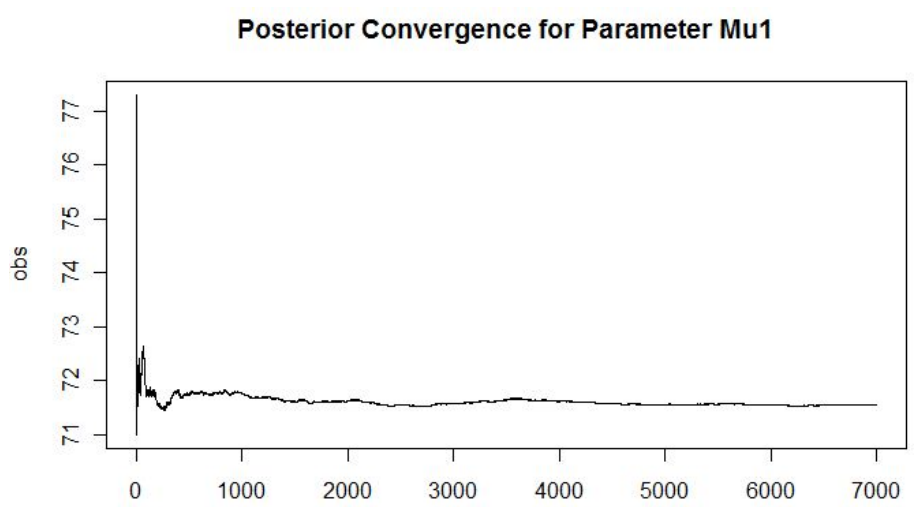

Figure 5.6: The Posterior Convergence for $\mu_{1}$ in Midterm Exam Data Set.

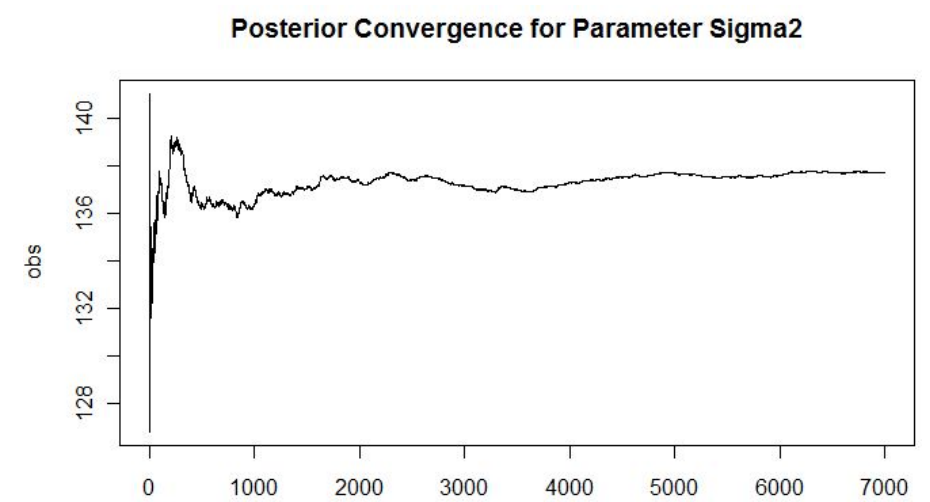

Figure 5.7: The Posterior Convergence for $\sigma^{2}$ in Midterm Exam Data Set.

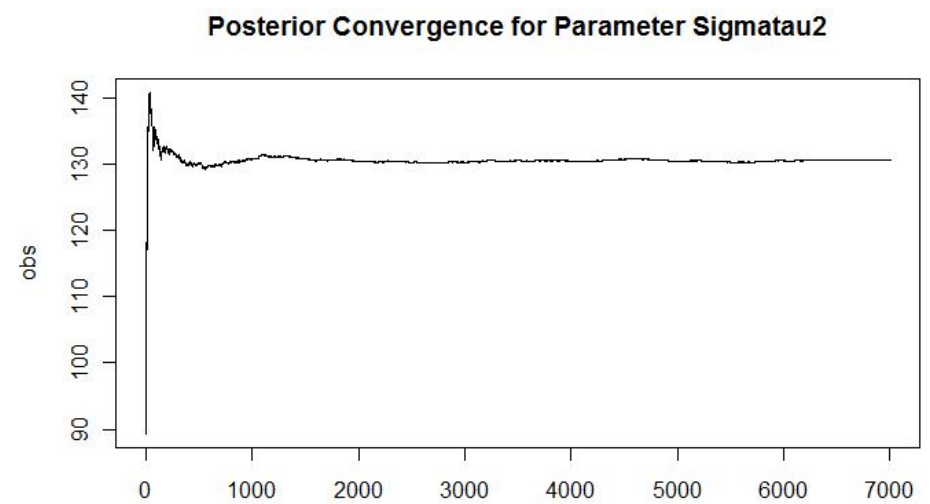

Figure 5.8: The Posterior Convergence for $\sigma_{\tau}^{2}$ in Midterm Exam Data Set. 


\section{Posterior Distribution for Delta 1v2}

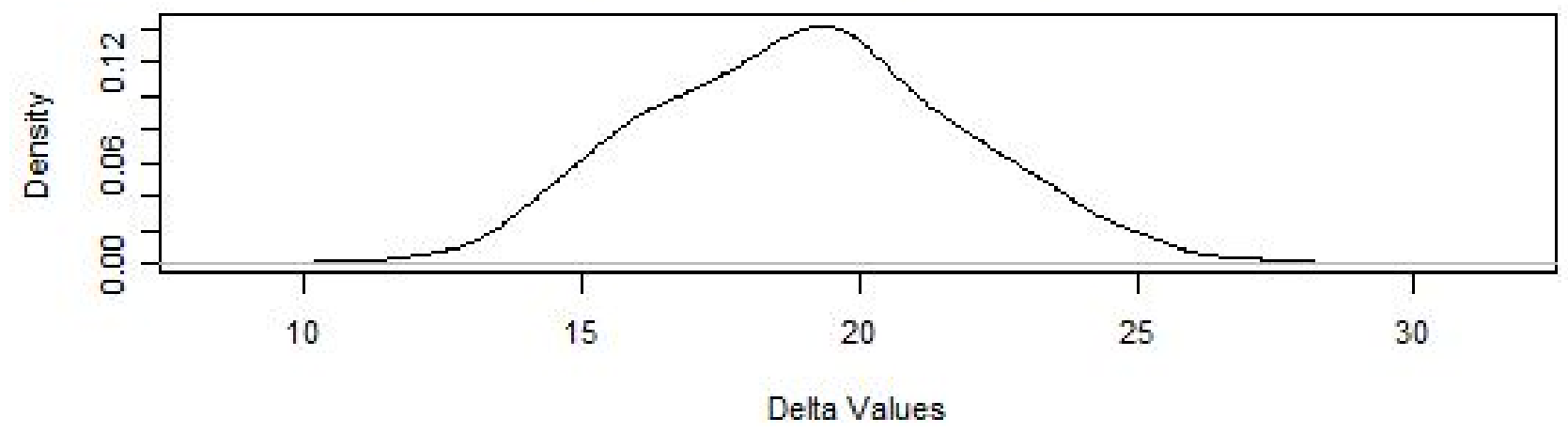

\section{Posterior Distribution for Delta 2v3}

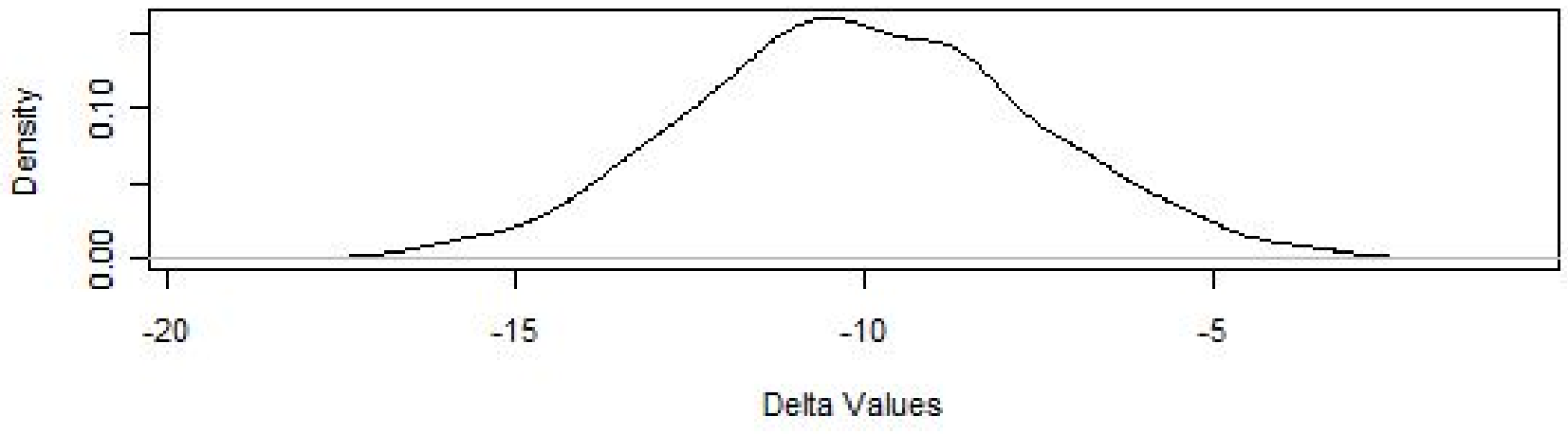

\section{Posterior Distribution for Delta 1v3}

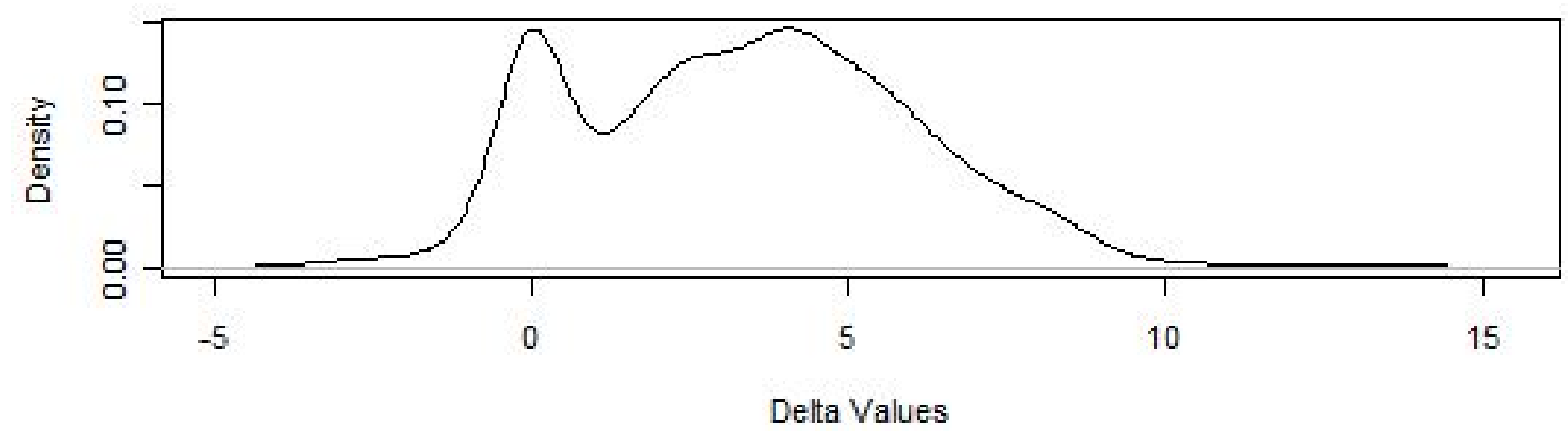

Figure 5.9: The Posterior Distributions for $\delta_{1 \mathrm{v} 2}, \delta_{1 \mathrm{v} 3}, \delta_{2 \mathrm{v} 3}$ in Midterm Exam Data Set. 


\section{CHAPTER 6}

\section{CONCLUSIONS AND FURTHER}

\section{DIRECTIONS}

In this dissertation, we are interested in multiple comparisons in a two-way ANOVA mixed model, also known as the basic repeated measures model. The result of the simulations and applications show that the proposed Bayesian hierarchical model is a $\mathrm{n}$ very effective tool for multiple comparisons under two-sided assumption. Moreover, the model is good for estimating parameters and testing the global \& joint hypotheses. Thus, with

a common decision criterion, such as one based on the posterior probability, the use of this hierarchical model can maintain logical consistency between the global test and the pairwise tests.

A further study of informative priors could also be conducted, since there are many different types of useful informative priors that could be applied in the proposed hierarchical model. For example, instead of normal distribution, there could be another way to set the mixture prior distribution for $\delta_{i}$. Meanwhile, we only demonstrate the mul- 
tiple comparison procedure for a specific type of linear mixed model: a two-way mixed model. Thus in the future study, due to different practical interests, we could devise other formulas for the Bayesian hierarchical model then derive the appropriate posterior distributions.

Following the idea of Shang J. (2011), our proposed hierarchical model can be used to deal with missing values in unbalanced data. We could combine knowledge of multiple imputation and inferential computation for multiple comparisons together, then develop an effective MCMC algorithm using either M-H sampler or Gibbs sampler. Using the same Bayesian idea we proposed in Section 2.4 and 3.6, it is feasible to provide the conditional predictive distribution for missing values and full conditional posterior distributions for parameters. 


\section{REFERENCES}

[1] Albert J. (2007), Bayesian Computation with R. Springer.

[2] Benjamini, Y. \& Yekutieli, D. (1995), Controlling the false discovery rate: a practical and powerful approach to multiple testing. Journal of the Royal Statistical Society Series B (Methodological), 57, 289-300.8

[3] Berger J. O. \$ Sellke T. (1987), Testing a Point Null Hypothesis: The Irreconcilability of P Values and Evidence. Journal of the American Statistical Association, 82, 112-122.

[4] Berry, D. (1996), Statistics: A Bayesian Perspective,, Belmont, CA: Duxbury Press.

[5] Bolstad W. M. (2010), Understanding Computational Bayesian Statistics., Wiley.

[6] Box G. E. P. \& Tiao G. C. (1992), Bayesian Inference in Statistical Analysis, WileyInterscience.

[7] Bretz, F., Hothorn, T. \& Westfall. P. (2011), Multiple Comparison Using R., CRC: Taylor \& Francis.

[8] Cavanaugh, J. E. \& Shang, J. (2005), A Diagnostic for Assessing the Influence of Cases on the Prediction of Random Effects in a Mixed model. Journal of Data Science, 3, 137-151.

[9] Choudhary, P. K. and Yin, K. (2010), Bayesian and frequentist methodologies for analyzing method comparison studies with multiple methods. Statistics in Biopharmaceutical 
Research, 2, 122-132.

[10] Clard J. S. \& Gelfand A. E. (2006), Hierarchical Modelling for The Environmental Sciences, Oxford.

[11] Congdon P. D. (2010), Applied Bayesian Hierarchical Method, CRC: Taylor \& Francis.

[12] Dunnet, C. W. (1955), A Multiple Comparison Procedure for Comparing Several Treatments with a Control. Journal of the American Statistical Association, 50, 482-491.

[13] Dunnet, C. W. (1980), Pairwise Multiple Comparisons in the Unequal Variance Case. Journal of the American Statistical Association, 87, 162-170.

[14] Fisher, R. A. (1925), Statistical Methods for Research Workers, Oliver and Boyd (Edinburgh).

[15] Fitzmaurice, G., Laird, N. \& Ware, J. (2004), Applied Longitudinal Analysis, New York: John Wiley \& Sons.

[16] Gelfand, A. E., Hills, S. E., Racine-Poon, A. \& Smith, A. F. M. (1990), Illustration of Bayesian inference in normal date problems using Gibbs sampling. Journal of the American Statistical Association, 85, 972-985.

[17] Gelfand, A. E., Smith, A. E. \& Lee, T. (1992), Bayesian analysis of constrained parameter and truncated data problems using Gibbs sampling. Journal of the American Statistical Association, 87, 523-532.

[18] Hochberg, Y. \& Tamhane, A. C. (1987), Multiple Comparison Procedure, New York: Wiley.

[19] Holm, S. (1979), A simple sequentially rejective multiple test procedure. Scandinavian Journal of Statistics, 6 (2), 6570.

[20] Hsu, J. C. (1996), Multiple Comparisons: Theory and Methods, Chapman \& Hall/CRC, 
Boca Raton, FL.

[21] Loredo, T. J. (1992), THE PROMISE OF BAYESIAN INFERENCE FOR ASTROPHYSICS. Statistical Challenges in Modern Astronomy, New York: Springer-Verlag, 275297.

[22] Molitor, J. \& Sun, D. (2002), Bayesian analysis under order functions of parameters. Environmental and Ecological Statistics 9, 179-193.

[23] Mukerjee, H. (1988), Order restricted inference in a repeated measures model. Biometrika, (75), 616-617.

[24] Nashimoto, K. \& Wright, F. T. (2008), Bayesian multiple comparisons of simply ordered means using priors with a point mass. Computational Statistics and Data Analysis, $\mathbf{5 2}, 5143-5153$.

[25] Pauler, D. K., Wakefield, J. C. \& Kass, R. E. (1990), Bayes factors and approximations for variance component models. Journal of the American Statistical Association, 94, 12421253.

[26] Rizzo M. L. (2007), Statistical computing with R., CRC: Taylor \& Francis.

[27] Robertson, T, Wright, F. T. \& Dykstra, R. L.(1988), Order Restricted Statistical Inference., New York: John Wiley \& Wiley.

[28] Shang, J., Cavanaugh, J.E. \& Wright, F. T. (2008), A Bayesian multiple comparison procedure for order-restricted mixed models. International Statistical Review, 76, 268284.

[29] Shang, J. (2011), A Bayesian Multiple Comparison Procedure for Simple OrderRestricted Mixed Models with Missing Values. Journal of Data Science, 9.

[30] Singh, B. \& Wright, F. T. (1990), Testing for and against an order restriction in mixed-effect models. Statist. Probab. Lett., 9, 195-200. 
[31] Tukey, J. W. (1953), The Problem of Multiple Comparisons, in H. I. Braun, ed., The Collected of John W. Tukey, 8, 1994, New York: Chapman \& Hall.

[32] Westfall, P. H., Johnson, W. O. \& Utts, J. M. (1997), A Bayesian look at the Bonferroni correction. Biometrika, 84, 419-427. 


\section{APPENDICES}

\section{R Program for Examples in Section 3.7}

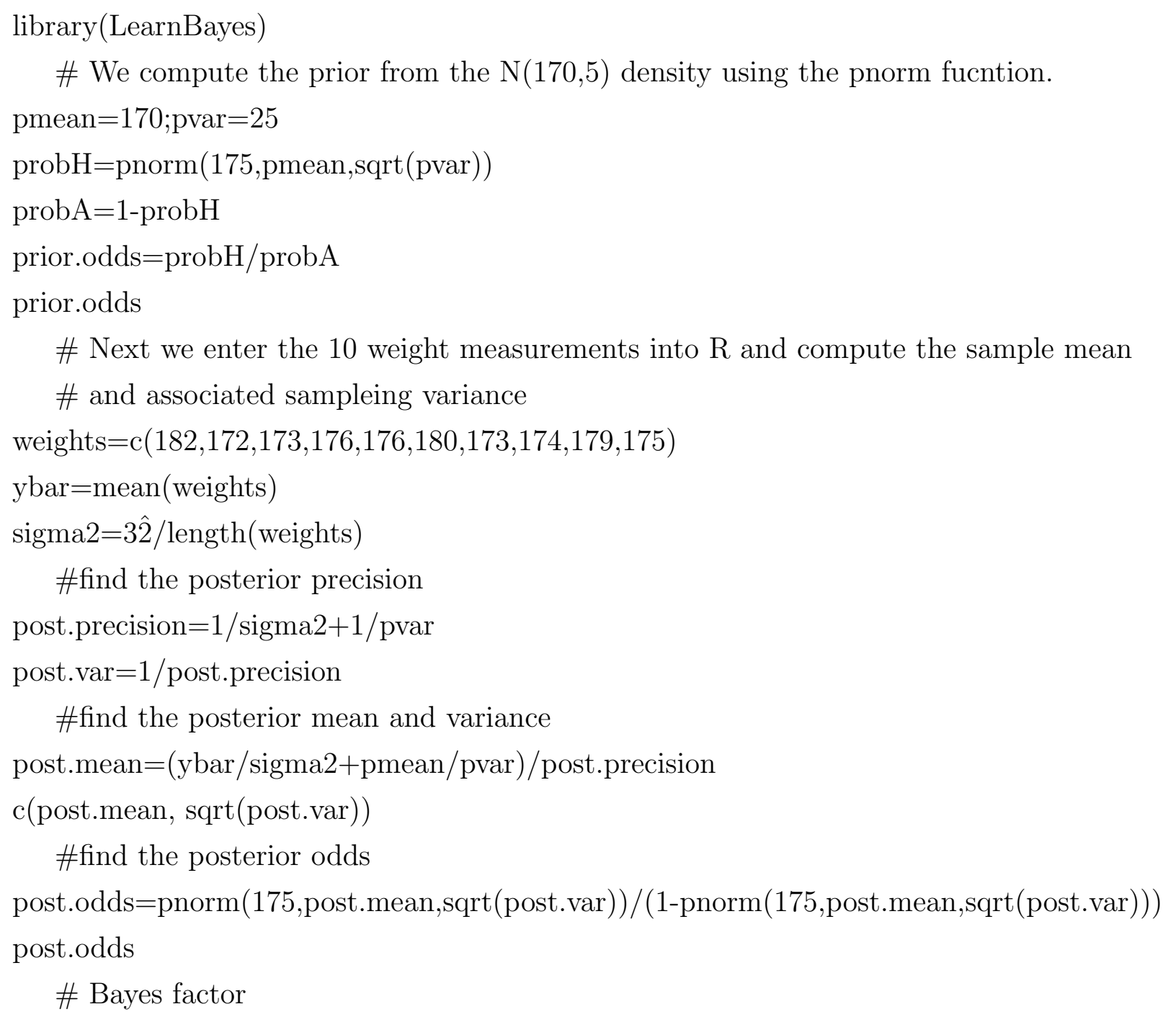




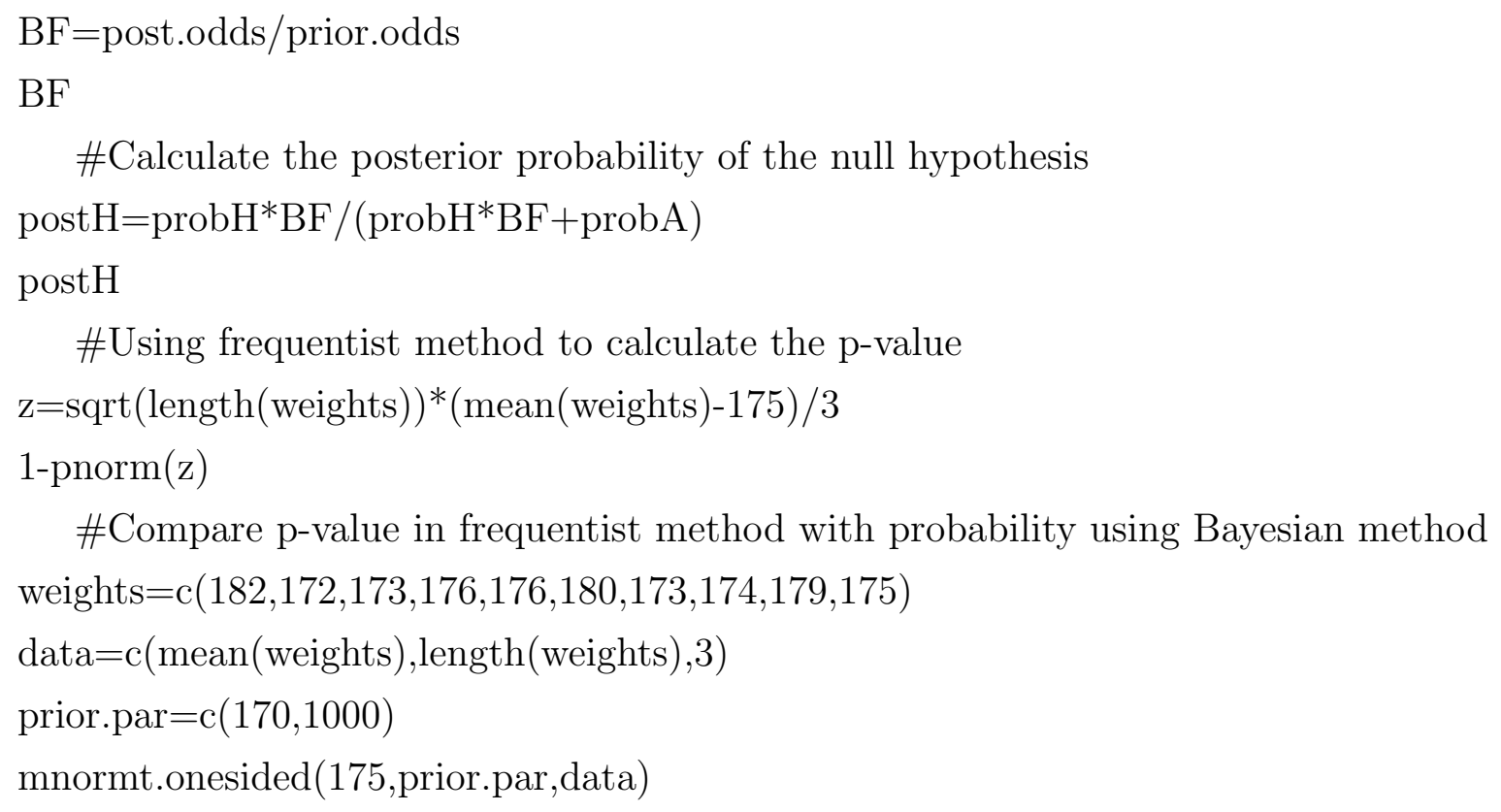

\section{R Program for Simulations 1, 2 and 3}

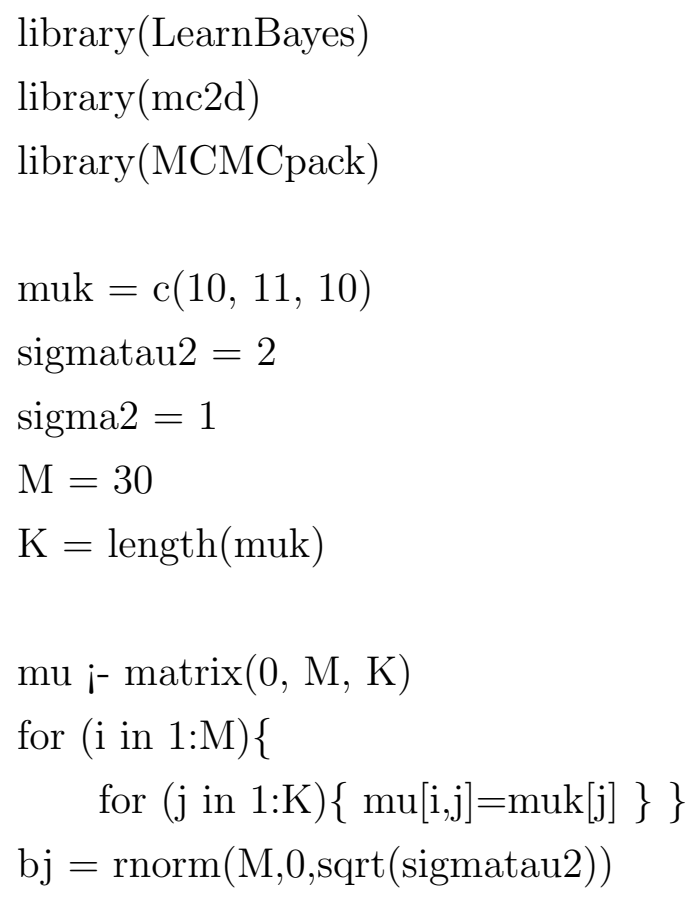




$$
\begin{aligned}
& \mathrm{z}=\operatorname{matrix}(1, \mathrm{M}, \mathrm{K}) \\
& \text { error }=\operatorname{matrix}\left(\operatorname{rnorm}\left(\mathrm{K}^{*} \mathrm{M}, 0, \operatorname{sqrt}(\operatorname{sigma} 2)\right), \mathrm{M}, \mathrm{K}\right) \\
& \mathrm{y}=\operatorname{matrix}(0, \mathrm{M}, \mathrm{K}) \# \text { define a matrix }\left(\mathrm{km}^{*} 1\right) \text { for } \mathrm{y} \\
& \mathrm{y}=\mathrm{mu}+\mathrm{z}^{*} \mathrm{bj}+\text { error ydata }=\mathrm{y} \\
& \text { iteration }=10000 \\
& \mathrm{a} 0=2.1 ; \mathrm{b} 0=0.001 \\
& \mathrm{ar}=230 ; \mathrm{br}=60 \\
& \mathrm{mu} 0=0 ; \operatorname{tao} 02=10000
\end{aligned}
$$

mu1ini $=10 ;$ sigma2ini $=1 ;$ sigmatau2ini $=2 ;$ delta0ini $=\mathrm{c}(0,0,0,0)$

$\mathrm{pr}=0.5 ; \mathrm{sigmaD} 2=0 ; \mathrm{ai} 0=0 ; \mathrm{ai} 1=0 ; \mathrm{gi}=0 ; \mathrm{h} 0=0 ; \mathrm{h} 1=0 ;$ lamda $=0$

mu1store $=\operatorname{matrix}(0,2$, iteration $) ; \operatorname{mu} 1$ store $[, 1]=\operatorname{mu} 1$ ini sigma2store $=\operatorname{matrix}(0,2$, iteration $) ;$

sigma2store[,1]= sigma2ini sigmatau2store $=\operatorname{matrix}(0,2$, iteration $) ;$ sigmatau2store $[, 1]=$ sigmatau2ini

delta0store $=\operatorname{matrix}(0, \mathrm{~K}-1+2$, iteration $)$ for $(\mathrm{j}$ in $1:(\mathrm{K}-1+2))\{\operatorname{delta0} 0$ store $[\mathrm{j}, 1]=\operatorname{delta0} 0 \mathrm{ini}[\mathrm{j}]$ \}

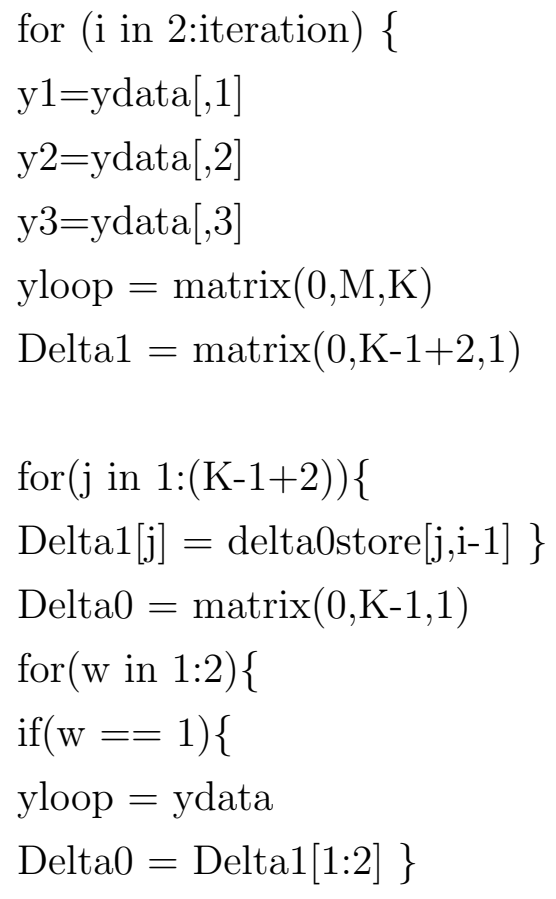




$$
\begin{aligned}
& \text { else\{ } \\
& \text { yloop[,1] }=\text { y2 } \\
& \text { yloop }[, 2]=y 1 \\
& \text { yloop }[, 3]=\text { y3 } \\
& \text { Delta0 }=\operatorname{Delta1}[3: 4]\} \\
& \text { Mulloop }=\text { mu1store }[\mathrm{w}, \mathrm{i}-1] \\
& \text { Sigma2loop }=\text { sigma2store }[\mathrm{w}, \mathrm{i}-1] \\
& \text { Sigmatau2loop }=\text { sigmatau2store }[\mathrm{w}, \mathrm{i}-1] \\
& \mathrm{Mu} 1=0 \\
& \text { Sigma2 }=0 \\
& \text { Sigmatau } 2=0 \\
& \mathrm{~S} 1=0 \\
& \mathrm{~S} 2=0 \\
& \mathrm{~S} 3=0 \\
& \mathrm{~S} 4=0 \\
& \operatorname{diff}=0 \\
& \operatorname{diffS} 2=0 \\
& \operatorname{diffS} 4=0 \\
& \operatorname{diffS} 41=0 \\
& \text { testdelta }=\mathrm{c}(0, \text { Delta } 0) \\
& \text { for }(\mathrm{r} \text { in } 1: \mathrm{M})\{ \\
& \text { for(s in } 1: K)\{ \\
& \text { diff }=\text { diff }+ \text { testdelta }[\mathrm{s}] \text { \# value for first sumation of delta } \\
& \mathrm{S} 1=\mathrm{S} 1+(\text { yloop}[\mathrm{r}, \mathrm{s}]-\text { Mu1loop-diff })^{\wedge} 2 \text { \# value for factor S1 } \\
& \mathrm{S} 3=\mathrm{S} 3+(\mathrm{yloop}[\mathrm{r}, \mathrm{s}] \text {-diff }) \text { \# value for factor S3 } \\
& \text { diffS2 }=\text { diffS2 }+(\text { yloop}[\mathrm{r}, \mathrm{s}] \text {-Mu1loop-diff })\} \\
& \mathrm{S} 2=\mathrm{S} 2+\operatorname{diffS} 2^{\wedge} 2 \\
& \operatorname{diff}=0 \operatorname{diffS} 2=0\}
\end{aligned}
$$

\#\#\#\#\# Estimate Parameter Sigma2

$$
\begin{aligned}
& \mathrm{p} 2=\mathrm{M}^{*}(\mathrm{~K}-1) / 2 \\
& \mathrm{q} 2=(\mathrm{S} 1-\mathrm{S} 2 / \mathrm{K}) / 2
\end{aligned}
$$


Sigma2 $=(\mathrm{q} 2)^{*}$ rinvgamma $(1, \mathrm{p} 2) ; \operatorname{sigma} 2$ store $[\mathrm{w}, \mathrm{i}]=$ Sigma2

\#\#\#\# Estimate Parameter Sigmatau2

Tao2 $=(\mathrm{S} 2 /(2 * \mathrm{~K}))^{*}$ rinvgamma $(1, \mathrm{M} / 2)$

Sigmatau2 $=($ Tao2 - Sigma2) $/$ K sigmatau2store $[\mathrm{w}, \mathrm{i}]=$ Sigmatau2

\#\#\#\# Estimate Parameter Mu1

$$
\begin{aligned}
& \mathrm{p} 1=\mathrm{mu} 0 /\left(2^{*} \operatorname{tao} 02\right)+\mathrm{S} 3 /\left(\mathrm{K}^{*} \operatorname{Sigmatau} 2+\operatorname{Sigma} 2\right) \\
& \mathrm{q} 1=1 / \operatorname{tao} 02+\mathrm{M}^{*} \mathrm{~K} /\left(\mathrm{K}^{*} \operatorname{Sigmatau} 2+\operatorname{Sigma} 2\right) \\
& \mathrm{Mu} 1=\operatorname{rnorm}(1, \mathrm{p} 1 / \mathrm{q} 1, \operatorname{sqrt}(1 / \mathrm{q} 1)) ; \operatorname{mu} 1 \text { store }[\mathrm{w}, \mathrm{i}]=\mathrm{Mu} 1
\end{aligned}
$$

\#\#\#\#\# In order to simulate all the deltai values, we need to calculate the lamda value for the \# Bernoulli distribution first, then if $\mathrm{Br}=1$, set delta $=0$; \# if $\mathrm{Br}=0$, set delta Normal distribution(Gi/Ai,1/Ai)

$$
\begin{aligned}
& \text { for }(\mathrm{n} \text { in } 1:(\mathrm{K}-1))\{ \\
& \mathrm{Pr}=0 \\
& \text { SigmaD2 }=0 \\
& \mathrm{Gi}=0 \\
& \mathrm{Ai} 0=0 \\
& \mathrm{Ai} 1=0 \\
& \mathrm{H} 0=0 \\
& \mathrm{H} 1=0 \\
& \text { Lamda }=0 \\
& \text { Br }=0 \\
& \text { Delta }=0 \\
& \text { S4 }=0 \\
& \text { firstsum }=0 \\
& \text { for }(\mathrm{r} \text { in } 1: \mathrm{M})\{ \\
& \text { secondsum }=0 \\
& \text { for }(\mathrm{t} \text { in }(\mathrm{n}+1): \mathrm{K})\{ \\
& \text { thirdsum }=0 \\
& \text { for }(\mathrm{j} \text { in } 1:(\mathrm{t}-1))\{\text { thirdsum }=\text { thirdsum }+ \text { Delta0[j] }\} \\
& \text { secondsum }=\text { secondsum }+(\mathrm{yloop}[\mathrm{r}, \mathrm{t}]-\mathrm{Mu} 1-\text { thirdsum }+ \text { Delta0 }[\mathrm{n}])\}
\end{aligned}
$$




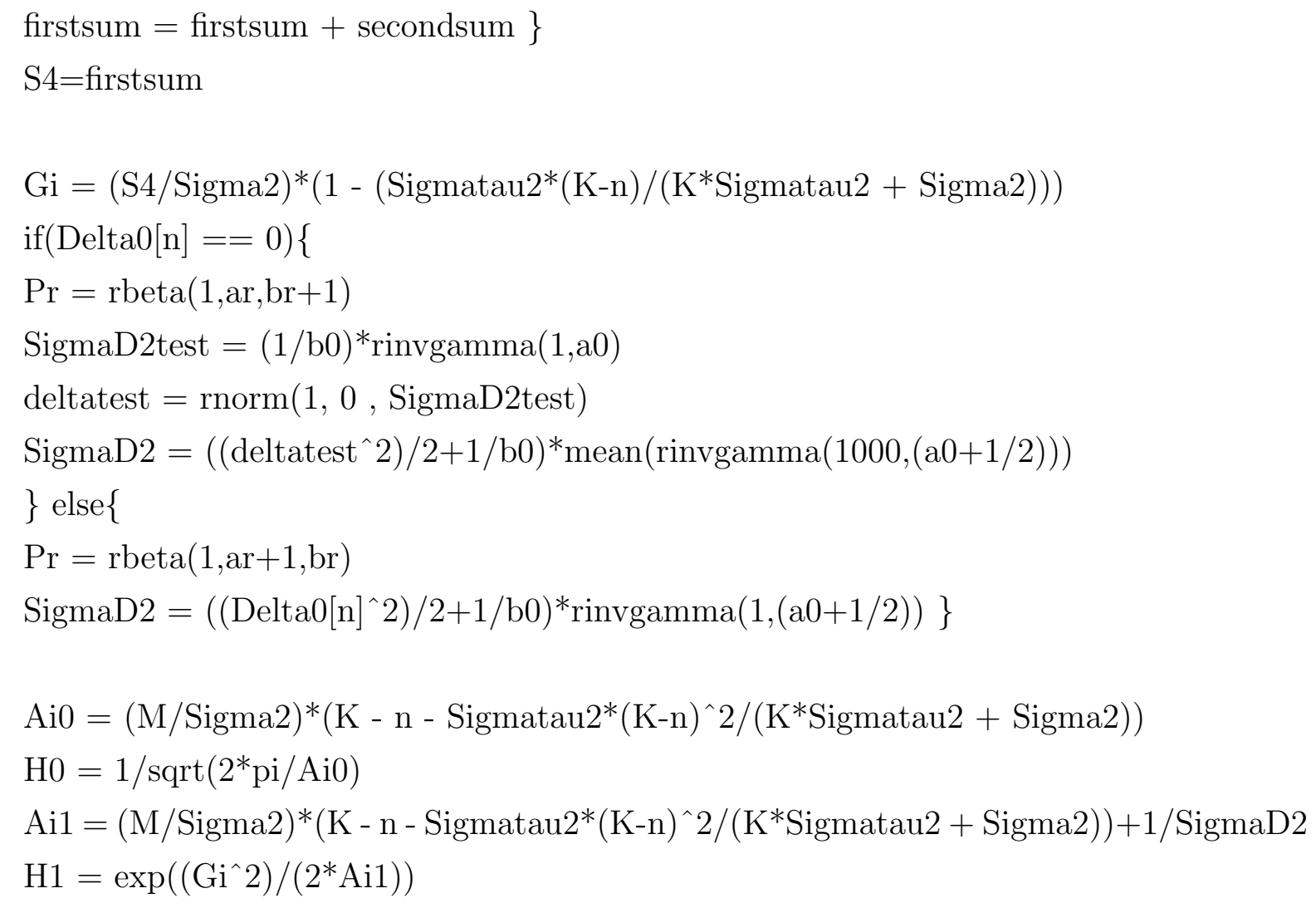

\#\#\#\# After calyculate H0 and H1, then we can use the FDR method to calculate the ratio of Bernoulli distribution and use Bernoulli distr to give true/false

Lamda $=((1-\mathrm{Pr}) * \mathrm{H} 0) /\left((1-\mathrm{Pr}) * \mathrm{H} 0+\mathrm{Pr}^{*} \mathrm{H} 1 / \operatorname{sqrt}(\operatorname{SigmaD} 2)\right)$

$\mathrm{Br}=\operatorname{rbern}(1$, Lamda $)$

if $(\mathrm{Br}==0)\left\{\right.$ Delta $=\operatorname{rnorm}(1, \mathrm{Gi} / \mathrm{Ai1}, \operatorname{sqrt}(1 / \mathrm{Ai} 1)) ; \operatorname{delta0} \operatorname{store}\left[\mathrm{n}+2^{*}(\mathrm{w}-1), \mathrm{i}\right]=$ Delta $\}$

else $\left\{\right.$ Delta $=0 ;$ delta0store $\left[\mathrm{n}+2^{*}(\mathrm{w}-1), \mathrm{i}\right]=$ Delta $\left.\left.\}\right\}\right\}$

\#\#\#\# Checking Final Result

store $=\operatorname{matrix}(\mathrm{c}($ delta0store[1,],delta0store[2,],delta0store[4,]), nrow $=3, \mathrm{ncol}=$ iteration, byrow $=$ TRUE, dimnames=list (c("Delta1", "Delta2", "Delta3"),c(1:iteration $)))$

result $=$ store[,burn:iteration]

count $=$ matrix $(0, \mathrm{~K}, 1)$

count $[1] / 7000$

count[2]/7000

count[3]/7000 


\section{R Program for Simulation 4}

\# In order to find the $\operatorname{Pr}\{\mathrm{Ho}-\mathrm{Y}\}$ for all 5000 datasets, set

numberofdatasets $=5000$

\#\# For average Probablity

PrstoreDeltal $=$ matrix $(0,1$, numberofdatasets $)$

PrstoreDelta $2=$ matrix $(0,1$, numberofdatasets $)$

PrstoreDelta $3=$ matrix $(0,1$, numberofdatasets $)$

PrstoreGloba $=\operatorname{matrix}(0,1$, numberofdatasets $)$

PrstoreJoint $=$ matrix $(0,1$, numberofdatasets $)$

\#\# For Pr\{reject\}, which pro tha less than 0.5

countDelta $1=$ matrix $(0,1$, numberofdatasets $)$

countDelta $2=$ matrix $(0,1$, numberofdatasets $)$

countDelta $3=$ matrix $(0,1$, numberofdatasets $)$

countGloba $=$ matrix $(0,1$, numberofdatasets $)$

countJoint $=$ matrix $(0,1$, numberofdatasets $)$

\#\#\#\# now start the big loop for 3 dataset

for(d in 1:numberofdatasets) \{

\section{Repeat Complete R Code in Simulation 3}

PrstoreDelta1 $[\mathrm{d}]=$ count $[1] /($ iteration-burn $)$

PrstoreDelta2 $[\mathrm{d}]=$ count $[2] /($ iteration-burn $)$

PrstoreDelta3 $[\mathrm{d}]=$ count $[3] /$ (iteration-burn)

PrstoreGloba $[\mathrm{d}]=$ PrstoreDelta1 $[\mathrm{d}]{ }^{*}$ PrstoreDelta2 $[\mathrm{d}]{ }^{*}$ PrstoreDelta3 $[\mathrm{d}]$

PrstoreJoint $[\mathrm{d}]=$ jointcount/(iteration-burn)

if $($ PrstoreDelta1 $[\mathrm{d}]<0.5)\{\operatorname{countDelta1}[\mathrm{d}]=\operatorname{countDelta1}[\mathrm{d}]+1\}$

if $($ PrstoreDelta2 $[\mathrm{d}]<0.5)\{$ countDelta2 $[\mathrm{d}]=\operatorname{countDelta} 2[\mathrm{~d}]+1\}$

if $($ PrstoreDelta3 $[\mathrm{d}]<0.5)\{\operatorname{countDelta} 3[\mathrm{~d}]=\operatorname{countDelta} 3[\mathrm{~d}]+1\}$ 


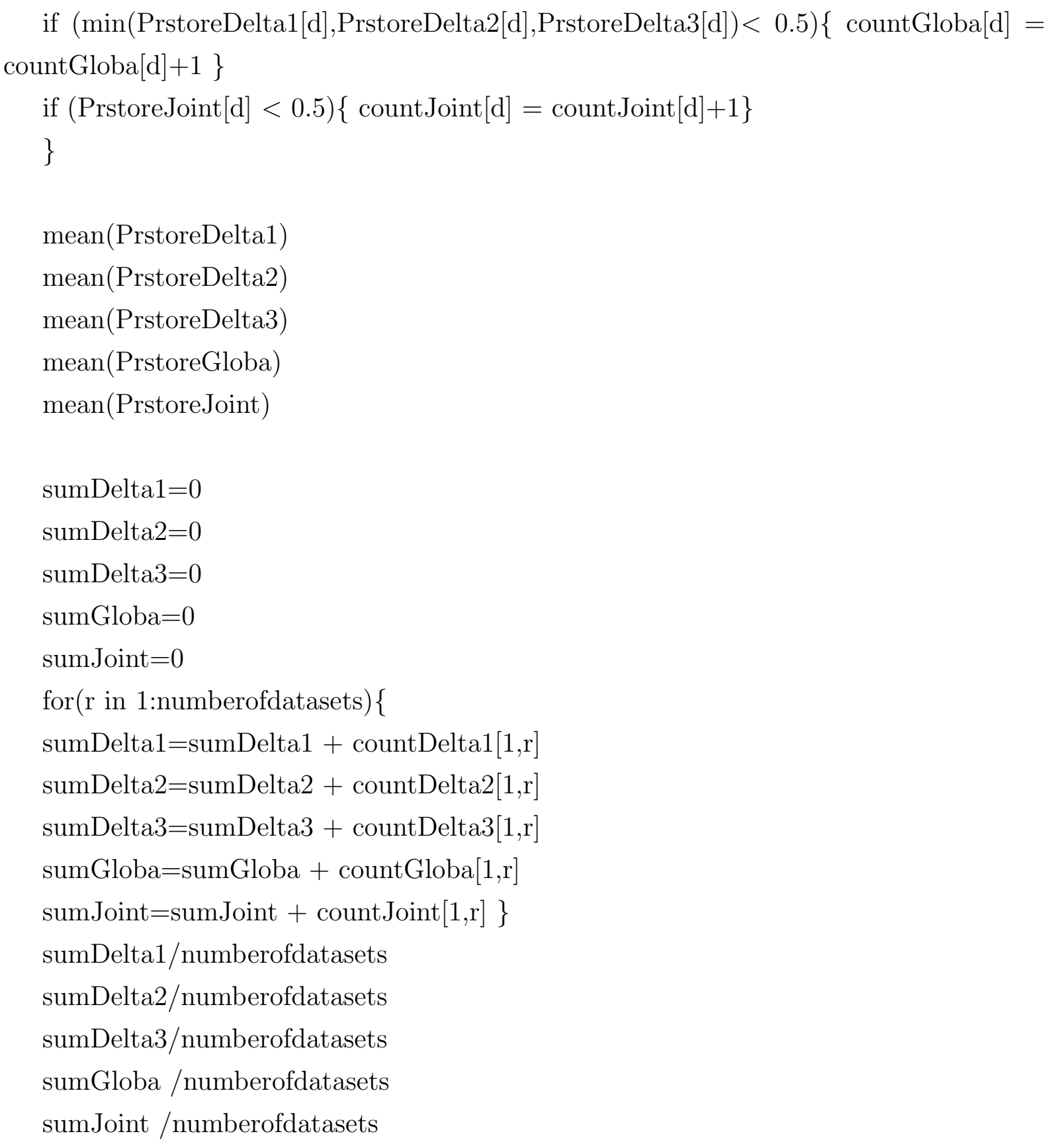

